\title{
Simulation of Cracks in a Cosserat Medium using the eXtended Finite Element Method
}

\author{
by \\ Maria Kapiturova \\ A thesis \\ presented to the University of Waterloo \\ in fulfillment of the \\ thesis requirement for the degree of \\ Master of Applied Science \\ in \\ Civil Engineering
}

Waterloo, Ontario, Canada, 2013

(C) Maria Kapiturova 2013 


\section{Author's Declaration}

I hereby declare that I am the sole author of this thesis. This is a true copy of the thesis, including any required final revisions, as accepted by my examiners.

I understand that my thesis may be made electronically available to the public. 


\begin{abstract}
This research investigates fracture behaviour in the Cosserat materials. The Cosserat elasticity description of the materials incorporates a characteristic length scale (e.g. grains, particles, fibres, etc.) into the model. The characteristic length scale in such materials is known to significantly influence the macroscopic behaviour of the whole body. Simulation of the fracture processes, such as crack opening and propagation, in Cosserat materials still remains a challenge for the scientific community. The goal of this thesis is to propose and validate a two dimensional extended finite element method model of edge cracks within the Cosserat elasticity theory framework.

The crack modelling was conducted using the Finite Element Method (FEM) and eXtended Finite Element Method (XFEM) implemented in the Matlab code. The strong and weak formulations of the problem and the discrete XFEM equations are presented in the thesis. Mode I and II edge crack models in a Cosserat medium are discussed and verified through a series of patch and convergence tests. In addition, the numerical evaluation of the J-integral for the Cosserat medium is presented, and the J-integral for the Cosserat medium is compared to the J-integral for the classical elasticity.

The XFEM/Cosserat method is shown to be robust and able to effectively model the edge crack problems in a Cosserat medium. Moreover, the elastic parameter $\alpha$ is found to be a powerful coupling tool between the microrotations and the translations. The Cosserat J-integral differs from the classical J-integral by $2 \%$ to $40 \%$, for a given crack depending on the micropolar elastic coupling constant $\alpha$.
\end{abstract}




\section{Acknowledgements}

I would like to thank my supervisors, Dr. Robert Gracie and Dr. Stanislav Potapenko, for their guidance and support during my study at the University of Waterloo.

I would like to thank my mom, my grand mom, my brother and his family for their enormous love, care and encouragement.

I would like to thank my dear friends for being supportive and providing warm environment during these two years. 


\section{Dedication}

This thesis is dedicated to my parents, Roman Kapiturov and Svetlana Kapiturova. 


\section{Table of Contents}

List of Tables $\quad$ ix

List of Figures $\quad$ x

1 Introduction $\quad 1$

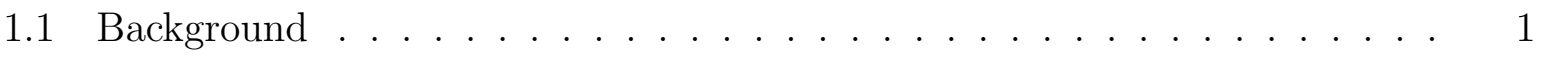

1.2 Scope and objectives . . . . . . . . . . . . . . . . . . 2

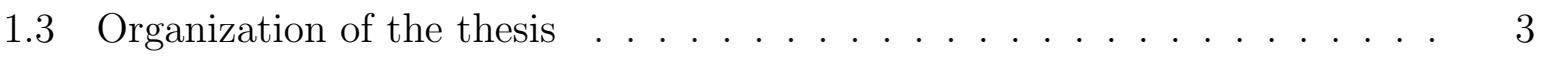

2 Literature Review $\quad 5$

2.1 Cosserat continuum . . . . . . . . . . . . . . . 5

2.1.1 Cosserat materials . . . . . . . . . . . . . 5

2.1.2 Development of the Cosserat theory of elasticity . . . . . . . . . 9

2.1.3 Description of the Cosserat model . . . . . . . . . . . . . . . 12

2.2 Modelling of discontinuities . . . . . . . . . . . . . . . . 16

2.2.1 Existing models of discontinuities . . . . . . . . . . . 16

2.2.2 Methods of numerical modelling of cracks . . . . . . . . . . . 18

2.3 Linear elastic fracture mechanics . . . . . . . . . . . . . . . 20 
2.3.1 Fracture mechanics in the classical theory of elasticity . . . . . . . . 20

2.3.2 Fracture mechanics in the Cosserat theory of elasticity . . . . . . . 24

2.4 Cosserat models coupled with the Finite Element Method . . . . . . . . . . 28

2.5 Cosserat models coupled with the eXtended Finite Element Method . . . . 30

3 Overview of the FEM $\quad 32$

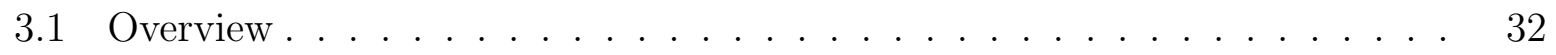

3.2 Shape functions construction . . . . . . . . . . . . . . . 33

3.3 Gauss quadrature . . . . . . . . . . . . . . . . . 37

3.4 Convergence of FEM . . . . . . . . . . . . . . . . . . . . . . . 38

3.4.1 Errors in the approximation . . . . . . . . . . . . 38

3.4.2 Measures of the errors . . . . . . . . . . . . . . . . 38

3.4.3 Accuracy of the solution . . . . . . . . . . . . . . . . 39

3.5 Verification of FEM . . . . . . . . . . . . . . . . . . . . 40

3.5.1 Computation scheme for the error evaluation of the XFEM solution with the FEM exact solution . . . . . . . . . . . . . . . 41

4 XFEM Model of the Crack in the Cosserat Elastic Material 43

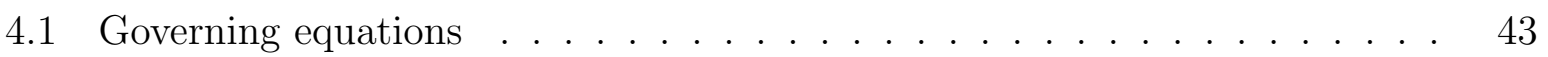

4.2 Weak form ........................ . . . 46

4.3 Discrete equations . . . . . . . . . . . . . . . . . . 46

4.4 Numerical integration . . . . . . . . . . . . . . . . . . . . . 52

4.5 Crack opening model . . . . . . . . . . . . . . . . . . 56

4.6 Computation scheme of the J-integral evaluation . . . . . . . . . . . . . 57 
5 Numerical Examples $\quad 58$

5.1 Introduction . . . . . . . . . . . . . . . . . . . . 58

5.2 Convergence study of the FEM/Cosserat solution for the domain without a crack with $\alpha \neq 0 \ldots \ldots \ldots$. . . . . . . . . . . . . . . . 59

5.3 Convergence study of the XFEM/Cosserat solution of the edge crack problem in the domain with the prescribed analytical solution on the boundaries with $\alpha=0 \ldots \ldots \ldots \ldots$. . . . . . . . . . . . . . . . . . . . . .

5.4 Convergence study of the XFEM/Cosserat solution of the edge crack prob-

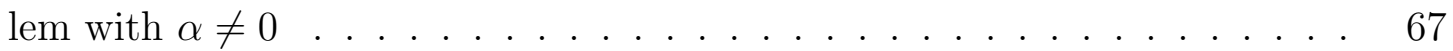

5.4 .1 Mode I edge crack . . . . . . . . . . . . . . . . 67

5.4 .2 Mode II edge crack . . . . . . . . . . . . . . . . . . 70

5.5 Calculation of the J-integral in a Cosserat medium . . . . . . . . . . . 72

5.5.1 Verification of the J-integral calculation . . . . . . . . . . . . . 72

5.5.2 Comparison of the J-integral for the Cosserat elastic medium and classical elastic medium . . . . . . . . . . . . . . . . . . 76

6 Conclusions and Recommendations $\quad 80$

6.1 Concluding remarks . . . . . . . . . . . . . . . . . . . 80

6.2 Recommendations for the future development . . . . . . . . . . . . . 81

$\begin{array}{lc}\text { APPENDICES } & 83\end{array}$

$\begin{array}{lr}\text { A Matrix definitions } & 84\end{array}$

$\begin{array}{lr}\text { References } & 90\end{array}$ 


\section{List of Tables}

3.1 Nodal coordinates in the parametric element domain. . . . . . . . . . . 36 


\section{List of Figures}

2.1 Cosserat materials: (a) layered rock (ipb.ac.rs), (b) rock mass (qusterre.com), (c) porous concrete $($ soa.utexas.edu) . . . . . . . . . . . 7

2.2 Cosserat materials: (a) polymer (sbio.uct.ac.za), (b) composite (fz-juelich.de), (c) cellular solid (web.mit.edu), (d) bone (mhhe.com). . . . . . . . 8

2.3 The representation of the degrees of freedom in the Cosserat model. . . . . 12

2.4 Representation of the force balance in (a) classical elasticity, and (b) Cosserat elasticity. . . . . . . . . . . . . . . . . . 13

2.5 Moment equilibrium in 2D . . . . . . . . . . . . . . 15

2.6 Weak discontinuities: (a) material interface (materialinterface.com), (b) inclusion (skywalker.cochise.edu). . . . . . . . . . . . . . 17

2.7 Strong discontinuities: (a) crack (msnbc.msn.com), (b) dislocation (Loudet

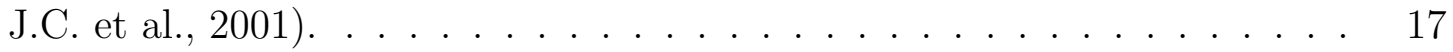

2.8 Representation of the coordinate systems: $\left(x_{1}, x_{2}\right)$ - global coordinates, $\left(\bar{x}_{1}, \bar{x}_{2}\right)$ - local crack coordinates, $(r, \theta)$ - polar coordinates. . . . . . .

2.9 The representation of distribution of $\frac{\tilde{K}_{I}}{K_{I}}$ as a function of $\frac{\alpha}{E}$, parametrized by $\gamma$ (Diegele et al., 2004) . . . . . . . . . . . . . . . 26

2.10 The representation of distribution of $L_{I}$ as a function of $\frac{\alpha}{E}$, parametrized by

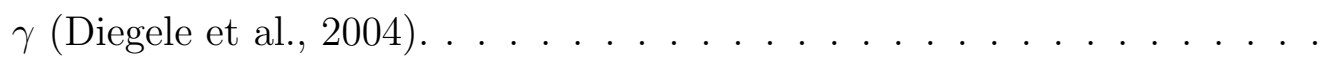

3.1 The representation of the shape functions for the two-node element. . . . . 
3.2 The representation of the shape functions for the rectangular element in $2 \mathrm{D} .35$

3.3 Mapping from the parent coordinates to the physical coordinates (Fish, Belytschko. A First Course in Finite Elements). . . . . . . . . . . . . . 36

4.1 Body with external and internal boundaries subjected to loads. . . . . . . . 44

4.2 Illustration of a two dimensional domain containing a crack $\Gamma_{c r}$. The circled nodes are enriched by the Heaviside function, and the squared nodes are enriched by the crack tip enrichment function. . . . . . . . . . . . . . . 47

4.3 Level set method for the crack growth modelling. . . . . . . . . . . . . . . 48

4.4 The representation of the Gauss quadrature rule in the element containing crack tip. . . . . . . . . . . . . . . . . . 53

4.5 Selected elements for the geometrical enrichment with the number of elements: (a) $11 \times 11$, (b) $51 \times 51 \ldots \ldots \ldots$. . . . . . . . . . . . 54

4.6 Selected elements for the topological enrichment with the number of elements: (a) $11 \times 11$, (b) $51 \times 51 \ldots \ldots \ldots$. . . . . . . . . . . . 55

5.1 The square domain without a crack. . . . . . . . . . . . . . . 59

5.2 Convergence rates of the FEM solutions for the $\alpha=0,100,1000$. . . . . . 60

5.3 Edge crack in a finite domain. . . . . . . . . . . . . . . . . 61

5.4 Convergence study of the Mode I edge crack problem in a Cosserat medium with $\alpha=0$. . . . . . . . . . . . . . . . . . . .

5.5 Convergence study for the coupled Mode I edge crack case solved by XFEM with topological tip enrichment with varying number of enriched nodes layers from 1 to 4 . . . . . . . . . . . . . . . . . . . . . . . . . . . 65

5.6 Convergence study for the coupled Mode I edge crack case solved by XFEM with geometrical tip enrichment with varying radius from $L / 5$ to $L / 20$. . 66

5.7 The domain with an edge crack under tension. . . . . . . . . . . . . . 67 
5.8 Convergence rates of the XFEM solutions of the edge crack problem under the tension loading with $\alpha=1000 \ldots \ldots \ldots \ldots$

5.9 The square domain with an edge crack under shear loading. . . . . . . . . 70

5.10 Convergence rates of the FEM solutions of the edge crack problem under the shear loading for the $\alpha=1000 \ldots \ldots \ldots \ldots$. . . . . . . . . 71

5.11 The domain with an edge crack. The asymptotic field solutions are prescribed on the boundaries. . . . . . . . . . . . . . . . . 72

5.12 The representation of the J-integral dependence on the contour radius. . . 73

5.13 The representation of dependence of J-integral from $K_{I}$ with $L_{I}=0 . \quad \ldots \quad 75$

5.14 The domain with an edge crack under shear. . . . . . . . . . . . 76

5.15 The J-integral versus the crack length. . . . . . . . . . . . . 78

5.16 The normalized J-integral with respect to the crack length. . . . . . . . . 79 


\section{Chapter 1}

\section{Introduction}

\subsection{Background}

The classical linear theory of elasticity began developing in 1820, based on the original works of Cauchy and Navier [1, 2]. The theory deals with the elastic deformations of solid materials and has numerous applications in engineering. In 1880-1890, Voigt and Duhem made the first attempts to generalize the conventional theory of elasticity by introducing an independent moment vector and polar medium, where the stress tensor is not symmetric. The first complete theory based on these assumptions was presented by the Cosserat brothers in 1909 and was named Cosserat theory of elasticity. Unfortunately, the proposed theory was not widely accepted by the scientific society due to its unclear formulation and generality (the theory considered the problems of fluid mechanics, electrodynamics and magnetism, in addition to the elasticity problems). However, in 1970-1980, with the invention and development of new materials, such as composites, polymers, etc., the Cosserat theory was reconsidered. A large number of experimental and theoretical studies have shown that the behaviour of some materials cannot be described by the classical elasticity explicitly. The studies of Roderic Lakes in 1980s' have had a significant impact on the development and understanding of the Cosserat theory. In his works, he classified human and bovine bones as a Cosserat material, conducted experimental research on the Cosserat elastic parameters, and proved the advantages of the Cosserat theory over the 
classical theory for describing the materials with a complex microstructure. Later, the invention of such powerful technique as the Finite Element Method (FEM) made it possible to conduct the numerical modelling of Cosserat materials behaviour, although mostly in the geomechanical area $[3,4,5,6]$.

The Cosserat model is classified as a generalized continuum model, having additional degrees of freedom (also called higher-order continuum model). Therefore, the model can be used for a research of numerous recently developed materials. For example, Cosserat medium may be considered as a model for granular materials, describing the development of shear bands with the help of rotational degrees of freedom. It can also be considered as a homogenized continuum for discrete and layered materials, and materials with periodic or random microstructures. Also, the Cosserat theory may predict size-effect in bone-like structures and cellular media. There is a big potential for research in the area of modelling materials with complex microstructure behaviour using the Cosserat theory of elasticity.

In this research, the Cosserat theory of elasticity is considered as a new and promising way to describe fracture processes such as crack opening in a Cosserat medium. However, there are a few challenges in the fracture analysis of Cosserat materials due to a lack of studies of fracture parameters such as stress intensity factors and their relations with the J-integral.

\section{$1.2 \quad$ Scope and objectives}

The scope of this thesis is to develop an effective fracture model in a Cosserat elastic medium. The numerical tools used for the research are the Finite Element Method and the eXtended Finite Element Method. Matlab was used as a numerical computing environment to model crack opening problems. The XFEM/Cosserat model was verified by comparing the obtained numerical solution with the known analytical solution and the exact FEM solution, as well as by conducting convergence studies.

The objectives of the thesis are to:

- conduct a literature review to understand the Cosserat elasticity theory, fracture mechanics concepts, and numerical methods used in the research; 
- describe the Cosserat model, the derivation of the strong and weak forms, and the discrete equations for the numerical methods;

- provide examples showing the verification, convergence and effectiveness of the model;

- present the verified calculation of the J-integral for Cosserat solids;

- compare the J-integral for Cosserat elastic solid and classical elastic solid;

- show the dependence of the Cosserat J-integral on the crack sizes; and

- discuss the results of fracture modelling in Cosserat media, giving further recommendation for the development and proposing the possible practical applications of the model.

\subsection{Organization of the thesis}

Chapter 2 provides an introduction and a literature review of the Cosserat elasticity theory and the history of its development. The concepts of Linear Elastic Fracture Mechanics for the classical and Cosserat elasticity theories are described. The types of discontinuities and numerical methods for the modelling are overviewed. The existing papers on the Cosserat problems solved by the Finite Element Method and the eXtended Finite Element Method are discussed.

Chapter 3 gives an overview of the Finite Element Method formulation. The construction of approximations and trial solutions are presented. The error estimation method is discussed.

Chapter 4 describes the eXtended Finite Element Method model of the crack opening problem in a Cosserat medium. The strong and weak forms of the problem are stated. The derivation of the governing equations and the discrete equations are presented. Numerical integration scheme, the algorithm of the crack opening problem, and the J-integral computation scheme are described. 
Chapter 5 presents the numerical modelling of the fracture processes in a Cosserat material: the crack opening problems, the verification and convergence tests, and the discussion of the obtained results. In addition, the numerical evaluation of J-integral is presented.

Chapter 6 constitutes the conclusion of the research project and offers the recommendations for the future development of the topic. 


\section{Chapter 2}

\section{Literature Review}

This section describes the historical perspective and provides an up-to-date overview of the Cosserat theory. The current methods of modelling discontinuities in classical and Cosserat materials are presented.

\subsection{Cosserat continuum}

\subsubsection{Cosserat materials}

A number of modern materials have complex microstructures, which generally involve characteristic length scales. Examples of the characteristic length scales are grains, particles, fibres, cells, etc. [7]. Conventional classical continuum mechanics approaches do not incorporate the effect of the intrinsic length into the models, whereas the description of such materials as a Cosserat continuum takes that effect into account. The Cosserat theory of elasticity was developed in the beginning of the twentieth century and is also known as the micropolar theory of elasticity $[8,9,10]$. Numerous experiments conducted by the researchers in the 1970s and 1980s $[11,12,13]$ showed that the Cosserat theory of elasticity described the mechanical behaviour of the materials with characteristic length scales explicitly, while the classical theory of elasticity was not capable of doing so. Due 
to these observations, the Cosserat theory became especially interesting for the researchers who were developing advanced technologies and materials. For example, these materials are carbon-fibre-reinforced composite materials that are used in aerospace components and in spacecraft construction [14], porous concrete used in civil engineering [15], orthopaedic implants used in biomechanics [16], etc.

The examples of the materials that could be described by the Cosserat theory of elasticity are presented in Figures 2.1 and 2.2. The characteristic microstructure can be easily recognized for each of the shown materials. 


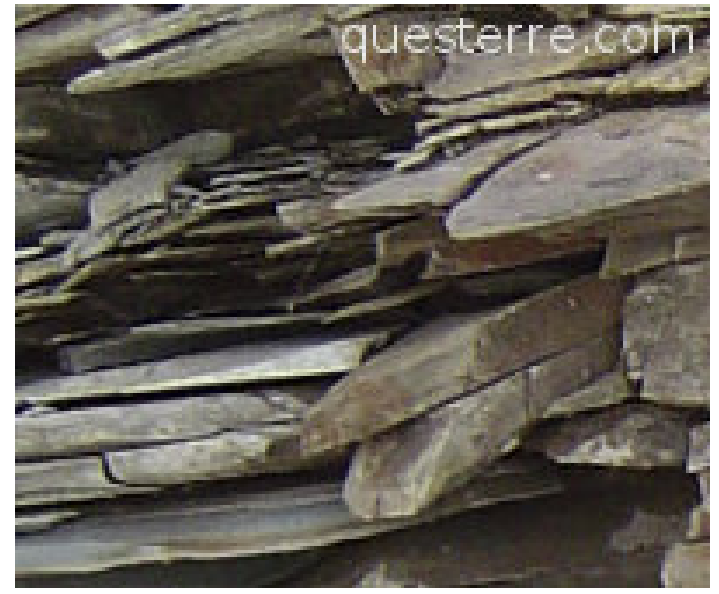

(a)

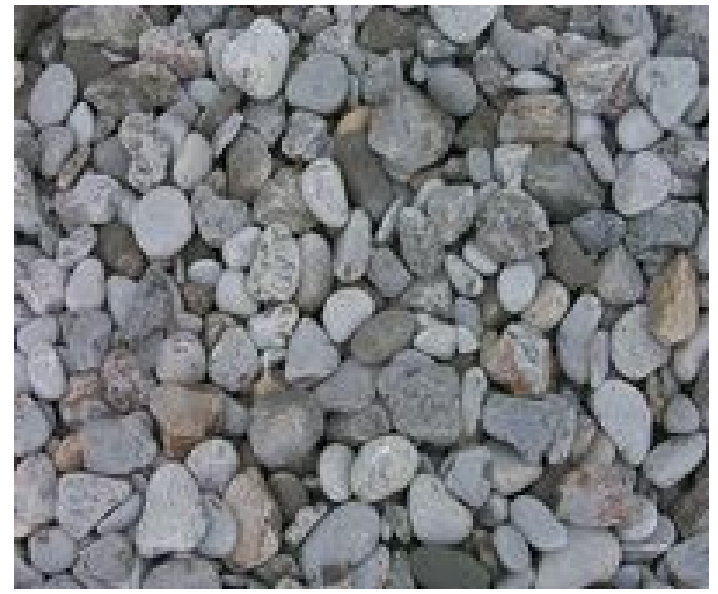

(b)

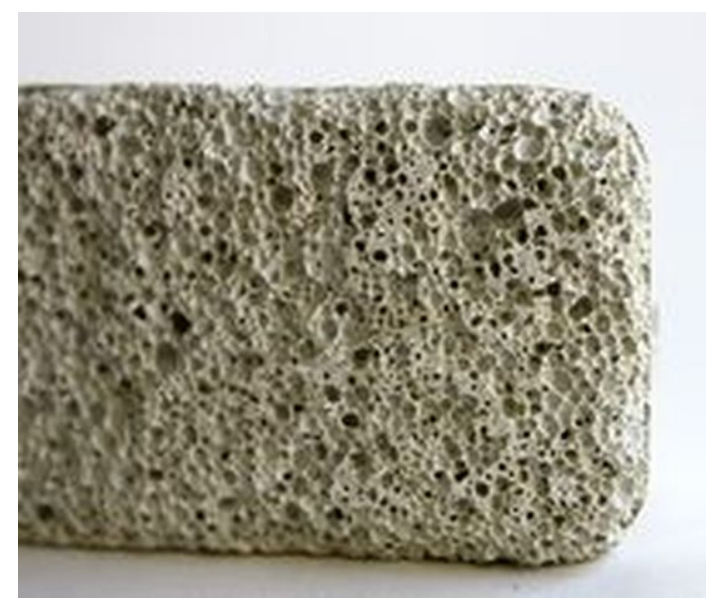

(c)

Figure 2.1: Cosserat materials: (a) layered rock (ipb.ac.rs), (b) rock mass (qusterre.com), (c) porous concrete ( soa.utexas.edu). 


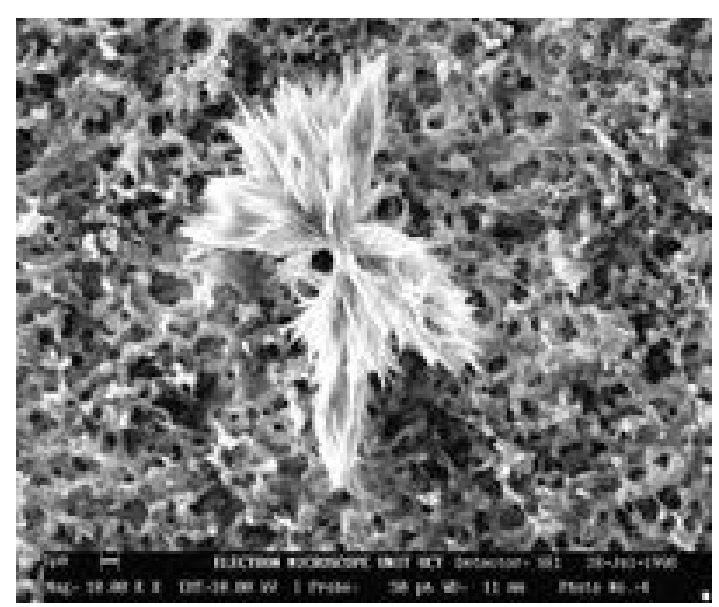

(a)

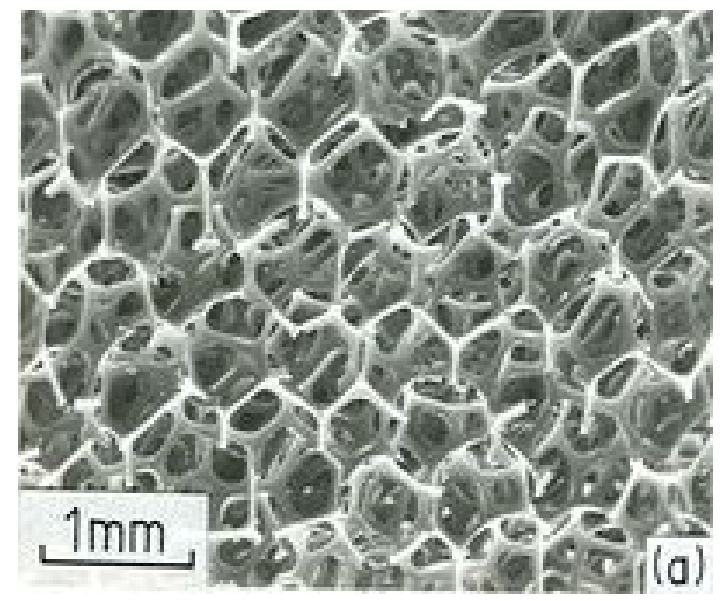

(c)

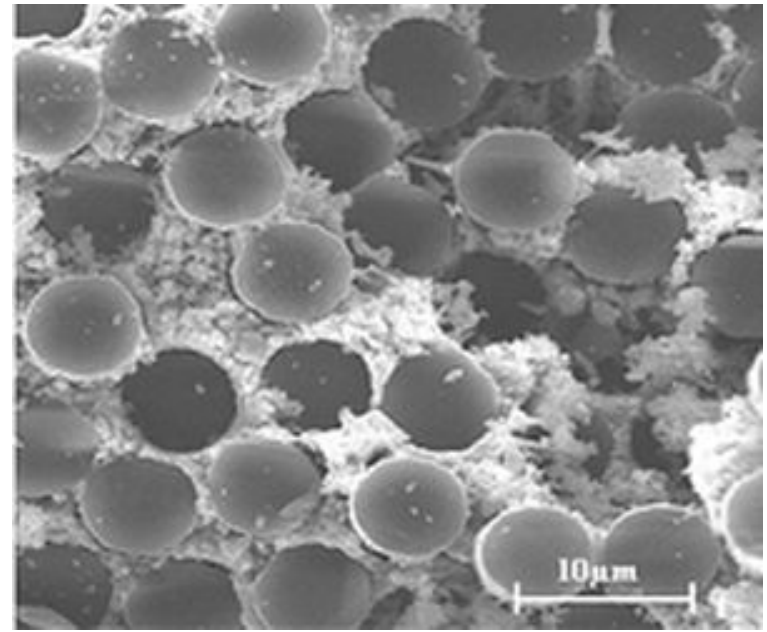

(b)

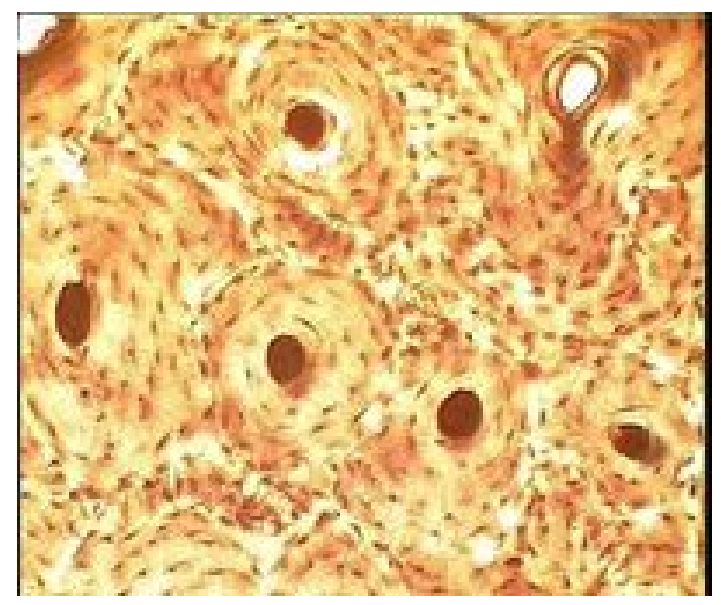

(d)

Figure 2.2: Cosserat materials: (a) polymer (sbio.uct.ac.za), (b) composite (fz-juelich.de), (c) cellular solid (web.mit.edu), (d) bone (mhhe.com). 


\subsubsection{Development of the Cosserat theory of elasticity}

Since the classical theory of elasticity [17] was not able to accurately describe the mechanical behaviour of the materials with complex microstructures, the new extended theory of elasticity began to be considered by scientists. In 1886, Voigt described the medium where an independent moment vector, in addition to a conventional force vector, was introduced to transfer the loading through the surface of the body [18]. According to this assumption, the stress and strain tensors were asymmetric. Then, in 1893, Duhem suggested that the materials could be visualized and described as the sets of points having vectors attached to them, named oriented or polar medium [19].

Later, the first complete extended elasticity theory based on Voigt's assumptions was introduced by the brothers Francois and Eugene Cosserat in 1909 [9]. The authors described the material transformation by translations (displacements) and infinitesimal microrotations at each point of the body. They also derived the equations of balance of forces and balance of angular momentum. The Cosserat brothers created a unified theory to describe solid and fluid mechanics, elastodynamics, and magnetism; however, that theory was too sophisticated for that time. The simplified Cosserat theory with the constrained microrotations was suggested by Truesdell and Toupin in [20, 21], and, later, described by Kupradze in [22]. The name of the developed theory with constrained rotations was the couple stress theory or Cosserat pseudo continuum theory. The rotation vector was dependent on the displacement vector; therefore, the deformation of the body was described solely by the conventional displacement field. However, the stress tensor remained asymmetric so that the equilibrium with the generating couple stresses was preserved. Meanwhile, the research on the Cosserat theory with the independent rotations was conducted by Gunter [23] and Shaefer [24, 25], Aero and Kuvshinsky [26], and Palmov [27].

Eringen further developed the Cosserat theory of elasticity in 1966. He showed that the classical theory of elasticity and the couple stress theory are the special cases of the Cosserat elasticity theory $[8,28]$. At the same time, a full description of the Cosserat theory was presented by Nowacki in [10]. In his works, Nowacki introduced the broad description of the static problems of asymmetric elasticity, problems of thermoelasticity and thermopiezoelectricity in a micropolar medium. The Cosserat medium descriptions of 
Eringen and Nowacki differ insignificantly in terms of Cosserat elastic constants presented in the governing equations and constitutive relations.

According to a number of recent experiments, the Cosserat theory of elasticity usually predicts a size-effect in the materials with characteristic length scales. Size-effect means that smaller samples of the same material behave stiffer than larger samples. The classical theory of elasticity is not capable of explaining this fact. A rigorous study of the size-effect in the Cosserat media was done by $\mathrm{R}$. Lakes et al. in $[29,11,12,30,31]$. In particular, the authors showed that slender cylinders appeared more more stiffly in torsion tests than expected from the classical elasticity. Similar size-effect was also predicted in the bending of plates and of beams, and no size-effects were predicted in tension. Another experiment was presented in [31], where the authors showed that smaller bone specimens considered as a Cosserat elastic continuum had a higher apparent stiffness than thick ones under prolonged torsional load. The Cosserat theory of elasticity accurately fits the experimental data for the effective stiffness of the bone samples from the smallest (osteon) to the whole femur bone [29].

Studies of fracture processes in Cosserat materials were presented by Nakamura, Lakes and other researchers in $[32,33,34,35]$. Lakes showed that the solutions for stress concentration around circular and elliptic holes in plates and stress intensity factors for cracks in Cosserat solid are smaller than the same parameters in a classical solid. This fact is more significant when a crack tip radius is comparable to the dimensions of microstructure and when the size of a hole or a crack is no more than ten times the Cosserat characteristic length.

In the field of today's geomechanics, the Cosserat theory plays an important role. The Cosserat continuum model of jointed rocks was first proposed by Singh in 1973 [36, 37]. After, the further development of the model was presented in [38, 39, 40, 3, 41]. The authors used the Cosserat theory to describe the granular materials under the plane strain condition and shear band formation and its development. A detailed description of the microstructure effect and the scale effect in rock masses is found in [42].

In conclusion, the Cosserat theory of elasticity has a number of advantages over the classical theory of elasticity in describing the behaviour of the materials with characteristic 
length scales. A good summary of general applications of the Cosserat theory is found in $[43,44]$. The Cosserat theory of elasticity may be a replacement for a granular assembly, masonry or blocky structures, where the particle rotations are known to be important in the development of shear bands. In geomechanics, this ability allows to get a large-scale response of the media. The Cosserat theory also allows researchers to homogenize continua for the discrete structural elements and media with periodic and random microstructure. In addition, the Cosserat theory is capable of predicting size-effect in particular mechanical tests (bending and torsion) and of describing the fracture behaviour of the foam-like structures more accurately. 


\subsubsection{Description of the Cosserat model}

The Cosserat theory of elasticity considers a medium as a continuous collection of particles that behave like rigid bodies. The notable characteristics of the theory are

- the independent microrotations of points $\left(\phi_{1}, \phi_{2}, \phi_{3}\right)$ as well as the translations assumed in classical elasticity $\left(u_{1}, u_{2}, u_{3}\right)$ (Figure 2.3);

- the couple stress $\boldsymbol{m}$ (a torque per unit area) as well as the force stress $\boldsymbol{\sigma}$ (force per unit area) (Figure 2.4);

- the four Cosserat elastic constants $(\alpha, \beta, \gamma, \kappa)$, in addition to the two classical Lame constants $(\lambda, \mu)$.

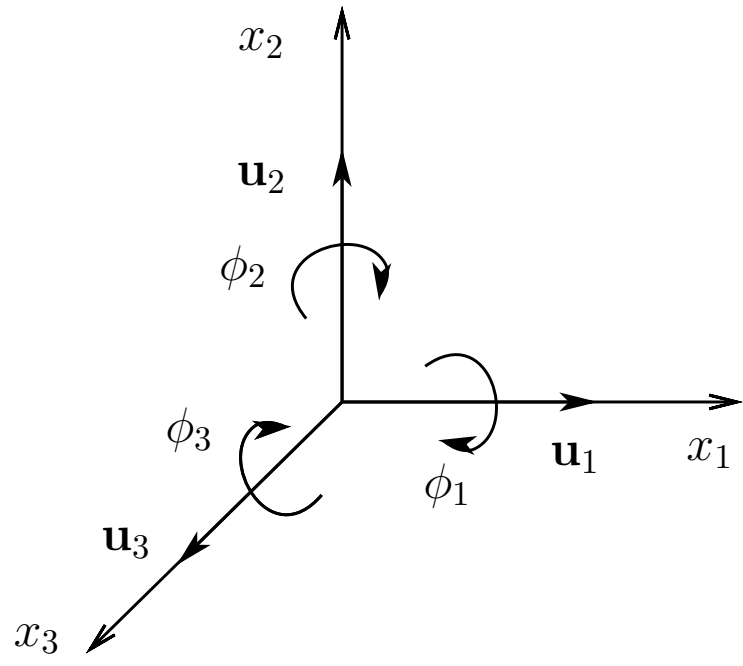

Figure 2.3: The representation of the degrees of freedom in the Cosserat model.

Currently, there are two existing Cosserat models. The first model is the Cosserat model with free rotations, having six degrees of freedom at each point of the material (Figure 2.3 


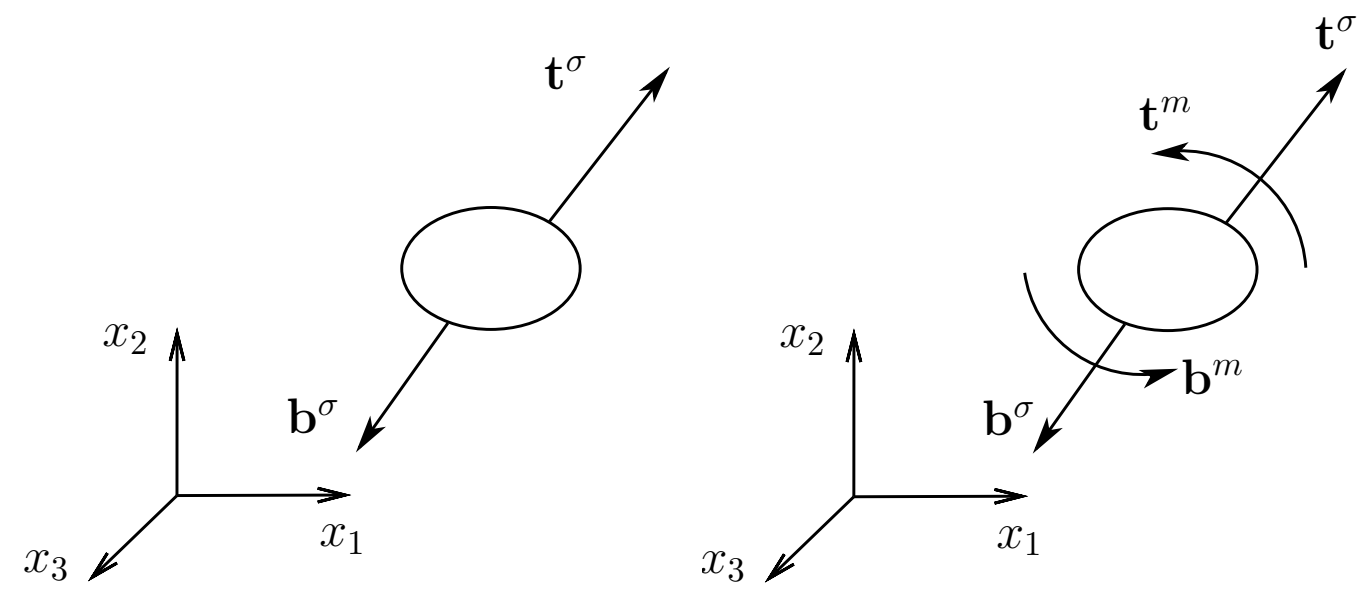

(a)

(b)

Figure 2.4: Representation of the force balance in (a) classical elasticity, and (b) Cosserat elasticity.

[45]). The second model is the Cosserat model with constrained rotations, where particles have only three conventional degrees of freedom (translations). Although the stress tensor is not symmetric, as it is in the first model, the couple stresses are presented to satisfy equilibrium [26]. The Cosserat model with free rotations is described below in detail and is used in this research.

In order for the specific internal energy function to remain positive, the Cosserat material parameters must obey the following inequalities [45]:

$$
\begin{aligned}
& 3 \lambda+2 \mu \geq 0, \quad \mu \geq 0, \quad \alpha \geq 0 \\
& 3 \beta+2 \gamma \geq 0, \quad \gamma \geq 0, \quad \kappa \geq 0 .
\end{aligned}
$$

An important characteristic for Cosserat media is the coupling number $N$, which ranges in value from 0 (classical elasticity theory, corresponds to $\kappa=0$ ) to 1 (indeterminate couple stress theory, corresponds to $\kappa \rightarrow \infty)$ [46]:

$$
N=\left(\frac{\kappa}{2(\mu+\kappa)}\right)^{1 / 2}=\left(\frac{\kappa(1+\nu)}{E+\kappa(1+\nu)}\right)^{1 / 2}
$$


The original technical elasticity constants could be expressed through the Cosserat elastic constants [8, 47]:

$$
\begin{array}{ll}
\text { Young's modulus } & E=\frac{(2 \mu+\kappa)(2 \lambda+2 \mu+\kappa)}{(2 \lambda+2 \mu+\kappa)}, \\
\text { Shear modulus } & G=\frac{(2 \mu+\kappa)}{2}, \\
\text { Poisson's ratio } & \nu=\frac{\lambda}{(2 \lambda+2 \mu+\kappa)} .
\end{array}
$$

Materials with complex microstructures (granular, fibrous, porous, etc.) have a number of intrinsic length scales. In the two-dimensional context, the internal length scale is identified as the characteristic length for bending or torsion that is defined through the material constants [30]. The characteristic length for bending is

$$
l_{b}=\left(\frac{\gamma}{2 \mu+2 \kappa}\right)^{1 / 2}=\left(\frac{\gamma(1+\nu)}{2 E}\right)^{1 / 2}
$$

and for torsion is

$$
l_{t}=\left(\frac{\beta+\gamma}{2 \mu+\kappa}\right)^{1 / 2} .
$$

In the classical solids, the internal characteristic length scale is of the order of the atomic distance; therefore, couple forces do not produce any macroscopic effect. However, in the Cosserat materials, the characteristic length scale is of the order of microns, and couple stresses may influence the mechanical behaviour of the whole body. For instance, the characteristic length scale for steel is $0.05 \mathrm{~mm}$; for bone, $1 \mathrm{~mm}$; and for masonry or rock masses, $100 \mathrm{~mm}[7]$.

One of the essential features of the Cosserat continuum is that the stress tensor is not symmetric, and asymmetric shear stresses are equilibrated by the couple stresses (Figure 2.5). 


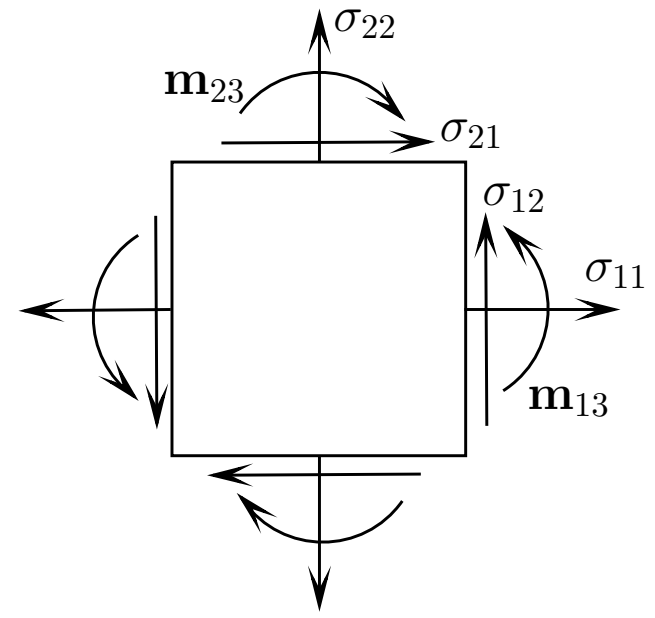

Figure 2.5: Moment equilibrium in 2D. 


\section{$2.2 \quad$ Modelling of discontinuities}

This section presents an overview of the types and models of discontinuities, as well as the description of the numerical methods of modelling.

\subsubsection{Existing models of discontinuities}

There are two main types of discontinuities:

- weak - the displacement field remains continuous, and the strain field may or may not be continuous;

- strong - both the displacement and strain fields are discontinuous.

Weak discontinuities are mainly continuous problems such as material interfaces and inclusions (Figure 2.6), and strong discontinuities are cracks, dislocations, and voids (Figure 2.7).

In the current research, the crack opening in a Cosserat medium is modelled based on the concepts of fracture mechanics and the extended finite element method. In general, crack simulation may be described by the non-local model [48], the continuous smeared crack model [49], the discrete crack model [50], etc.

Non-local models allow researchers to avoid mesh dependency for the simulation of the crack propagation [51]. The model assumes that the fracture behaviour of each point is affected by the stress state at that point and a number of surrounding points. The smeared crack model simulates the mechanics of the crack in terms of stiffness and strength reduction instead of the geometrical modelling of the crack. However, the smeared crack model does not require remeshing while the crack propagates only in the case of the predefined crack path. In the discrete crack model, the discontinuity is defined along the finite element edges or along the sub-mesh inside the elements. Subsequently, the method is expensive and time-consuming because of remeshing. In this research, the discrete crack model is used at one of the stages to simulate crack opening using the Finite Element Method. 


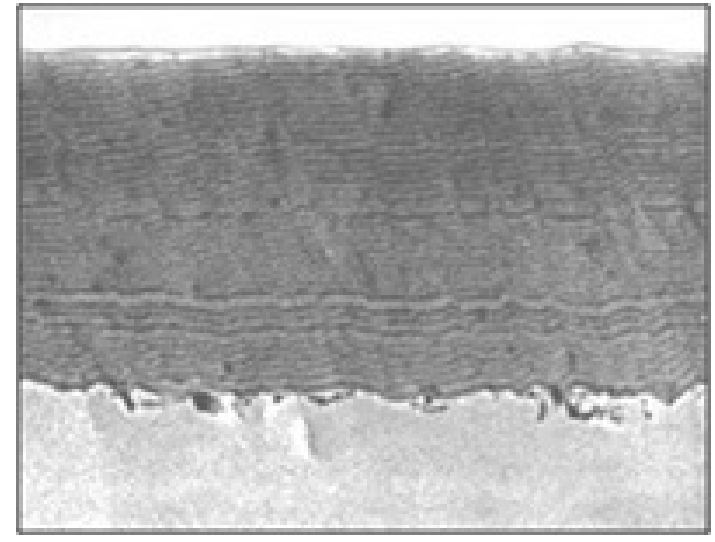

(a)

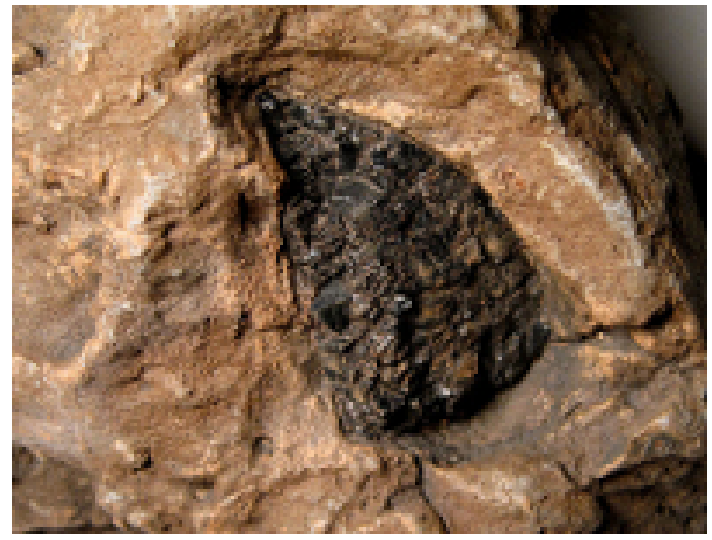

(b)

Figure 2.6: Weak discontinuities: (a) material interface (materialinterface.com), (b) inclusion (skywalker.cochise.edu).

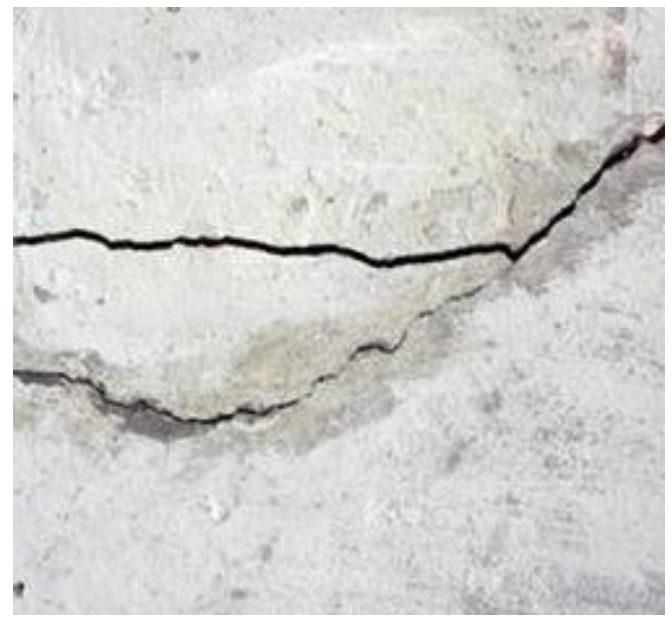

(a)

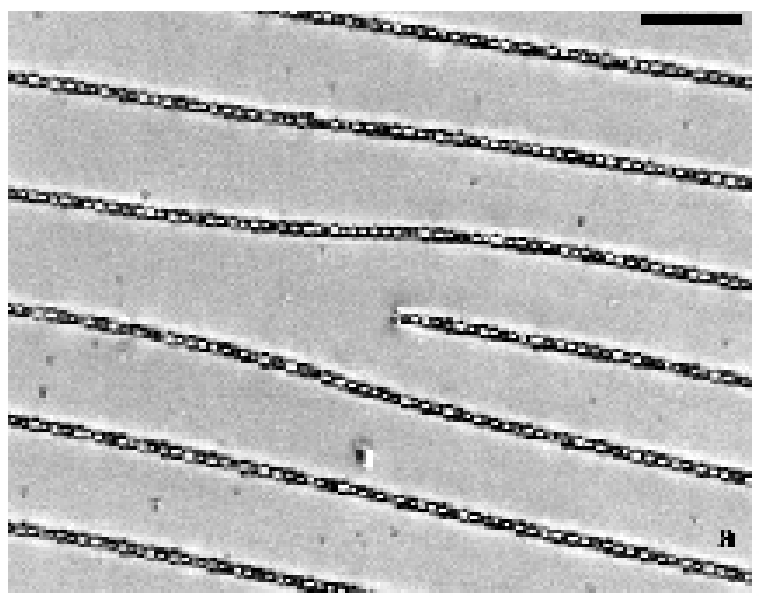

(b)

Figure 2.7: Strong discontinuities: (a) crack (msnbc.msn.com), (b) dislocation (Loudet J.C. et al., 2001). 


\subsubsection{Methods of numerical modelling of cracks}

A number of methods for the simulation of the opening and propagation of cracks exist [51]: analytical, semi-analytical, and numerical approaches that have been developed in the past years. Numerical methods include the boundary integral method [52, 53], the boundary element method [54, 55], the finite element method [56], the extended finite element method [57], the discrete element based method [58], and the meshless method [59].

The basis of the boundary integral method (BIM) is the development of a boundary constraint equation that relates all boundary displacements to all boundary tractions. Both the boundary integral method and the boundary element method (BEM) are restricted to the particular types of problems to which boundary elements can be applied usefully. These are problems with a small surface-volume ratio. However, for many engineering problems, the BEM and BIM are significantly less efficient than the methods such as finite element method. In contrast, the most attractive feature of FEM is its ability to model complicated geometries and boundaries. The finite element method is a powerful numerical tool for the analysis of engineering and physical problems.

The problem of crack modelling in a micropolar medium that is solved in this research is based on the extended finite element method. The method has been already successfully applied for the modelling of different kinds of discontinuities in solids such as cracks [60], interfaces [61], inclusions [62], dislocations [63, 64, 65, 66], etc. The XFEM is a numerical method to model internal (or external) boundaries without requiring the mesh to conform to the boundaries. The technique allows modelling the entire crack geometry independently of the mesh and avoiding the need to re-mesh while the crack propagates. The main idea is to introduce special enrichment functions (for instance, Heaviside function, branch functions, etc.) that depend on the nature of the discontinuity into the standard finite element approximation. Hansbo and Hansbo [67] developed a slightly different method: the phantom node method. This method is based on doubling nodes for every element that is crossed by a discontinuity. The deformation field is interpolated independently on both sides of the discontinuity. The difference between the phantom node method and XFEM lies only in the implementation; however, XFEM is more compatible with the singularity 
enrichments [68].

The extended finite element method was first introduced by Belytschko and Black [57] who added the discontinuous enrichment functions to the test functions and trial solutions. The crack surfaces were considered free of traction. Later Moës et al. in [69] further developed the method and allowed the entire crack to be represented independently from the mesh. Along with the development of XFEM, the Partition of Unity Finite Element Method (PUFEM) and Generalized Finite Element Method (GFEM) were introduced. PUFEM was first presented by Melenk and Babuska in 1996 [70]. Later, Dolbow in his works $[71,72,73]$ combined the jump function and the asymptotic near tip fields solutions to solve two-dimensional elasticity problems.

In 2000, Sukumar et al. in [74] introduced three-dimensional crack modelling. Later, modelling of arbitrary branched and intersecting cracks with multiple branches, kinks, etc., was widely developed. Stolarska et al. [75] introduced the level set method along with XFEM to model crack growth. A level set functions defined the crack location including the location of the tip and were later used to update the location of the cracks [60].

The XFEM approach was applied to a number of the problems of modelling an initialization and propagation of single cracks [76], multiple cracks [77], branching and intersecting cracks [78], curved cracks [79], cohesive cracks [80], etc. XFEM was not only widely presented in the linear crack problems but also had a significant impact on the modelling of contact problems [81, 82], plasticity [83], and large deformation problems [84]. All the aforementioned papers introduce the XFEM method for the modelling crack propagation in classical elasticity media.

Budyn used XFEM to model the growth of multiple cracks in brittle materials [77], presented a multiple scale method for modelling multiple crack growth in cortical bone under tension [85], and developed a method to numerically investigate local stress intensity factors at a scale of osteons in Haversian cortical bone [86]. These works are of special interest because human bones could be represented as a Cosserat medium.

In the area of post-processing the results of the crack modelling problems, for example, the evaluation of fracture mechanics properties, Liu et al. [87] proposed the direct evaluation of the mixed mode stress intensity factors (SIF). They suggested including the higher 
order terms of the crack tip asymptotic field for enriching the finite element approximation of the nodes in the vicinity of the crack tip and applying a penalty function method.

The problem of evaluating the stress intensity factors for thermoelastic cracks was solved in the work of Zamani et al. [88], where the authors used higher order terms of the thermoelastic asymptotic crack tip fields to enrich the approximation space of the temperature and the displacement fields in the vicinity of the crack tip. This paper showed that SIFs were significantly more accurate when they were computed using the interaction integral for both straight and curved cracks.

In conclusion, due to the described advantages of XFEM, it was used as a main numerical tool to model crack opening in the Cosserat material in this research.

\subsection{Linear elastic fracture mechanics}

In this section, the basic concepts of linear elastic fracture mechanics (LEFM) for the classical elasticity and the crack growth criteria are presented, and the fracture parameters for the Cosserat elasticity are described.

\subsubsection{Fracture mechanics in the classical theory of elasticity}

Linear elastic fracture mechanics deals with the materials that are elastic except in a small region around a crack tip. This zone exists due to the fact that the stresses at a crack tip are infinite and that some kind of inelasticity takes place in the immediate vicinity of the crack tip. However, if the size of this zone is small relative to the linear dimensions of the body, the model could be verified exactly [89]. The condition is $L D \geq 8 r_{p}$, where $L D$ is any linear dimension of the problem domain, and $r_{p}$ is the plastic zone size.

There are three types of crack opening modes: normal-opening mode (Mode I), inplane mode (Mode II), and out-of-plane shear mode (Mode III). The current research emphasizes Mode I due to the generality of the model. The opening mode stresses at the region asymptotically close to a crack tip for the classical elastic materials are [51] 


$$
\begin{aligned}
\sigma_{x x} & =\frac{K_{I}}{\sqrt{2 \pi r}} \cos \frac{\theta}{2}\left(1-\sin \frac{\theta}{2} \sin \frac{3 \theta}{2}\right)+\text { h.o.t. } \\
\sigma_{y y} & =\frac{K_{I}}{\sqrt{2 \pi r}} \cos \frac{\theta}{2}\left(1+\sin \frac{\theta}{2} \sin \frac{3 \theta}{2}\right)+\text { h.o.t. } \\
\sigma_{x y} & =\frac{K_{I}}{\sqrt{2 \pi r}} \cos \frac{\theta}{2} \cos \frac{3 \theta}{2} \sin \frac{\theta}{2}+\text { h.o.t., }
\end{aligned}
$$

where $K_{I}$ is a stress intensity factor, $r$ and $\theta$ are the polar coordinates with the origin at a crack tip, and higher order terms are omitted. From equations (2.8), it is clear that, as the crack tip is approaching zero at $r \rightarrow 0$, the presence of term of $\frac{1}{\sqrt{r}}$ leads to singular stresses.

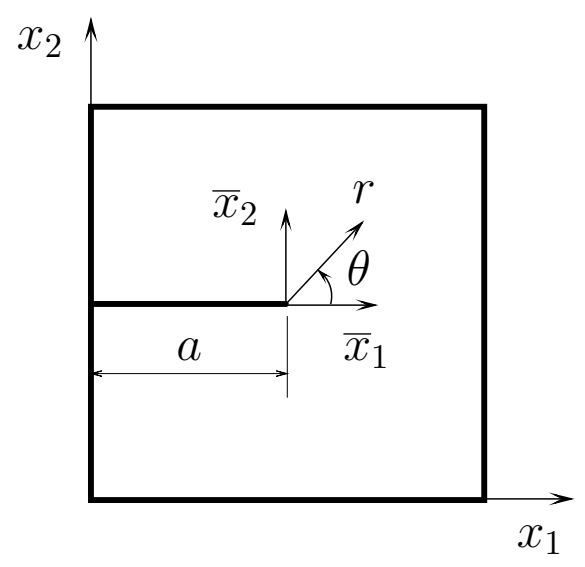

Figure 2.8: Representation of the coordinate systems: $\left(x_{1}, x_{2}\right)$ - global coordinates, $\left(\bar{x}_{1}, \bar{x}_{2}\right)$ - local crack coordinates, $(r, \theta)$ - polar coordinates.

The way a crack propagates through the material indicates the fracture mechanism. 
The most common mechanisms are fatigue fracture and shear fracture. During the fatigue fracture, a crack is subjected to cyclic loading, the crack tip travels a short distance in each loading cycle, and the stress is not high enough to cause a sudden global fracture. Shear fracture is caused by the movement and multiplication of dislocations. This fact leads to the growth of voids and their transfer to the macroscopic cracks. Therefore, a large number of cycles are needed before the total fracture occurs. The loading conditions are important for the crack propagation mechanism as well. They are static, quasi-static, and dynamic. The quasi-static crack growth means that time and inertial mass are irrelevant, i.e., the kinetic energy of the structure is equal to zero, and that the only energy consuming process is fracture. Dynamic fracture means that the energy available exceeds the energy required. Thus, the structure is unstable, and the crack is growing dynamically. In the current research, only the quasi-static process is considered. However, in the possible applications of the method, for example, to the hydraulic fracture, the dynamic crack growth may be modelled.

The development of the brittle fracture hypothesis was initialized by Griffith around 80 years ago [90]. He showed that the product of the far field stress, the square root of the crack length, and the certain material properties control the crack extension in brittle materials. The product was shown to be related to the energy release rate $G$, which represents the elastic energy per unit crack of surface area required for a crack extension. The Griffith fracture criterion approach states that $G=2 \gamma=R$, where $\gamma$ represents the energy required to form the unit of new material surface (the material constant), and $R$ is the crack growth resistance.

Another form of expressing the energy release rate is the J-integral, developed by Cherepanov in 1967 [91] and independently by Rice in 1968 [92]. The path-independent J-integral is a way to calculate the energy per unit fracture surface area. The J-integral vector is defined by integration along a path in a two-dimensional plane:

$$
J_{k}=\int_{\Gamma}\left(W \delta_{j k}-\sigma_{i j} u_{i, k}\right) n_{j} d \Gamma
$$

where $i, j, k$ are the Einstein indices, $J_{k}$ is the component of the J-integral for the crack opening in the $x_{k}$ direction, $W$ is the elastic energy density, $\delta_{j k}$ is the Kronecker delta, 
$\sigma_{i j}$ is the stress component, $u_{i, k}$ is the derivative of the $i^{\text {th }}$ component of the displacement vector with respect to the $k$, and $n_{j}$ is $j$-component of outward normal. The importance of the J-integral is aligned with the fact that it is independent of the path around a crack and that it can be used as a fracture criterion and also can be related to the crack-tip opening displacement. Under opening-mode loading, the criterion for the crack initiation takes the form of $J=J_{c}$, where $J_{c}$ is a material property for a given thickness under the specified conditions [93].

The concept of stress intensity factor was introduced by Irwin in 1957 [94]. SIF is a measure of the magnitude of the stress field near the crack tip and depends on the geometrical configuration and the applied loading conditions of the body. Three types of SIFs are associated with each of the three crack opening modes. The first stress intensity factor is $K_{I}=\lim _{r \rightarrow \infty, \theta=0} \sigma_{y y} \sqrt{2 \pi r}$, which can be simplified to $K_{I}=\sigma_{\infty} \sqrt{\pi} a$ for the central crack of width $2 a$ in an infinite domain and $K_{I}=1.12 \sigma_{\infty} \sqrt{\pi} a$ for the edge crack of length $a$ in an infinite domain. The unstable fracture occurs when one of the stress intensity factors $K_{i}$ reaches its critical value $K_{i c}$. The critical value $K_{i c}$ is called fracture toughness and represents the potential ability of the material to withstand a given stress field at the tip of a crack [51]. The described SIF-based crack growth criterion is called Irwin's approach.

In linear elastic fracture mechanics, the stress and displacement components at the crack tip are characterized by the stress intensity factors $K_{I}, K_{I I}$, and $K_{I I I}$. Considering mixed-mode loading and the integration over the circle with the crack as its center, the J-integral is related to the stress intensity factors [93]:

$$
\begin{gathered}
J_{1}=\frac{(k+1)(1+\nu)}{4 E}\left(K_{I}^{2}+K_{I I}^{2}\right)+\frac{(1+\nu)}{E} K_{I I I}^{2}, \\
J_{2}=\frac{(k+1)(1+\nu)}{2 E} K_{I} K_{I I},
\end{gathered}
$$

where $k+1=4 /(1+\nu)$ for plane stress and $k+1=4-4 \nu$ for plane strain. For the 
Mode I crack loading, J-integral is equivalent to the energy release rate $G$ :

$$
J_{1}=J=\frac{(k+1)(1+\nu)}{4 E} K_{I}^{2}=G .
$$

In general, the J-integral is equal to the energy release rate $G$ if the non-elastic zone reduces to a point at the interior of the integral boundary, the crack faces are traction-free, and the crack is plane and extends in its own direction [95].

\subsubsection{Fracture mechanics in the Cosserat theory of elasticity}

In the area of linear elastic fracture mechanics for the Cosserat materials, several important works should be mentioned: analytical studies of the path-independent integrals and higher order crack modes for Cosserat continua [96, 97], experimental studies of short cracks and stress concentration factors in bones [35, 11], and analytical studies of the asymptotic fields solutions in the vicinity of a crack tip [98, 99, 100, 101]. Nevertheless, due to the specifics and complexity of the problem, the computations of the stress intensity factors and the relations between SIFs and the J-integral are not presented in full in these works. The absence of these relations causes difficulties for the numerical implementation of the fracture concepts in the Cosserat model. In the current work, the edge crack opening model in the Cosserat material is presented, the methods of implementation are discussed, and the dependence of the solution from the coupling level is introduced.

There are two approaches to consider cracks in the micropolar materials. The first approach, which is adopted in this study, is based on the classical linear elastic fracture mechanics and assumes conventional I-III crack opening modes [102]. According to the second approach, the presence of the additional degrees of freedom leads to the cracks of higher modes IV-VI, also called bending cracks [96, 97]. These crack modes describe the discontinuities in the corresponding components of the Cosserat rotations and produce the concentrations of the moment stresses at a crack tip. However, the latter theory does not have sufficient information about fracture parameters for the higher mode cracks, such as additional stress intensity factors. 
The asymptotic fields for the displacements near a crack tip for Mode I crack opening in a Cosserat medium are presented by Diegele et al. in [98]:

$$
\begin{aligned}
{\left[\begin{array}{c}
u_{r}^{t i p} \\
u_{\theta}^{t i p}
\end{array}\right] } & =\sqrt{\frac{r}{2 \pi}} \frac{\tilde{K}_{I}}{\mu[\mu+\alpha(3-2 \nu)]} \\
& {\left[\begin{array}{c}
\cos \frac{\theta}{2} \mu(1-2 \nu)-\alpha+[\mu+\alpha(7-6 \nu)] \sin ^{2} \frac{\theta}{2} \\
-\sin \frac{\theta}{2}\left[2(\mu+\alpha)(1-\nu)-[\mu+\alpha(7-6 \nu)] \cos ^{2} \frac{\theta}{2}\right.
\end{array}\right] } \\
& +r \frac{k_{I}}{2 \mu}\left[\begin{array}{c}
2\left(\cos ^{2} \theta-\nu\right) \\
-\sin (2 \theta)
\end{array}\right]+\left[\begin{array}{c}
O\left(r^{3 / 2}\right) \\
O\left(r^{3 / 2}\right)
\end{array}\right], \\
\phi^{t i p} & =\sqrt{\frac{2 r}{\pi}} \frac{L_{I}}{\gamma+\delta} \sin \frac{\theta}{2}+O\left(r^{3 / 2}\right),
\end{aligned}
$$

where $\tilde{K}_{I}$ and $L_{I}$ are the Mode I stress intensity factors for micropolar elasticity, and $k_{I}$ is a second order stress intensity factor. An interesting observation is that the couple stresses for the Mode I crack are singular, but the couple stresses for the Mode II are regular. SIFs are introduced in that work as

$$
\begin{aligned}
\tilde{K}_{I} & :=\lim _{r \rightarrow 0}\left\{\sqrt{2 \pi r} T_{\theta \theta}(r, \theta) \mid \theta=0\right\}, \\
L_{I} & :=\lim _{r \rightarrow 0}\left\{\sqrt{2 \pi r} M_{z \theta}(r, \theta) \mid \theta=0\right\} .
\end{aligned}
$$

The degree of coupling for the translations and rotations is governed by elastic constant $\alpha$. For $\alpha=0$ and $\alpha \rightarrow \infty$, the problem reduces to the classical linear elasticity and to the couple stress linear elasticity, respectively. Typical values for $\alpha$ for real materials vary from $\alpha=10^{-1}$ to $\alpha=10^{5}[98]$.

It is important to mention that $\tilde{K}_{I}$ and $L_{I}$ are not independent $[102,103]$. As well, it should be stressed that the special stress intensity factors for the micropolar theory of elasticity $\tilde{K}_{I}$ and $\tilde{K}_{I I}$ are distinct from the stress intensity factors of the classical theory 
of elasticity. The effect of material parameters on the $\frac{\tilde{K}_{I}}{K_{I}}$ and $L_{I}$ is presented in [98]. Depending on the $\alpha$ and $\gamma$ values, the deviation of $\tilde{K}_{I}$ from $K_{I}$ varies from $14 \%$ to $26 \%$. The distributions of $\frac{\tilde{K}_{I}}{K_{I}}$ and $L_{I}$ as a functions of $\frac{\alpha}{E}$, parametrized by $\gamma$, are presented in Figures 2.9-2.10. In addition, the studies of the Mode II stress intensity factors $\left(\frac{\tilde{K}_{I I}}{K_{I I}}\right)$ are presented in this paper.

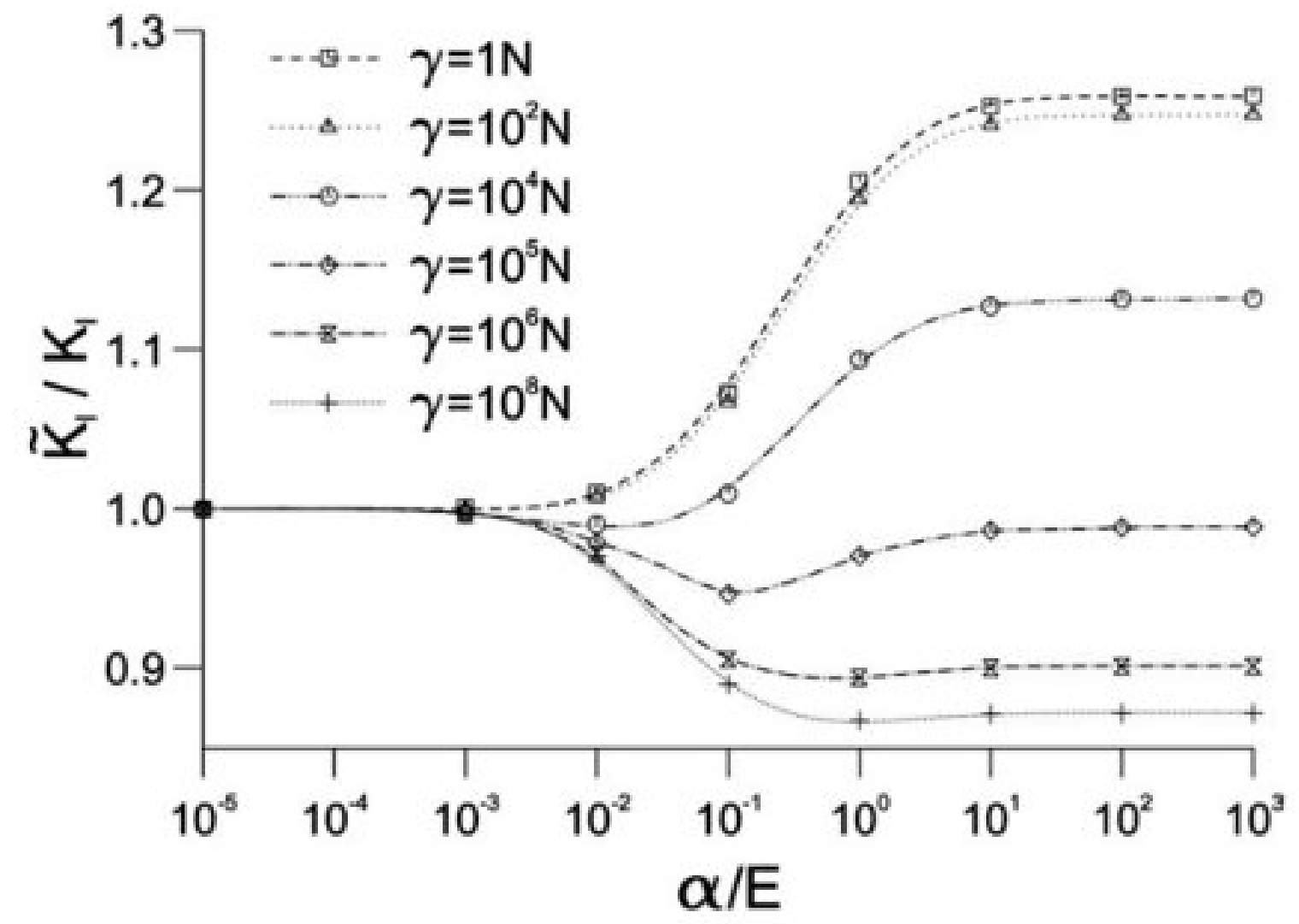

Figure 2.9: The representation of distribution of $\frac{\tilde{K}_{I}}{K_{I}}$ as a function of $\frac{\alpha}{E}$, parametrized by $\gamma$ (Diegele et al., 2004).

The first investigation in the area of fracture parameters in the Cosserat materials, such as stress concentration at the crack tip, was introduced in [103, 102]. In both papers, the authors discussed the effect of the couple stresses on the stress concentration at a crack tip and the mechanical behaviour in the vicinity of a crack tip. The main conclusion in [103] 


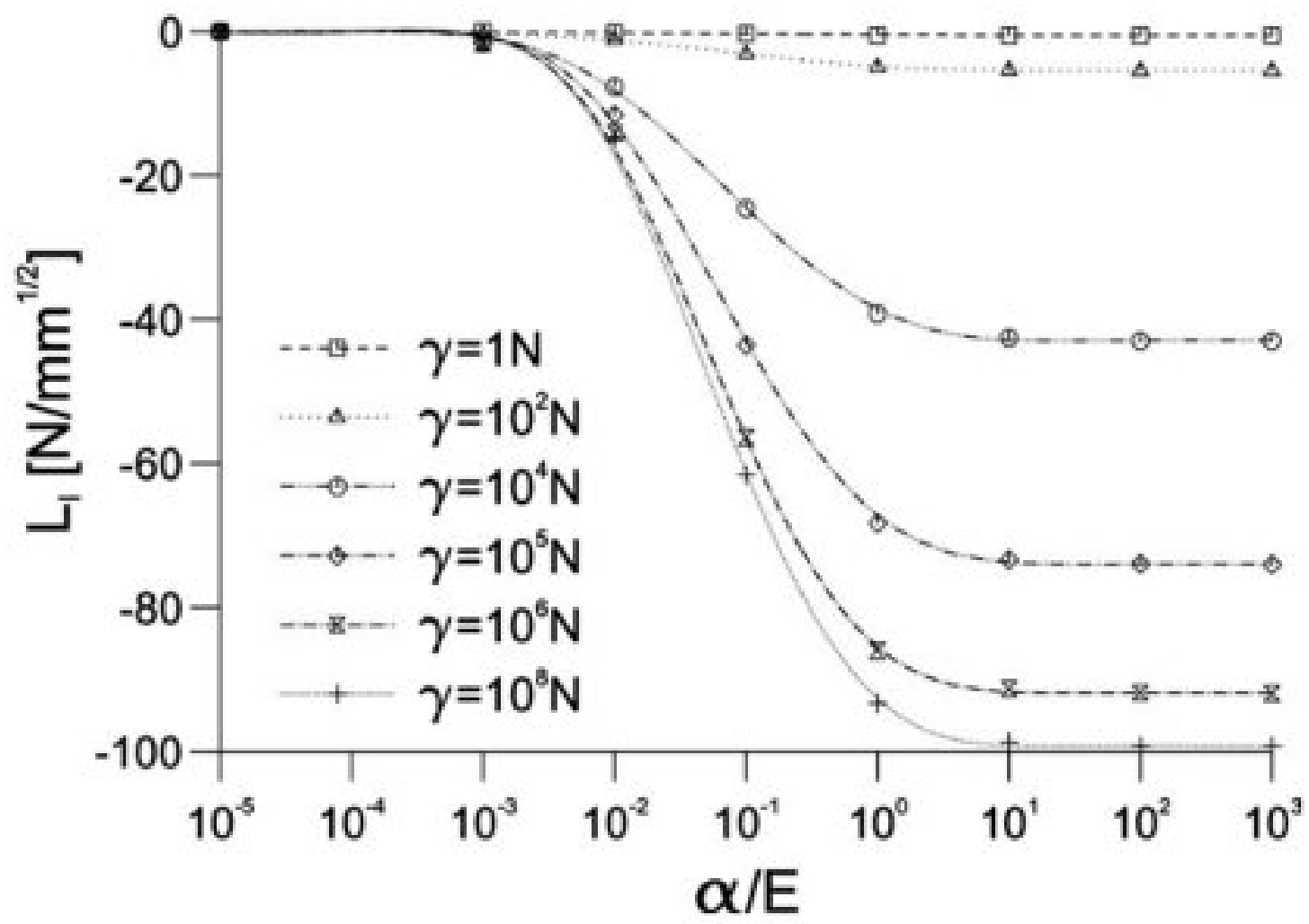

Figure 2.10: The representation of distribution of $L_{I}$ as a function of $\frac{\alpha}{E}$, parametrized by $\gamma$ (Diegele et al., 2004).

was that stress and couple stress fields had the same order of singularity as those in the problems of the classical theory of elasticity. In both papers, it was demonstrated that the energy release rate tended to the classical result when the micropolar parameter tended to zero and the stress intensity factor did not. In addition, the authors of [102] discussed the path-independence of the contour integral for the micropolar (the Cosserat model with free rotations) and couple stress (the Cosserat model with constrained rotations) cases. First, for the case of the Cosserat continuum with constant rotations, the energy release rate tended to the classical result as the characteristic length tended to zero, and the energy release rate decreased as the micropolar (or couple-stress) parameter increased. Second, they stated that the J-integral derived for the Cosserat problem was path-independent for 
both the micropolar and couple-stress problems. Another important observation was that the energy release rate tended to the classical elastic result as $\gamma$ parameter tended to zero.

The Cosserat equivalent to the J-integral has been derived in numerous ways [96, 97, 104, 105]. Following the derivation presented in [104], the energy release rate in the absence of the body forces and body moments for plane strain micropolar elasticity is

$$
J_{i}=\int_{\Gamma} P_{j i} n_{j} d \Gamma,
$$

where $i$ and $j$ are the Einstein indices, $\Gamma$ is the contour about the crack tip, and $\mathbf{n}$ is the normal to the contour $\Gamma$. $\mathbf{P}$ is the energy momentum tensor [106]:

$$
P_{i j}=W \delta_{u j}-\sigma_{i k} u_{k, j}-m_{i 3} \phi_{3, j}
$$

where $W$ is elastic energy per unit volume; $\sigma_{i k}$ and $m_{i 3}$ are the components of the stress and couple stress tensors, respectively; $u_{k, j}=\partial u_{k} / \partial x_{j}$ and $\phi_{3, j}=\partial \phi_{3} / \partial x_{j}$ are derivatives of the translations and rotations with respect to $x_{j}$, respectively. The elastic energy is defined as

$$
W=\frac{1}{2} \sigma_{i j} \varepsilon_{i j}+\frac{1}{2} m_{i j} \varkappa_{i j},
$$

where $\varepsilon_{i j}$ and $\varkappa_{i j}$ are the components of the strain and curvature tensors.

\subsection{Cosserat models coupled with the Finite Element Method}

In contrast to the coupled FEM/Cosserat models, the implementation of XFEM to the Cosserat elasticity models is not widely presented yet. Providas and Kattis in [107] introduced the solution of the boundary value problem in two dimensional linear isotropic Cosserat elasticity based on FEM. They reduced the three dimensional theory to the prob- 
lem of the plane strain expressing it in the rectilinear coordinates. After examining three types of elements, they showed that six-node quadratic/linear triangles produced a more accurate result than standard three-node linear triangles and the six-node quadratic triangles. Yang and Huang in [108] studied the effect of the technical constants of the micropolar medium (i.e., micropolar Young's modulus $E_{m}$, Poisson's ratio $\nu_{m}$, characteristic length $l$, coupling factor $N$, and micropolar elastic constants $\lambda, \mu, \alpha, \beta, \gamma, \kappa)$ on the Poisson's ratio $\nu$ for a rectangular plate using the finite element method. They identified few dependencies among the parameters: only the micropolar Poisson's ratio $\nu_{m}$ could alter the Poisson's ratio $\nu$ of the deformed plate, and changing of any of the other three micropolar elastic constants led to the change of the Poisson's ratio. Also, either a positive or negative Poisson's ratio $\nu$ of the deformed plate may be obtained by changing the micropolar Poisson's ratio $\nu_{m}$ due to the restrictions on the elastic constants. This result is useful for understanding the effect of the four Cosserat constants on the model.

Another important and wide implementation of the FEM/Cosserat models is found in the field of geomechanics. The FEM/Cosserat methods can effectively model the deformations in layered geomaterials, where the internal length scale is associated with the distance between the layers and their characteristics $[109,110,111]$. For these types of problems, it is necessary to use higher-order gradient theories, such as the Cosserat theory of elasticity, to incorporate a discontinuity in deformation at the interface of the layers and the consequent internal length scale into the governing equations. Riahi and Curran [6] introduced a successful implementation of the Cosserat continuum coupled with the FEM model to simulate layered materials. In that paper, the full three-dimensional elasto-plastic formulation of the Cosserat theory of elasticity was performed in the finite element code, and the dependence of accuracy of the result and model parameters (such as thickness, interaction of layers, number of layers, mesh size, boundary conditions) was studied. The main result showed good consistency with the analytical solution predicted by the plate theory and by the discrete element method technique. This method was capable of predicting the natural response of the layered geomaterials. The comparison of the Cosserat model and the explicit joint finite element model was presented in [111], and the extension of the Cosserat model to the elasto-plastic response of layered rocks was described in [112].

Another important benchmark in this field is three dimensional finite element modelling 
of shear band localizations using the Cosserat continuum developed by Khoei et al. [5]. The history-dependent micropolar material of elasto-plasticity was examined in this paper. The authors showed the capability of the FEM/Cosserat models to solve strain-softening problems and compared of the classical and the Cosserat models. With a decrease of the internal length parameter to zero, the solution converged to the classical elasticity result. However, with the non-zero internal length parameter, the Cosserat results were more accurate than those of the classical model.

\subsection{Cosserat models coupled with the eXtended Fi- nite Element Method}

Further implementation of the Cosserat models was done by coupling them with the extended finite element method.

Khoei and Karimi [4] proposed the combined Cosserat/XFEM model to simulate the strain localization in elasto-plastic solids. They enriched normal to the discontinuity displacement and assumed the tangential displacement and micro-rotations to be continuous. The proposed computational method was able to capture the strain localization even on the regular basic grid. In addition, Khoei et al. [113] simulated the non-linear behaviour of the materials (2D/3D large plasticity deformations) by representing independent of the finite element mesh material interfaces and accomplishing the process by integrating enriched elements with a larger number of Gauss points whose positions were fixed in the element.

Modelling of fracture processes in quasi-brittle materials, such as pervious concrete and rock masses, is an important area of the research. Simulation of cracks in a solid body is based on the continuum description. The material can be described using elastoplastic, damage mechanics, or coupled constitutive laws. The above mentioned materials have discontinuous structure, which can be described as a homogenized medium using the Cosserat elasticity theory. These models account for the elementary inter-block bending and provide a large-scale response of a medium. This fact is important for geomechanics problems. The characteristic length of the microstructure should be incorporated into the 
constitutive model and leads to the necessity to use the higher-order continuum models such as the Cosserat model. No coupled Cosserat/XFEM simulations for the cracks in the elastic body has been done to date.

In the current research, the combined Cosserat/XFEM model is presented to perform the fracture analysis in the Cosserat elastic solids. 


\section{Chapter 3}

\section{Overview of the FEM}

This section describes the construction of the approximations (the weight functions and trial solutions) for two dimensional elasticity problems.

\subsection{Overview}

The partial differential equations for the multi-dimensional practical problems in engineering generally have complex boundary conditions. Therefore, numerical approximation is often the only possible way to solve these equations. The finite element method is proven to be an effective modelling technique for a number of engineering problems.

The main approach of the finite element method is to approximate the weight functions and trial solutions by the finite element shape functions. The important characteristic of the FEM solution is that the quality of the solution improves with mesh refinement, or as polynomial order of the shape functions is increased. The FEM solution converges to the exact solution when the conditions of continuity and completeness are fulfilled. Continuity means that the trial solutions and weight functions are sufficiently smooth. For second order differential equations the weight functions and the trial solutions should be $C^{0}$ continuous at every point in the domain, including the interfaces of the elements. Completeness means that the trial and weight functions and their derivatives approximate 
the given smooth function with arbitrary accuracy, i.e are capable of assuming constant values. For example, in the context of elasticity, the finite elements should be able to represent rigid body motion and constant strain states exactly.

\subsection{Shape functions construction}

Consider an one dimensional element having two nodes, one at each end. The shape functions for the two-node element are shown in Figure 3.1. The linear polynomial approximation has the following form [114]:

$$
\theta^{e}(x)=a_{0}^{e}+a_{1}^{e} x .
$$

The approximation can be rewritten as

$$
\theta^{e}(x)=\boldsymbol{N}^{e}(x) \boldsymbol{d}^{e},
$$

where $\boldsymbol{N}^{e}(x)=\left[N_{1}^{e}(x) N_{2}^{e}(x)\right]$ is the element shape function matrix, and $\boldsymbol{d}^{e}=\left[\theta_{1}^{e} \theta_{2}^{e}\right]$ is the element nodal displacement matrix.

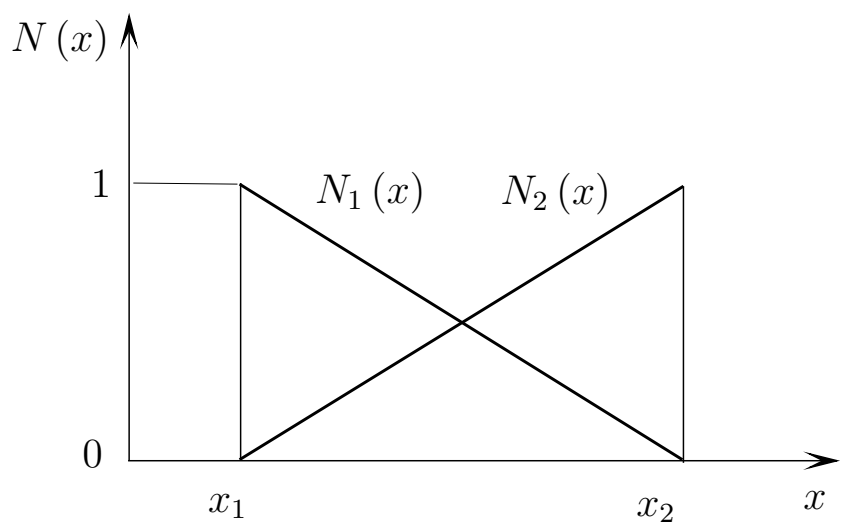

Figure 3.1: The representation of the shape functions for the two-node element.

The fundamental property of the shape functions is the interpolation property. This 
property means that the shape functions could be used as the interpolants to fit any data. The shape functions also obey Kronecker delta property:

$$
N_{I}^{e}\left(x_{J}^{e}\right)=\delta_{I J}
$$

where $x_{J}^{e}$ is the coordinate of the $J$-node of the element and $\delta_{I J}$ is the Kronecker delta defined as

$$
\delta_{I J}=\left\{\begin{array}{l}
1 \text { if } I=J \\
0 \text { if } I \neq J
\end{array} .\right.
$$

In two dimensional problems, the two types of the finite elements often used are triangular elements and quadrilateral elements. The three-node triangular elements or four-node quadrilateral elements have the nodes only at their vertices. The higher order elements have additional nodes on the edges.

The quadrilateral element is used to discretize the problem domain in the current research. The nodes are usually numbered counterclockwise in the quadrilateral element, starting with the left bottom node. Considering the rectangular element, the element approximation function of the spacial coordinates $(x, y)$ is

$$
\theta^{e}(x, y)=a_{0}^{e}+a_{1}^{e} x+a_{2}^{e} y+a_{3}^{e} x y
$$

where the $a_{0}^{e}, a_{1}^{e}, a_{2}^{e}, a_{3}^{e}$ are the coefficient of the polynomial, chosen so that the $C^{0}$ continuity between the two elements is satisfied.

The element shape functions $\boldsymbol{N}^{e}$ for the rectangular element are constructed by the tensor product method. They are obtained by the product of the one dimensional shape functions obeying the Kronecker delta property:

$$
N_{[I, J]}^{e}=N_{I}^{e}(x) N_{J}^{e}(y)
$$

where $I=1,2$ and $J=1,2$. 
Two dimensional shape functions for the rectangular element are

$$
\begin{gathered}
N_{1}^{e}(x, y)=\frac{1}{A^{e}}\left(x-x_{2}^{e}\right)\left(y-y_{4}^{e}\right), \\
N_{2}^{e}(x, y)=-\frac{1}{A^{e}}\left(x-x_{1}^{e}\right)\left(y-y_{4}^{e}\right), \\
N_{3}^{e}(x, y)=\frac{1}{A^{e}}\left(x-x_{1}^{e}\right)\left(y-y_{1}^{e}\right), \\
N_{4}^{e}(x, y)=-\frac{1}{A^{e}}\left(x-x_{2}^{e}\right)\left(y-y_{1}^{e}\right),
\end{gathered}
$$

where $A^{e}$ is the area of the element and $x^{e}$ and $y^{e}$ are the coordinates of the element nodes. The representation of the rectangular element shape functions is shown in Figure 3.2.
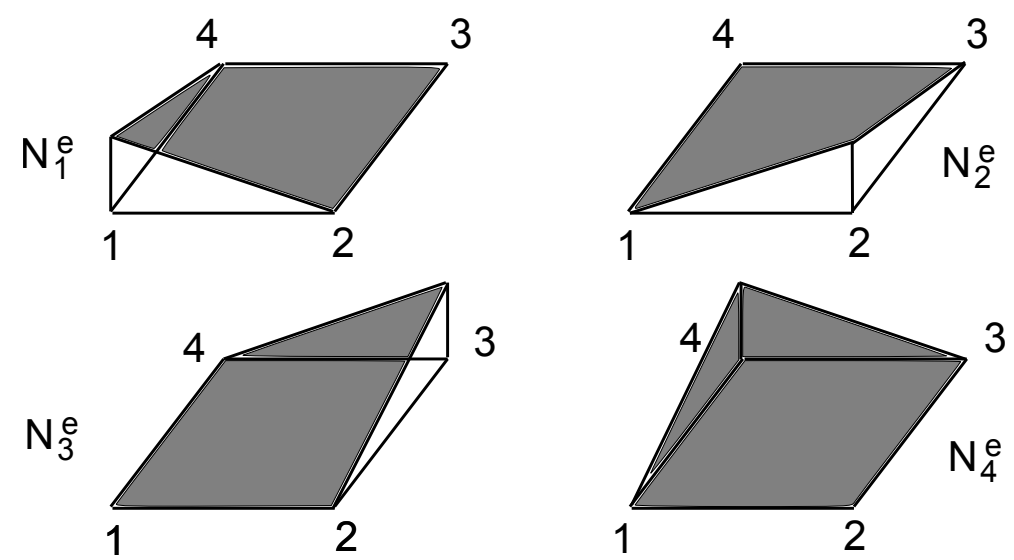

Figure 3.2: The representation of the shape functions for the rectangular element in 2D.

FEM may employ parametric or isoparametric representation of the functions [114]. If the functions are linear along the edges of an arbitrary element and the $C^{0}$ continuity between the element is held between the two common nodes, the parametric (or direct) construction of the shape functions may be employed, as discusses above. To construct quadrilateral elements or the elements with the curved sides, the isoparametric shape functions should be used. This method allows to model the structures with the complex geometries, which is especially important for the practical engineering.

The isoparametric concept is described as the method of mapping from the parent $(\eta, \xi)$ to the physical Cartesian coordinates $(x, y)$ (Figure 3.3). The approximation function $\theta$ 


\begin{tabular}{|c|c|c|}
\hline Node $I$ & $\xi_{I}$ & $\eta_{I}$ \\
\hline 1 & -1 & -1 \\
\hline 2 & 1 & -1 \\
\hline 3 & 1 & 1 \\
\hline 4 & -1 & 1 \\
\hline
\end{tabular}

Table 3.1: Nodal coordinates in the parametric element domain.

is presented in terms of the parent coordinates first, rather than in terms of the global coordinates $(x, y)$ :

$$
\theta^{e}(\eta, \xi)=a_{0}^{e}+a_{1}^{e} \xi+a_{2}^{e} \eta+a_{3}^{e} \xi \eta
$$

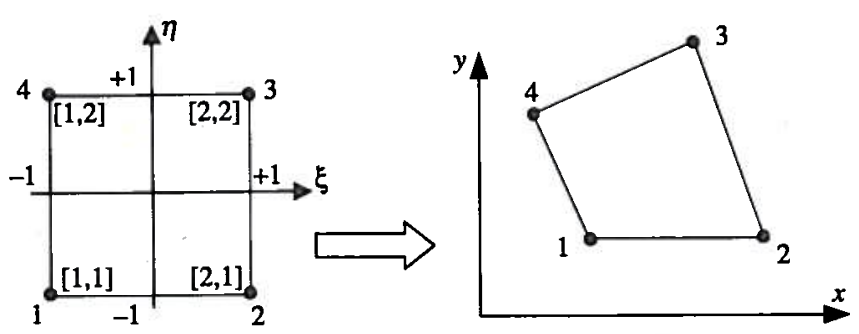

Figure 3.3: Mapping from the parent coordinates to the physical coordinates (Fish, Belytschko. A First Course in Finite Elements).

The shape functions have form of

$$
N_{I}^{4 Q}(\eta, \xi)=\frac{1}{4}\left(1+\xi_{I} \xi\right)\left(1+\eta_{I} \eta\right)
$$

where $\left(\eta_{I}, \xi_{I}\right)$ are the nodal coordinates in the parent element, presented in Table 3.2.

The trial solution is

$$
\theta^{e}(\eta, \xi)=\boldsymbol{N}^{4 Q}(\eta, \xi) \boldsymbol{d}^{e}
$$

where $\boldsymbol{d}^{e}$ is the nodal displacement vector. 
The physical coordinates are mapped by the same functions as those for the approximation. This property is an essential feature of the isoparametric element:

$$
\begin{aligned}
& x(\eta, \xi)=\boldsymbol{N}^{4 Q}(\eta, \xi) \boldsymbol{x}^{e}, \\
& y(\eta, \xi)=\boldsymbol{N}^{4 Q}(\eta, \xi) \boldsymbol{y}^{e},
\end{aligned}
$$

where $\boldsymbol{x}^{e}$ and $\boldsymbol{y}^{e}$ denotes the $x$ and $y$ coordinates of the element nodes.

The approximation constructed by quadrilateral elements is uniquely determined along the edge of the element and is $C^{0}$ continuous $[114,115]$.

\subsection{Gauss quadrature}

Gauss quadrature is the numerical integration technique for the polynomial or nearly polynomial functions. The Gauss integration technique and formulas for the parent domain $(\eta, \xi)$ are presented in [114]. Following this technique, first, the physical domain $(x, y)$ should be mapped to the parent domain. Consider the integral over the domain $\Omega$ :

$$
I=\int_{\Omega^{e}} f(\eta, \xi) d \Omega .
$$

After the integration over the $\xi$ and $\eta$ and summation over the domain $\Omega$, the equation (3.16) is evaluated as

$$
I=\sum_{i=1}^{n_{g p}} \sum_{j=1}^{n_{g p}} W_{i} W_{j}\left|\boldsymbol{J}^{e}\left(\xi_{i}, \eta_{j}\right)\right| f\left(\xi_{i}, \eta_{j}\right),
$$

where $n_{g p}$ is number of Gauss quadrature points, $W_{i}$ and $W_{j}$ are the weights of the corresponding quadrature points, and $\boldsymbol{J}^{e}$ is Jacobian matrix defined as

$$
\boldsymbol{J}^{e}=\operatorname{det}\left[\begin{array}{ll}
\frac{\partial x}{\partial \xi} & \frac{\partial y}{\partial \xi} \\
\frac{\partial x}{\partial \eta} & \frac{\partial y}{\partial \eta}
\end{array}\right] .
$$


Gauss quadrature weights and points are defined for all of the major types of the elements and could be found in [114].

\subsection{Convergence of FEM}

\subsubsection{Errors in the approximation}

The errors of the finite element solution of a differential equation can be grouped into three main categories [115]:

- Boundary errors due to the approximation of the domain. They occur in the two dimensional problems on the non rectangular domain, for which the solution is obtained on the modified domain.

- Errors due to the numerical evaluation of the integrals and numerical computation or round-off errors, which occur in the computation of the stiffness matrix, force vectors and in the post-processing.

- Errors due to the approximation of the solution.

First type of errors approaches zero with the mesh refinement, because the domain is being more accurately presented. Errors of the second type are small comparing to the approximation errors in the most linear problems. Therefore, the approximation error is an important quantity, which mainly defines the accuracy and the convergence properties of the FEM/XFEM solution.

\subsubsection{Measures of the errors}

The evaluation of the error in the solution is generally performed with the help of norms. The norm is the distance between two functions - the finite element approximated solution and the exact solution. In order to calculate the difference between these two functions the L2 and energy norms are employed. 
The L2 norm is defined separately for translations and rotations as

$$
\begin{aligned}
& \left\|\boldsymbol{u}_{e x}-\boldsymbol{u}_{h}\right\|_{L 2}=\left(\int_{\Omega}\left(\boldsymbol{u}_{e x}-\boldsymbol{u}_{h}\right)^{2}\right)^{1 / 2}=\left(\int_{\Omega}\left(\left(u_{1 e x}-u_{1 h}\right)^{2}+\left(u_{2 e x}-u_{2 h}\right)^{2}\right) d \Omega\right)^{1 / 2} \\
& \left\|\phi_{e x}-\phi_{h}\right\|_{L 2}=\left(\int_{\Omega}\left(\phi_{e x}-\phi_{h}\right)^{2} d \Omega\right)^{1 / 2}
\end{aligned}
$$

where $\boldsymbol{u}_{e x}$ and $\phi_{e x}$ are the analytical solutions, and $\boldsymbol{u}_{h}$ and $\phi_{h}$ are the corresponding numerical solutions.

The energy norm is defined as

$$
\left\|\boldsymbol{u}_{e x}-\boldsymbol{u}_{h}\right\|_{E}=\left(0.5 \int_{\Omega}\left(\varepsilon_{e x}-\varepsilon_{h}\right)^{\top} \boldsymbol{D}^{u}\left(\varepsilon_{e x}-\varepsilon_{h}\right) d \Omega+0.5 \int_{\Omega}\left(\varkappa_{e x}-\varkappa_{h}\right)^{\top} \boldsymbol{D}^{\phi}\left(\varkappa_{e x}-\varkappa_{h}\right) d \Omega\right)^{1 / 2}
$$

where $\boldsymbol{u}, \boldsymbol{\varepsilon}$ and $\varkappa$ with the superscript "ex" are the exact solutions of displacement, strain and curvature, and with the superscript " $h$ " are the corresponding numerical solutions.

The L2 norm and the energy norm are normalized, respectively, as

$$
\begin{gathered}
\frac{\left\|\boldsymbol{u}_{e x}-\boldsymbol{u}_{h}\right\|_{L 2}}{\left\|\boldsymbol{u}_{e x}\right\|_{L 2}} \\
\frac{\left\|\boldsymbol{u}_{e x}-\boldsymbol{u}_{h}\right\|_{E}}{\left\|\boldsymbol{u}_{e x}\right\|_{E}}
\end{gathered}
$$

\subsubsection{Accuracy of the solution}

Error estimation shows how rapidly the approximated solution is converging to the exact solution [114]. Consider the function to be approximated as a complete polynomial of order $p$. Using the L2-norm, the error in the function could be presented as:

$$
\|e\|_{L_{2}}=C h^{p+1}
$$


Using the energy norm, the error in the function could be presented as:

$$
\|e\|_{E}=C h^{p} .
$$

Considering the domain without the discontinuities, for the finite element approximation with the linear element (the order of polynomial $p=1$ ), the optimal convergence rate is 2 in L2-norm and 1 in energy norm.

The crack in domain introduces the singularities in the stress and strain fields. This fact leads to the reduced convergence rates of 1 in the L2-norm and 0.5 in the energy norm in the FEM solution. On the other hand, the XFEM solution achieves the optimal convergence rate of 2 in the L2-norm and 1 in the energy norm [116].

\subsection{Verification of FEM}

In order to verify the finite element code the following test may be conducted:

- Multielement displacement patch test

- Multielement force patch test

- Patch test with the known exact solution or the manufactured solution

- Convergence study

In order to fulfil the requirements of the compatibility, the multielement patch test is introduced. The patch is a set of elements attached to a given node, which is called a patch node. The main idea of the patch test is that the simple problem must be solved exactly by the proper elements. The simple problem could be introduced as a displacement or force patch test. The displacement patch test applies the boundary displacement to the patch and verifies that the patch reproduces exactly the rigid body motion and constant strain state. The force patch test applies the boundary forces and verifies that the patch reproduces the exact constant stress state. 
For more complicated problems, the patch test with the known analytical solution or manufactured solution should be employed. In this case, the exact displacements are applied on the boundaries of the two dimensional domain, and the finite element approximation should reproduce the exact solution inside the domain. The convergence study then shows how close the approximation is to the exact solution with the help of the error measure, introduced above. With the mesh refinement the approximated solution must tend to the exact solution with the optimal convergence rate for a given problem.

\subsubsection{Computation scheme for the error evaluation of the XFEM solution with the FEM exact solution}

In the present research, the error evaluation of the XFEM solution was performed according to the following steps:

1. Defining the model Define the boundary of the physical model, the boundary and loading condition, the material model.

2. Front tracking Define the crack geometry (front) using level set method.

3. FEM solver Obtain the FEM solution of the problem for a very fine mesh.

4. Saving FEM results Save the reference results of the FEM computation: nodes information, connectivity matrix, assigned degrees of freedom, displacement vector.

5. XFEM solver Define mesh sizes sequence.

6. Obtain the XFEM solution of the problem for a given mesh.

7. Post-processing Load the FEM solution results. Load the XFEM solution results.

8. For $\mathrm{n}=1$ to number of elements, define the element quadrature order depending on the element enrichment type (Heaviside enrichment, branch functions enrichment, no enrichment). 
9. For $\mathrm{q}=1$ to number of quadrature points in the element, obtain the global coordinates of the quadrature point.

XFEM

Obtain the vector of element nodal displacements from the XFEM solution (step 6).

Calculate the strain and the curvature at the point - approximated values.

\section{FEM}

Define the element in the FEM mesh containing the point, using the global coordinates of the quadrature point.

Obtain the vector of element nodal displacements from the FEM solution (step 3).

Calculate the strain and the curvature - exact values.

Calculate the relative error in the energy using the equation (3.21).

10. Got to the step 9 .

11. Go to the step 8 .

12. Go to the next mesh for the XFEM solver (step 6). 


\section{Chapter 4}

\section{XFEM Model of the Crack in the Cosserat Elastic Material}

In this section, the governing equations are presented, the strong and the weak forms for a crack in a Cosserat medium are developed, the discrete equations are derived, the crack opening model in a Cosserat medium is described, and the J-integral computational scheme is presented.

\subsection{Governing equations}

Consider the two dimensional domain $\Omega$ bounded by $\Gamma$, as shown in Figure 4.1. Boundary $\Gamma$ consists of the sets $\Gamma_{u}, \Gamma_{\phi}, \Gamma_{t}$, and $\Gamma_{m}$, where displacements $\overline{\mathbf{u}}$, microrotations $\bar{\phi}$, surface forces (traction) $\overline{\mathbf{t}}$, and surface couple forces (moment) $\overline{\mathbf{M}}$ are prescribed, respectively. The internal surface of crack is denoted by $\Gamma_{c r}$. Boundary properties are as follows:

$$
\begin{aligned}
& \Gamma_{u} \cap \Gamma_{t}=\emptyset \text { and } \Gamma_{u} \cup \Gamma_{t}=\Gamma, \\
& \Gamma_{\phi} \cap \Gamma_{M}=\emptyset \text { and } \Gamma_{\phi} \cup \Gamma_{M}=\Gamma .
\end{aligned}
$$

The case of plane strain is considered. The components of displacement and rotations 


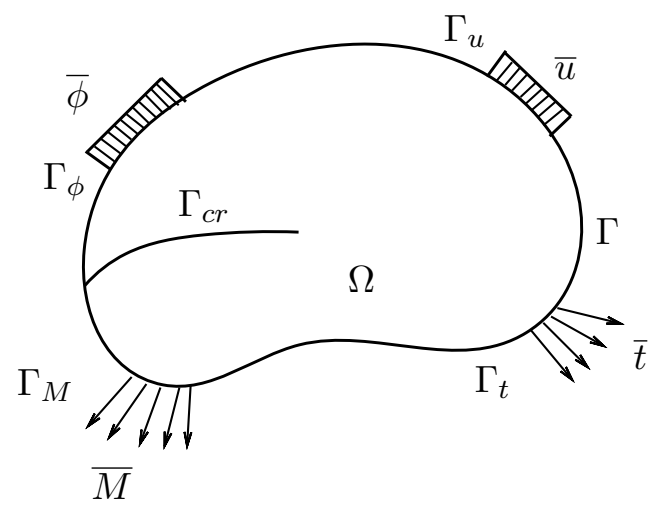

Figure 4.1: Body with external and internal boundaries subjected to loads.

are $\boldsymbol{u} \equiv\left(u_{1}, u_{2}, 0\right)$ and $\boldsymbol{\phi} \equiv\left(0,0, \phi_{3}\right)$, respectively.

In two dimensions, governing equilibrium equations are

$$
\begin{aligned}
& \nabla \cdot \boldsymbol{\sigma}+\mathbf{b}^{\sigma}=0, \\
& \nabla \cdot \mathbf{m}+b^{m}+\hat{\sigma}=0
\end{aligned}
$$

where $\boldsymbol{\sigma}$ is the Cauchy stress, $\mathbf{b}^{\sigma}$ is the external forces, $\mathbf{m}$ is the couple force stress, $b^{m}$ is the external moment, and $\hat{\sigma}=\sigma_{21}-\sigma_{12}$.

The Neumann boundary conditions are

$$
\begin{aligned}
\boldsymbol{\sigma} \cdot \mathbf{n} & =\overline{\mathbf{t}} \text { on } \Gamma_{t}, \\
\mathbf{m} \cdot \mathbf{n} & =\overline{\mathbf{M}} \text { on } \Gamma_{M},
\end{aligned}
$$

where $\mathbf{n}$ is the unit outward normal to $\Gamma$.

The Dirichlet boundary conditions are

$$
\begin{aligned}
\mathbf{u} & =\overline{\mathbf{u}} \text { on } \Gamma_{u}, \\
\phi & =\bar{\phi} \text { on } \Gamma_{\varphi} .
\end{aligned}
$$


The boundary conditions on the crack surface are

$$
\boldsymbol{\sigma} \cdot \boldsymbol{n}_{c r}=0 \text { and } \boldsymbol{m} \cdot \boldsymbol{n}_{c r}=0 \text { on } \Gamma_{c r},
$$

where $\boldsymbol{n}_{c r}$ is the normal to the crack surface $\Gamma_{c r}$.

The translations, $\boldsymbol{u}$, and the microrotations, $\phi$, are taken to be the independent variables. The strain, $\boldsymbol{\varepsilon}$, and the curvature, $\varkappa$, vectors have the following relationships with these variables:

$$
\begin{aligned}
\varepsilon & =\nabla \boldsymbol{u}-\hat{\boldsymbol{\phi}}, \\
\varkappa & =\boldsymbol{\nabla} \phi,
\end{aligned}
$$

where $\hat{\boldsymbol{\phi}}=\left[\begin{array}{cc}0 & -\phi_{3} \\ \phi_{3} & 0\end{array}\right]$.

The linear constitutive equations for the coupled model are

$$
\begin{aligned}
\boldsymbol{\sigma} & =\boldsymbol{D}^{u}: \boldsymbol{\varepsilon}, \\
\boldsymbol{m} & =\boldsymbol{D}^{\phi}: \varkappa,
\end{aligned}
$$

where $\boldsymbol{D}^{u}$ and $\boldsymbol{D}^{\phi}$ are the elastic moduli tensors which for an isotropic material are defined as

$$
\boldsymbol{D}^{u}=\left[\begin{array}{cccc}
2 \mu+\lambda & \lambda & 0 & 0 \\
\lambda & 2 \mu+\lambda & 0 & 0 \\
0 & 0 & \mu-\alpha & \mu+\alpha \\
0 & 0 & \mu+\alpha & \mu-\alpha
\end{array}\right]
$$

and

$$
\boldsymbol{D}^{\phi}=\left[\begin{array}{cc}
\gamma+\kappa & 0 \\
0 & \gamma+\kappa
\end{array}\right]
$$

where $\mu, \lambda, \alpha, \gamma$ and $\kappa$ are material constants. 


\subsection{Weak form}

The spaces of admissible displacement fields are

$$
\begin{aligned}
& U=\left\{\mathbf{u} \in H^{1}, \mathbf{u}=\overline{\mathbf{u}} \text { on } \Gamma_{u}, \mathbf{u} \text { is discontinuous on } \Gamma_{c r}\right\} \\
& \Phi=\left\{\phi \in H^{1}, \phi=\bar{\phi} \text { on } \Gamma_{\phi}, \phi \text { is discontinuous on } \Gamma_{c r}\right\}
\end{aligned}
$$

The test functions spaces are

$$
\begin{aligned}
& U_{0}=\left\{\mathbf{w} \in H^{1}, \mathbf{w}=0 \text { on } \Gamma_{u}\right\}, \\
& \Phi_{0}=\left\{v \in H^{1}, v=0 \text { on } \Gamma_{\phi}\right\}
\end{aligned}
$$

The weak forms of the equilibrium equations are constructed by multiplying the governing equations (4.3) and (4.4) by an arbitrary functions $\mathbf{w} \in \mathbf{U}_{\mathbf{0}}$ and $v \in \boldsymbol{\Phi}_{\mathbf{0}}$, respectively, and integrating over the domain $\Omega$. After integrating by parts and substituting the constitutive model for the stresses (4.12) and (4.13), we have the weak form of coupled boundary value problem of Cosserat elasticity: Find $\mathbf{u} \in U$ and $\phi \in \Phi$ such that

$$
\begin{aligned}
\int_{\Omega} \boldsymbol{\nabla} \boldsymbol{w}: \boldsymbol{\sigma} d \Omega & =\int_{\Omega} \boldsymbol{w} \cdot \boldsymbol{b}^{\sigma} d \Omega+\int_{\Gamma_{t}} \boldsymbol{w} \cdot \overline{\boldsymbol{t}} d \Gamma, \quad \forall \boldsymbol{w} \in U_{0} \\
\int_{\Omega} \boldsymbol{\nabla} v \cdot \boldsymbol{m} d \Omega & =\int_{\Omega} v b^{m} d \Omega+\int_{\Gamma_{M}} v \bar{M} d \Gamma, \quad \forall v \in \Phi_{0} .
\end{aligned}
$$

It was shown in [57] that the weak form is equivalent to the strong form.

\subsection{Discrete equations}

In the following subsection we will develop the discrete equations for a domain with a single crack. The extension to the case of multiple cracks follows in a straightforward manner.

Consider a crack $\Gamma_{c r}$ passing through a discretized domain, as shown in Figure 4.2. 


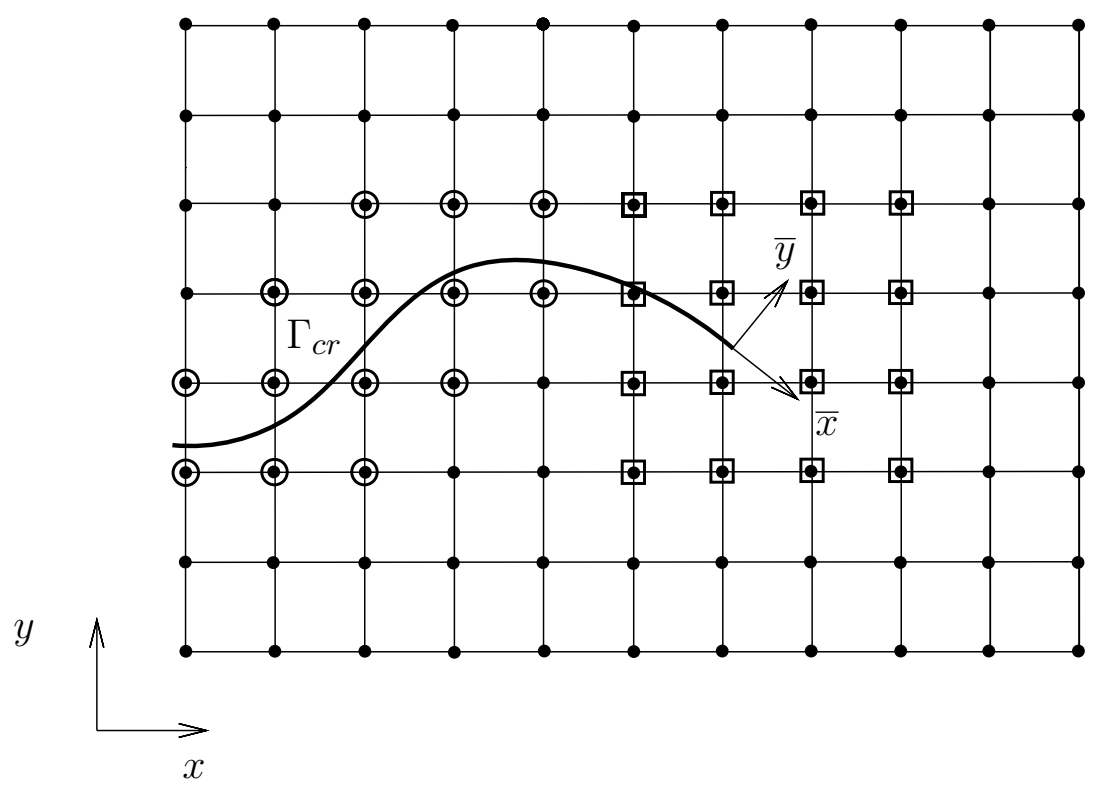

Figure 4.2: Illustration of a two dimensional domain containing a crack $\Gamma_{c r}$. The circled nodes are enriched by the Heaviside function, and the squared nodes are enriched by the crack tip enrichment function.

The level set method is employed to represent the crack position in the domain and track the propagation of the crack. The level set allows to describe the complicated crack geometry by a functional representation. According to the [75], the crack is considered as a one-dimensional curve in the two-dimensional space. The signed distance function is defined as

$$
f(\boldsymbol{x})= \pm \min \left\|\boldsymbol{x}-\boldsymbol{x}_{\Gamma_{c r}}\right\|
$$

where $\boldsymbol{x}$ is a point in the domain, $\boldsymbol{x}_{\Gamma_{c r}}$ is the point on the crack surface, and the sign is different on the two sides of the crack surface.

The crack surface $\Gamma_{c r}$ is represented as the zero level set of a function $f(\boldsymbol{x})$. The crack tip position is represented by the zero level set of a function $g(\boldsymbol{x})$, which intersects the crack tip and is orthogonal to the zero level set of a function $f(\boldsymbol{x})$. The signs of the distance for the $f(\boldsymbol{x})$ and $g(\boldsymbol{x})$ functions are shown in Figure 4.3. The crack is defined at 


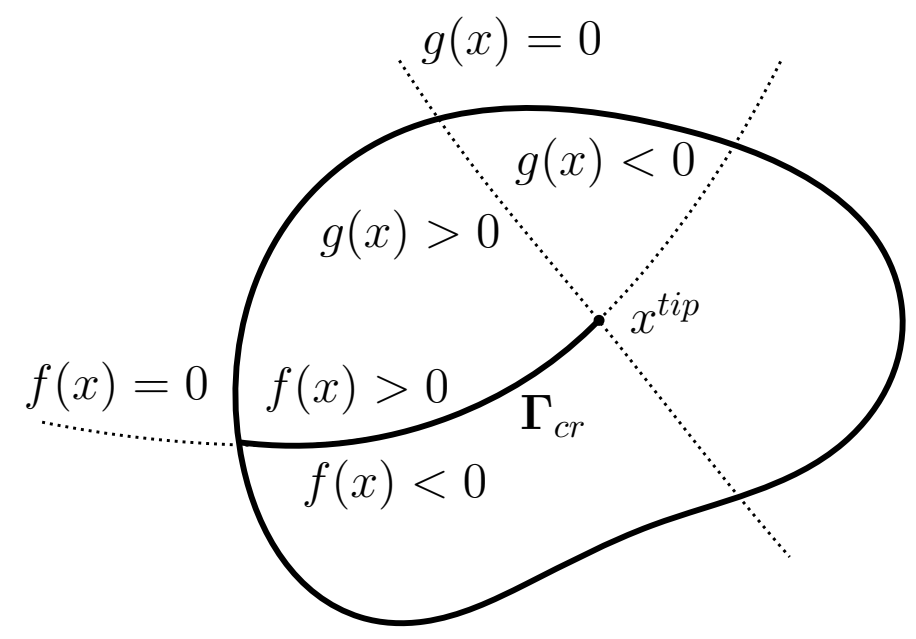

Figure 4.3: Level set method for the crack growth modelling.

the locations where the following conditions are true:

$$
\begin{aligned}
& f(\boldsymbol{x})=0, \\
& g(\boldsymbol{x}) \leq 0
\end{aligned}
$$

This method allows to assign discrete values of level set functions at each node of the elements. Thus, the level set functions $f^{h}(\boldsymbol{x})$ and $g^{h}(\boldsymbol{x})$ are the approximations of the signed distance functions $f(\boldsymbol{x})$ and $g(\boldsymbol{x})$ :

$$
\begin{aligned}
f^{h}(\boldsymbol{x}) & =\sum_{I} N_{I}(\boldsymbol{x}) \boldsymbol{f}_{I}, \\
g^{h}(\boldsymbol{x}) & =\sum_{I} N_{I}(\boldsymbol{x}) \boldsymbol{g}_{I},
\end{aligned}
$$

where $\boldsymbol{N}(\boldsymbol{x})$ are the standard bilinear finite element shape function.

The level set function representing the initial crack is constructed by computing the signed-distance function for the crack. The initial crack is then extended tangentially from its tip, and the signed-distance function is constructed from this extended crack. Crack growth is modelled by appropriately updating the $f(\boldsymbol{x})$ and $g(\boldsymbol{x})$ functions. 
Following a procedure similar to the original XFEM [69]:

$$
\begin{aligned}
\boldsymbol{u}^{h}(\boldsymbol{x}) & =\sum_{I \in S} N_{I}(\boldsymbol{x}) \boldsymbol{d}_{I}^{u}+\sum_{J \in S_{c r}} N_{J}(\boldsymbol{x})\left[H(f(\boldsymbol{x}))-H\left(f\left(\boldsymbol{x}_{J}\right)\right)\right] \mathbf{a}_{J}^{u}+ \\
& +\sum_{K \in S_{t i p}} N_{K}(\boldsymbol{x}) \sum_{m=1}^{M}\left[F_{m}(r, \theta)-F_{m}\left(r_{n}, \theta_{n}\right)\right] \boldsymbol{b}_{m K}^{u}, \quad \forall \boldsymbol{x} \in \Omega \\
\phi^{h}(\boldsymbol{x}) & =\sum_{I \in S} N_{I}^{\phi}(\boldsymbol{x}) d_{I}^{\phi}+\sum_{J \in S_{c r}} N_{J}^{\phi}(\boldsymbol{x})\left[H(f(\boldsymbol{x}))-H\left(f\left(\boldsymbol{x}_{J}\right)\right)\right] a_{J}^{\phi}+ \\
& +\sum_{K \in S_{t i p}} N_{K}^{\phi}(\boldsymbol{x})\left[F^{\phi}(r, \theta)-F^{\phi}\left(r_{n}, \theta_{n}\right)\right] b_{K}^{\phi}, \quad \forall \boldsymbol{x} \in \Omega
\end{aligned}
$$

where $S$ is the set of all nodes, $S_{c r}$ is the set of nodes with support crossed by the crack faces, $S_{t i p}$ is the set of nodes inside the predefined area around the crack tip, $N_{I}$ are the standard finite element shape functions for the vector field (translations), $N_{I}^{\phi}$ are the standard finite element shape functions for the scalar field (microrotations), $f(\mathbf{x})$ is signed distance to $\Gamma_{c r}, \mathbf{d}_{I}^{u}$ and $d_{I}^{\phi}$ are the standard FEM nodal translational and rotational degrees of freedom, $\mathbf{a}_{J}^{u}$ and $a_{J}^{\phi}$ are the Heaviside function enriched degrees of freedom, and $\mathbf{b}_{K}^{u}$ and $b_{K}^{\phi}$ are the branch function enriched degrees of freedom. $S_{c r}$ and $S_{t i p}$ are the sets of circled and squared nodes,respectively (Figure 4.2):

$$
\begin{aligned}
& S_{\text {tip }}=\left\{K \mid f^{2}\left(x_{K}\right)+g^{2}\left(x_{K}\right) \leqslant r_{\text {tip }}^{2}\right\}, \\
& S_{c r}=\left\{J \mid \min \left(f\left(x_{J}\right)\right) \cdot \max \left(f\left(x_{J}\right)\right) \leqslant 0 \text { and } \max \left(g\left(x_{J}\right)\right) \geqslant 0, J \notin S_{\text {tip }}\right\},
\end{aligned}
$$

where $r_{t i p}$ is predefined radius of a domain around the crack tip to be enriched with a special tip enrichment functions.

Careful analysis of the asymptotic crack tip solution for a Cosserat material [98] reveals that these solutions are spanned by the same functions as those from William's series expansion. Therefore, as in the standard XFEM, it is convenient to enrich the translation 
field in the crack tip domain using the branch functions:

$$
\left\{F_{m}\right\}=\left\{\sqrt{r} \sin \frac{\theta}{2}, \sqrt{r} \cos \frac{\theta}{2}, \sqrt{r} \sin \frac{\theta}{2} \sin \theta, \sqrt{r} \cos \frac{\theta}{2} \sin \theta\right\}
$$

where $(r, \theta)$ are the local polar coordinates with origin at the crack tip.

A similar analysis shows that the crack tip field for the microrotations is spanned by the function:

$$
\left\{F_{m}^{\phi}\right\}=\left\{\sqrt{r} \sin \frac{\theta}{2}\right\}
$$

The Heaviside step function (discontinuous function) is defined in local coordinate system and given by

$$
H(z)=\left\{\begin{array}{cc}
1 & \text { if } z \geqslant 0 \\
0 & \text { otherwise }
\end{array}\right. \text {. }
$$

The jump in displacement (both translation and microrotation) across the crack $\Gamma_{c r}$ is

$$
\begin{gathered}
{[|\mathbf{u}|]=\mathbf{u}^{+}(\mathbf{x})-\mathbf{u}^{-}(\mathbf{x}), \quad \mathbf{x} \in \Gamma_{c r},} \\
{[|\phi|]=\phi^{+}(\mathbf{x})-\phi^{-}(\mathbf{x}), \quad \mathbf{x} \in \Gamma_{c r},}
\end{gathered}
$$

where $\mathbf{u}^{+}$and $\mathbf{u}^{-}\left(\phi^{+}\right.$and $\left.\phi^{-}\right)$are the translations (rotations) above and below the crack, respectively.

Substituting the displacement approximation (4.27) and (4.28) into the (4.34) and (4.35) and simplifying, we have

$$
\begin{aligned}
& {[|\mathbf{u}|]=\sum_{J \in S_{c r}} N_{J} \mathbf{a}_{J}^{u} \text { on } \Gamma_{c r},} \\
& {[|\phi|]=\sum_{J \in S_{c r}} N_{J} \mathbf{a}_{J}^{\phi} \text { on } \Gamma_{c r} .}
\end{aligned}
$$

These equations mean that solution is discontinuous across the crack; $\mathbf{a}_{J}^{u}$ shows the 
opening of the crack.

Following Galerkin approach, the test functions $\mathbf{w}$ and $v$ are taken in the same form as for the translations (4.27) and rotations (4.28), respectively:

$$
\begin{aligned}
\mathbf{w}^{h}(\mathbf{x}) & =\sum_{I \in S} N_{I}(\mathbf{x}) \delta \mathbf{w}_{I}+\sum_{J \in S_{c r}} N_{J}(\mathbf{x})\left[H(f(\mathbf{x}))-H\left(f\left(\mathbf{x}_{J}\right)\right)\right] \delta \mathbf{a}_{J}^{u}+ \\
& +\sum_{K \in S_{\text {tip }}} N_{K}(\mathbf{x}) \sum_{m=1}^{M}\left[F_{m}(r, \theta)-F_{m}\left(r_{n}, \theta_{n}\right)\right] \delta \mathbf{b}_{m K}^{u}, \quad \forall \mathbf{x} \in \Omega, \\
v^{h}(\mathbf{x}) & =\sum_{I \in S} N_{I}^{\phi}(\mathbf{x}) \delta v_{I}+\sum_{J \in S_{c r}} N_{J}^{\phi}(\mathbf{x})\left[H(f(\mathbf{x}))-H\left(f\left(\mathbf{x}_{J}\right)\right)\right] \delta a_{J}^{\phi}+ \\
& +\sum_{K \in S_{t i p}} N_{K}^{\phi}(\mathbf{x})\left[F^{\phi}(r, \theta)-F^{\phi}\left(r_{n}, \theta_{n}\right)\right] \delta b_{K}^{\phi}, \quad \forall \mathbf{x} \in \Omega .
\end{aligned}
$$

Substituting the approximations for the displacements (4.27), (4.28) and the test functions (4.38), (4.39) into the weak forms of the equilibrium equations (4.20) and (4.21) the following discrete equation are obtained:

$$
\left[\begin{array}{llllll}
\mathbf{K}_{11} & \mathbf{K}_{12} & \mathbf{K}_{13} & \mathbf{K}_{14} & \mathbf{K}_{15} & \mathbf{K}_{16} \\
\mathbf{K}_{21} & \mathbf{K}_{22} & \mathbf{K}_{23} & \mathbf{K}_{24} & \mathbf{K}_{25} & \mathbf{K}_{26} \\
\mathbf{K}_{31} & \mathbf{K}_{32} & \mathbf{K}_{33} & \mathbf{K}_{34} & \mathbf{K}_{35} & \mathbf{K}_{36} \\
\mathbf{K}_{41} & \mathbf{K}_{42} & \mathbf{K}_{43} & \mathbf{K}_{44} & \mathbf{K}_{45} & \mathbf{K}_{46} \\
\mathbf{K}_{51} & \mathbf{K}_{52} & \mathbf{K}_{53} & \mathbf{K}_{54} & \mathbf{K}_{55} & \mathbf{K}_{56} \\
\mathbf{K}_{61} & \mathbf{K}_{62} & \mathbf{K}_{63} & \mathbf{K}_{64} & \mathbf{K}_{65} & \mathbf{K}_{66}
\end{array}\right]\left\{\begin{array}{c}
\mathbf{d}^{u} \\
\mathbf{d}^{\phi} \\
\mathbf{a}^{u} \\
\mathbf{a}^{\phi} \\
\mathbf{b}^{u} \\
\mathbf{b}^{\phi}
\end{array}\right\}=\left\{\begin{array}{c}
\mathbf{f}_{\mathbf{1}}^{\text {ext }} \\
\mathbf{f}_{\mathbf{2}}^{\text {ext }} \\
\mathbf{f}_{\mathbf{3}}^{\text {ext }} \\
\mathbf{f}_{4}^{\text {ext }} \\
\mathbf{f}_{\mathbf{5}}^{\text {ext }} \\
\mathbf{f}_{\mathbf{6}}^{\text {ext }}
\end{array}\right\}
$$

where $\mathbf{d}^{u}$ and $\mathbf{d}^{\phi}$ are the standard displacement nodal degrees of freedom that correspond to the translation and rotation, respectively, $\mathbf{a}^{u}$ and $\mathbf{a}^{\phi}$ are the vectors of additional degrees of freedom due to Heaviside enrichment term in the approximations, and $\mathbf{b}^{u}$ and $\mathbf{b}^{\phi}$ are the vectors of the additional degrees of freedom due to the crack tip enrichment. Sub-matrices $\mathbf{K}_{11}, \ldots, \mathbf{K}_{66}$ and $\mathbf{f}_{1}^{\text {ext }}, \ldots, \mathbf{f}_{6}^{\text {ext }}$ are defined in the Appendix A.

Remark 1. $\mathbf{K}$ is not symmetric, unlike in FEM/XFEM classical elasticity. 
Remark 2. The length of vectors $\mathbf{a}^{u}, \mathbf{a}^{\phi}, \mathbf{b}^{u}$ and $\mathbf{b}^{\phi}$ are very small compared to vectors $\mathbf{d}^{u}$ and $\mathbf{d}^{\phi}$, so XFEM does not significantly increase the cost of solving (4.40).

\subsection{Numerical integration}

Several aspects that are crucial in order to obtain accurate results and optimal convergence rates are listed below [117]:

- Numerical integration is an important issue in the XFEM. The proper integration rules in the elements cut by a crack and containing a crack tip should be considered.

- The fixed enrichment area around a crack tip (geometrical enrichment) is crucial to obtain optimal convergence rates. It allows to get twice better energy convergence rate than in case of the topological enrichment, when only particular number of nodes layers are enriched.

- Blending elements allows for the smooth transition between enriched and unenriched subdomains by using the special weight function with compact support.

The considerable amount of works on the research of numerical integration for the XFEM is presented in [118]. There are two difficulties for the integration in the XFEM. First, in elements cut by the interface, the shape functions may be discontinuous. Second, if the element contains a crack tip, the enriching functions and their gradients are singular. That means that the integration scheme depends on whether the element is enriched and on the type of enrichment. The integration of unenriched elements is done in the standard way using Gauss quadrature of the required order. If the element is fully cut by the interface, i.e. enriched by the Heaviside step function, the sub-integration is employed. The element is divided into triangles in such a way that the crack surface is aligned with the edges of two triangles [69]. In the present research, for both unenriched elements and elements enriched with the Heaviside function the Gauss quadrature of order 2 is employed. The subintegration is used in the latter case. 
If the element contains the crack tip, the element is divided into the several sub-triangles having one vertex at the crack tip [119]. In each triangle the higher order Gauss quadrature is used. This creates the concentration of integration points near the node of the triangle located at the crack tip. This technique with the Gauss quadrature of order 15 is employed in the present research (Figure 4.4).

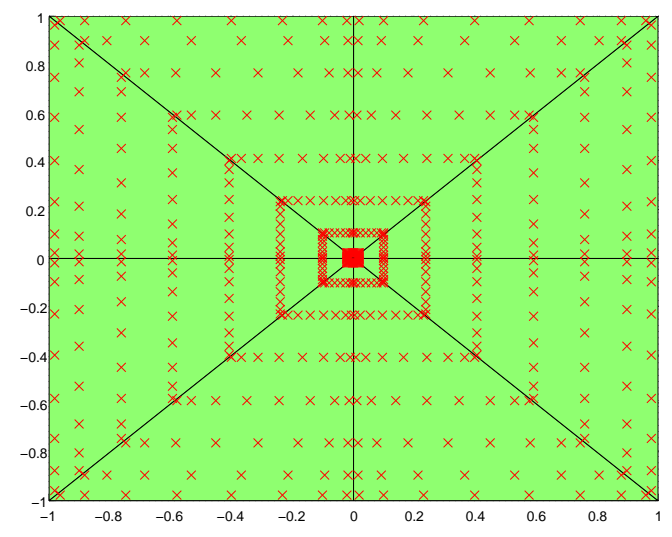

Figure 4.4: The representation of the Gauss quadrature rule in the element containing crack tip.

Considering the branch function zone enrichment, there are two ways to define the branch functions enrichment zone around the crack tip: the geometrical enrichment and the topological enrichment. First approach defines the enriched nodes within the constant radius. The second approach defines the enriched nodes in the particular number of nodes layers around the crack tip. To get optimal behaviour in energy norm for the XFEM, the geometrical enrichment should be considered. In the case of the geometrical enrichment, the branch function enrichment area is independent of the mesh size. Two types of crack tip zone enrichment are illustrated in Figure 4.5-4.6.

Another important issue in the XFEM is the blending elements. The blending elements are those that have some of the nodes enriched and some of them unenriched [116]. The standard enrichment can add parasitic terms to the approximation space of the blending elements, i.e. the reproducing property of the partition of unity is lost. No difficulties occurs in the blending of the shifted Heaviside enrichment, because the enrichment is 
only considered for the elements cut by crack. However, for the tip enrichment zone, the blending might play an important role to reduce the error value.

Concluding, the use of proper integration rules, depending on the type of elements is crucial for the accuracy of the solution and obtaining optimal convergence rates. The differences in convergence rates of the finite element method, extended finite element method with topological and geometrical tip enrichments, and without tip enrichment are shown further in Chapter "Numerical Examples".

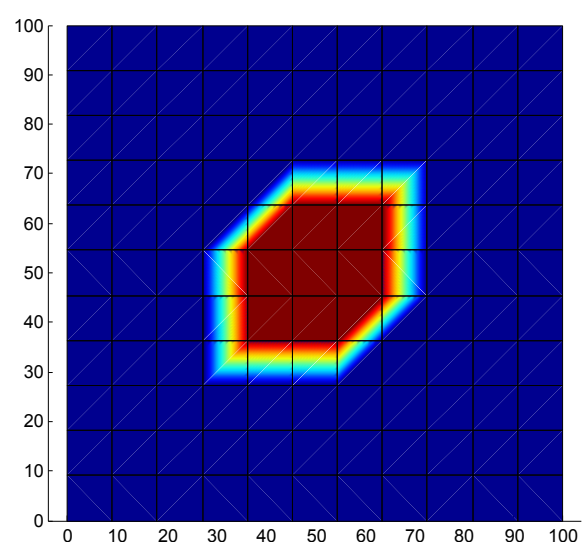

(a)

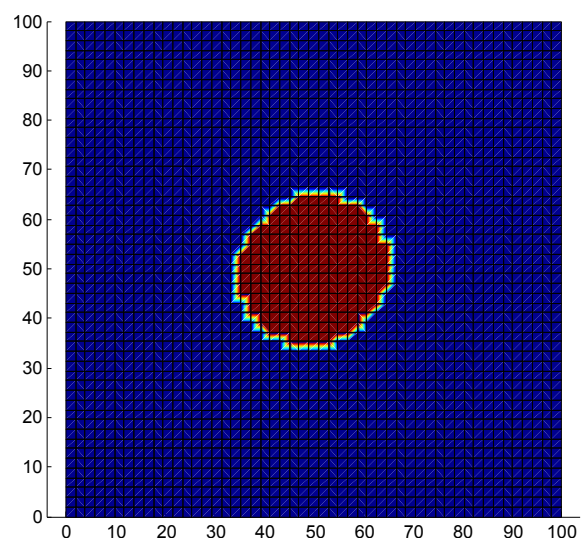

(b)

Figure 4.5: Selected elements for the geometrical enrichment with the number of elements: (a) $11 \times 11$, (b) $51 \times 51$. 


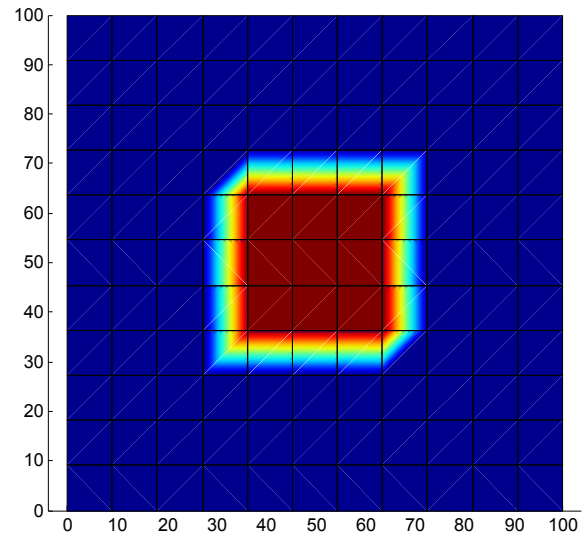

(a)

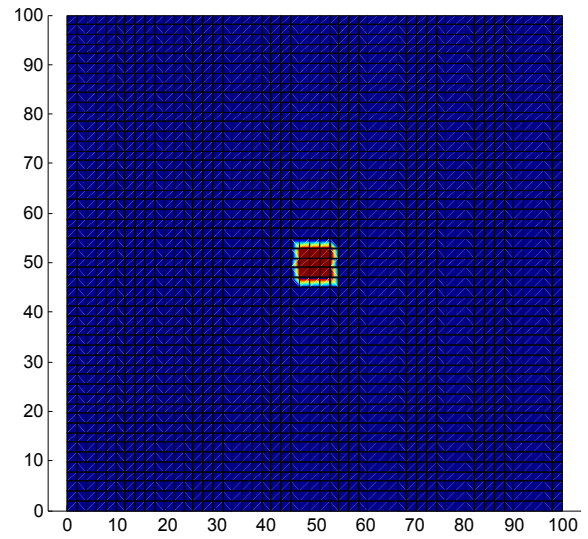

(b)

Figure 4.6: Selected elements for the topological enrichment with the number of elements: (a) $11 \times 11$, (b) $51 \times 51$. 


\subsection{Crack opening model}

The step-by-step algorithm of the XFEM modelling of a crack is presented below.

1. Defining the model Define the boundary of the physical model, the boundary and loading condition, the material model.

2. Front tracking Define the crack geometry (front) using level set method.

3. Enrichment detection Define enrichment types and assign associated nodes (nodes enriched by the jump function, nodes enriched by the tip enrichment functions, etc.).

4. Solving Evaluate both the enrichment functions and its derivatives. Build stiffness matrix and force vector. Solve the system of equations $\boldsymbol{d}=\boldsymbol{K} \backslash \boldsymbol{F}$.

5. Post-processing Calculate J-integral. 


\subsection{Computation scheme of the J-integral evaluation}

1. Load FEM/XFEM solution and the crack information to the Postprocessor.

2. Define the contour radius and location, number of sectors.

3. For $\mathrm{n}=1$ to number of sector, calculate Jacobian and define the Gauss quadrature order.

4. For $\mathrm{q}=1$ to number of quadrature points, obtain the global coordinates of the quadrature point, define the element of the mesh containing the point, obtain the vector of element nodal displacements from the displacement vector, define a normal to a contour at a point; calculate stress, strain, curvature components, derivatives of the displacements, and strain energy at the point; calculate J-integral at a point and sum the value to the global J-integral.

5. Go to step 4.

6. Go to step 3 . 


\section{Chapter 5}

\section{Numerical Examples}

In this chapter, the numerical examples of cracks under the assumption of the plane strain in two-dimensional Cosserat elastic medium are presented. The series of patch tests and convergence studies in the first three examples verify the method and demonstrate the convergence properties of the FEM and the XFEM solutions of the edge crack problem in a Cosserat elastic medium. The convergence properties of the XFEM solution of the edge crack problem under tension and shear loading are demonstrated. The forth example presents the verification of the contour J-integral calculation. In the fifth example a comparison of the J-integral for the Cosserat elasticity and the classical elasticity is presented and discussed.

\subsection{Introduction}

As stated in [98], the Cosserat parameter $\alpha$ varies from 0 (classical linear elasticity) to $\infty$ (couple stress linear elasticity) and has a huge impact on the asymmetrical properties of the stress and strain tensors. Several problems with $\alpha \neq 0$ were solved to demonstrate the effectiveness of the developed XFEM/Cosserat method.

In order to perform convergence studies in the following examples, the normalized energy norms are employed, see Section 3.4.2 for details. For the following examples, the 
sequence of the employed mesh sizes is $10 \times 10,20 \times 20,30 \times 30,40 \times 40,60 \times 60,80 \times 80$ elements.

\subsection{Convergence study of the FEM/Cosserat solution for the domain without a crack with $\alpha \neq 0$}

Consider a square plate of size $L \times L$ as shown in Figure 5.1, where $L=100 \mathrm{~mm}$. Along the boundary of the domain, displacements are prescribed using the following functions:

$$
\begin{aligned}
& u_{x}=3 x^{2}+8 y^{2}+10 x y \\
& u_{y}=9 x^{2}+20 y^{2}+x y \\
& \phi=50 \cos \left(\frac{2 \pi x}{10}\right) \sin \left(\frac{2 \pi y}{10}\right) .
\end{aligned}
$$

The material properties are: the Young's modulus $E=10000 \mathrm{MPa}$, the Poisson's ratio $\nu=0.3, \lambda=5796 \mathrm{~N} / \mathrm{mm}^{2}$, the coupling number $N=0.5$, the elastic constants $\mu=3846 \mathrm{~N} / \mathrm{mm}^{2}, \kappa=81 \mathrm{~N} / \mathrm{mm}^{2}, \gamma=1000 \mathrm{~N}, \beta=0 \mathrm{~N}$, and $\alpha=0,100,1000 \mathrm{~N} / \mathrm{mm}^{2}$.

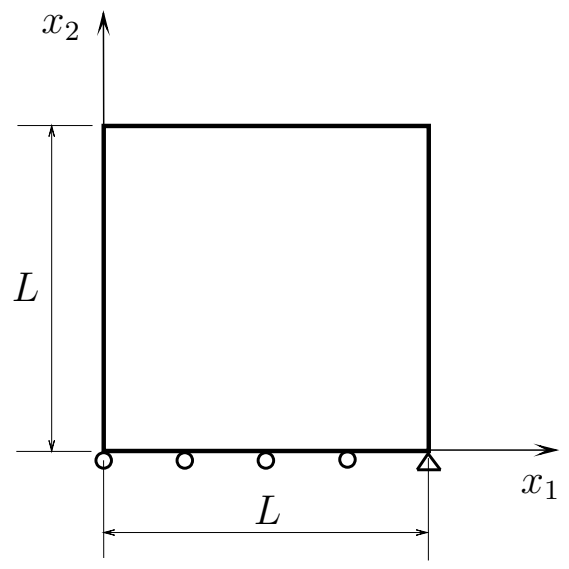

Figure 5.1: The square domain without a crack. 
As shown in Figure 5.2, the obtained convergence rates for various $\alpha$ are in order of $\approx 1$ in the energy norm, which is the optimal convergence rates for the domain without a crack. This example shows that the FEM solution of the Cosserat problem with $\alpha \neq 0$ is correct and could be used as the exact solution for the following convergence test of the XFEM problem.

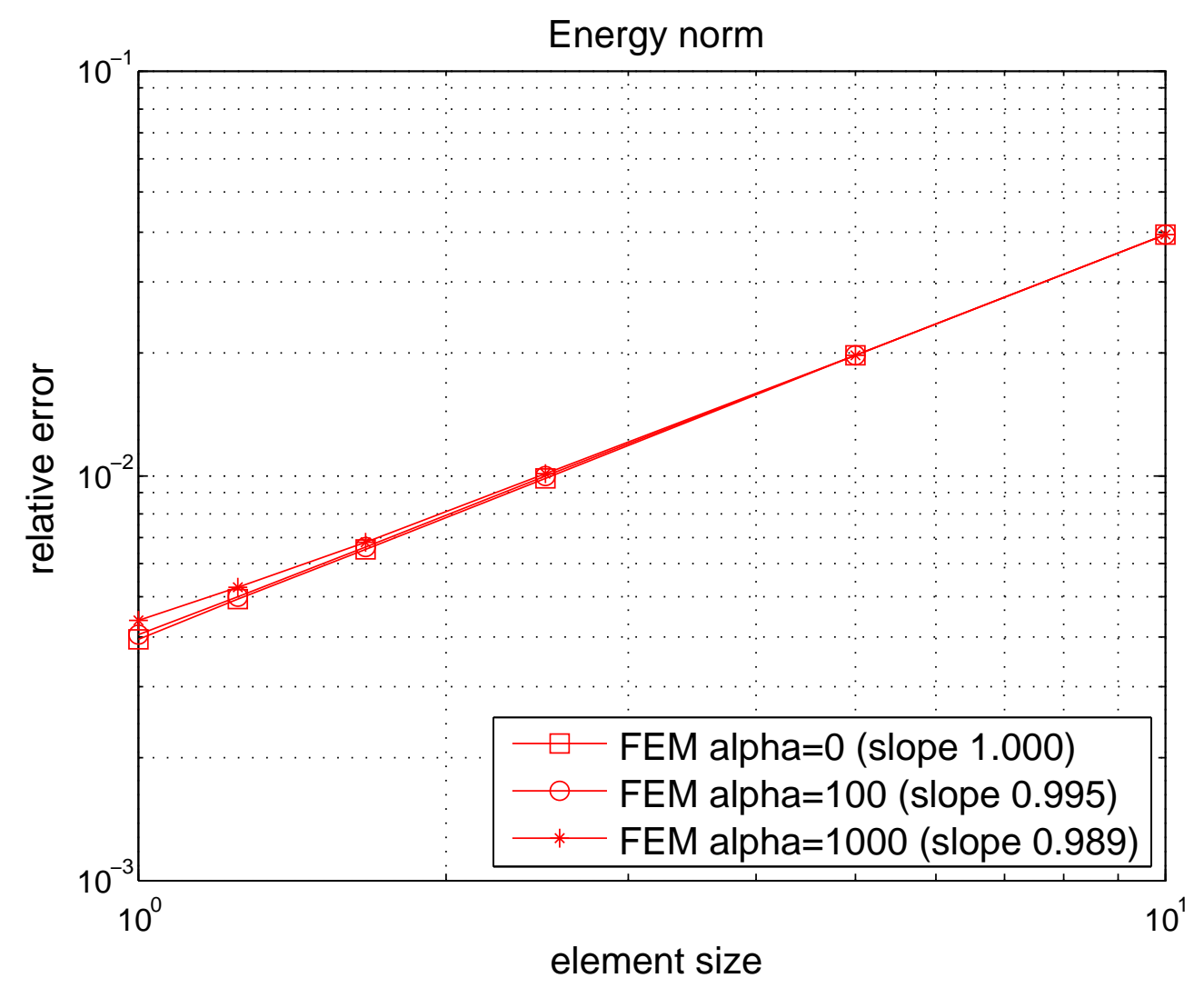

Figure 5.2: Convergence rates of the FEM solutions for the $\alpha=0,100,1000$. 


\subsection{Convergence study of the XFEM/Cosserat solu- tion of the edge crack problem in the domain with the prescribed analytical solution on the bound- aries with $\alpha=0$}

A square plate of size $L \times L$ is considered with an edge crack of length $a$, where $L=100 \mathrm{~mm}$ and $a=L / 2$, as shown in Figure 5.3. Along the boundary of the domain, displacements are prescribed using (2.13) and (2.14) such that the known analytical solution of a near tip crack field is the exact in the entire domain. For numerical computation in this example, Mode I crack is assumed, i.e. $K_{I}=205$ and $K_{I I}=0 \mathrm{Nmm}^{\frac{3}{2}}$. The crack surface is traction-free.

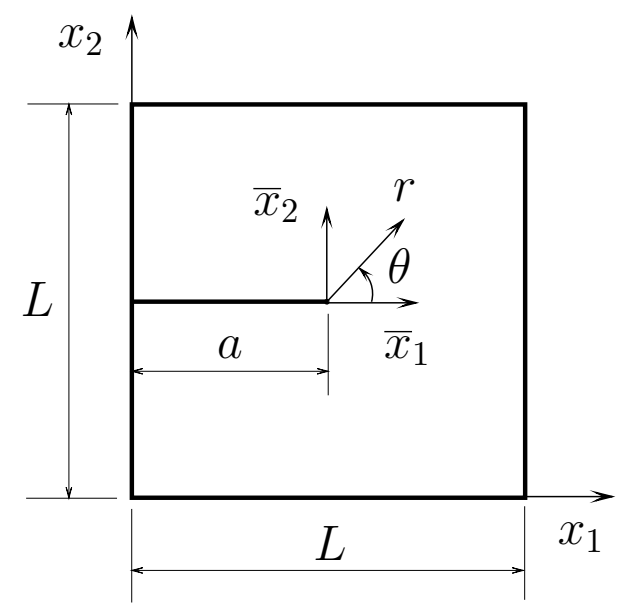

Figure 5.3: Edge crack in a finite domain.

The material properties are taken to be as follows: $E=10000 M P a, \nu=0.3, \lambda=$ $5796 \mathrm{~N} / \mathrm{mm}^{2}$, the coupling number $N=0.5$, the elastic constants $\mu=3846 \mathrm{~N} / \mathrm{mm}^{2}$, $\kappa=81 \mathrm{~N} / \mathrm{mm}^{2}, \gamma=1000 \mathrm{~N}, \beta=0 \mathrm{~N}$, and $\alpha=0 \mathrm{~N} / \mathrm{mm}^{2}$.

First, the problem was solved by the FEM, the XFEM with topological tip enrichment, and the XFEM with geometrical tip enrichment. In the problem, solved by the standard 
FEM, the crack surface is aligned with the element edges, and the crack tip is located at the node. In the XFEM computation discontinuity is presented independently of the mesh. The results of the convergence study of the Mode I edge crack in the square domain are presented in Figure 5.4. The sub-optimal convergence rates of $\approx 0.5$ in energy norm are obtained. The convergence rates of the FEM solution are reduced due to the presence of singularity in the vicinity of the crack tip. The problem solved by the XFEM technique with the geometrical tip enrichment shows optimal convergence rates of $\approx 1$ in energy norm. The convergence of the XFEM solution with the topological tip enrichment is studied, as well. The suboptimal convergence rate of $\approx 0.5$ in energy norm is obtained by that method, as expected. The crucial issues for obtaining the optimal convergence rates are the use of special integration methods in the crack-tip element and the element cut by crack, branch function enrichment of the fixed area around the crack tip (so called, geometrical enrichment) and the specific treatment of the blending elements. All of the above mentioned techniques are described in detail in Section 4.4 "Numerical integration".

Analogous convergence study was performed for the Mode II edge crack problem, i.e. $K_{I}=0$ and $K_{I I}=200 \mathrm{Nmm}^{\frac{3}{2}}$. Similarly to the presented results of the Mode I edge crack, the optimal convergence rates are obtained by the XFEM with the geometrical tip enrichment. 


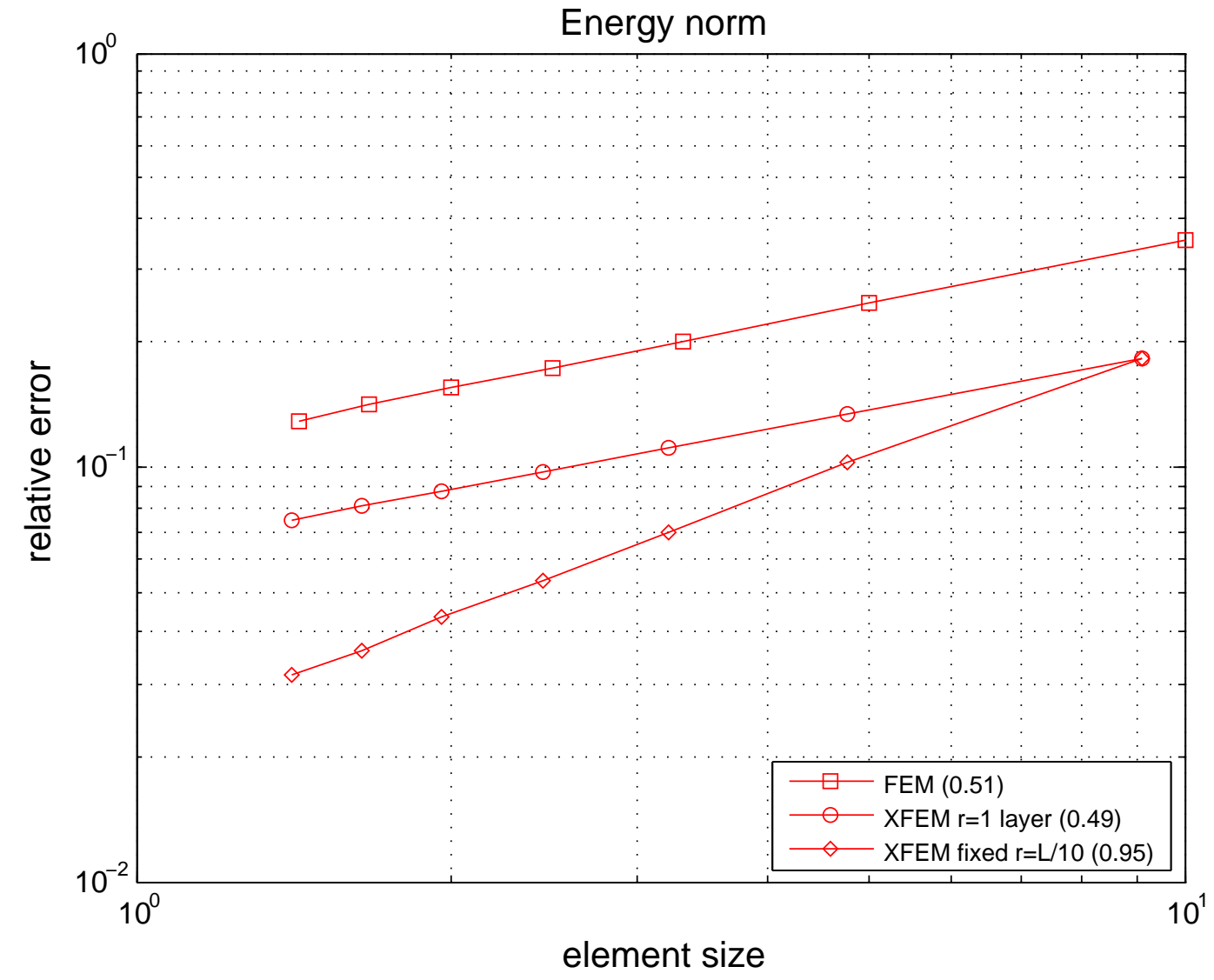

Figure 5.4: Convergence study of the Mode I edge crack problem in a Cosserat medium with $\alpha=0$. 
Second, the tip enrichment types were studied to find the optimal way to asymptotically enrich the nodes in the vicinity of the crack tip. Considering the topological tip enrichment, Figure 5.5 shows the dependence of the error values on the number of the enriched with the branch functions nodes layers around the crack tip. With the increasing number of enriched nodes layers, the error value is getting smaller. This is explained by the fact that larger area is enriched with the known asymptotic solution.

Considering the geometrical tip enrichment, the studies of the convergence rates depending on the asymptotic enrichment radius are presented in the Figure 5.6. Although, the error value is getting smaller for the bigger tip radius enrichment, this causes the dramatically increased computational time. With the increased radius of tip enrichment, the number of enriched nodes and elements with higher order Gauss quadrature are getting larger. On the other hand, the radius of tip enrichment zone equal or smaller than $L / 15$ causes the less accurate solution. Taking into account all of these observations, the optimal enrichment radius of $L / 10$ is adopted for the further modelling. 


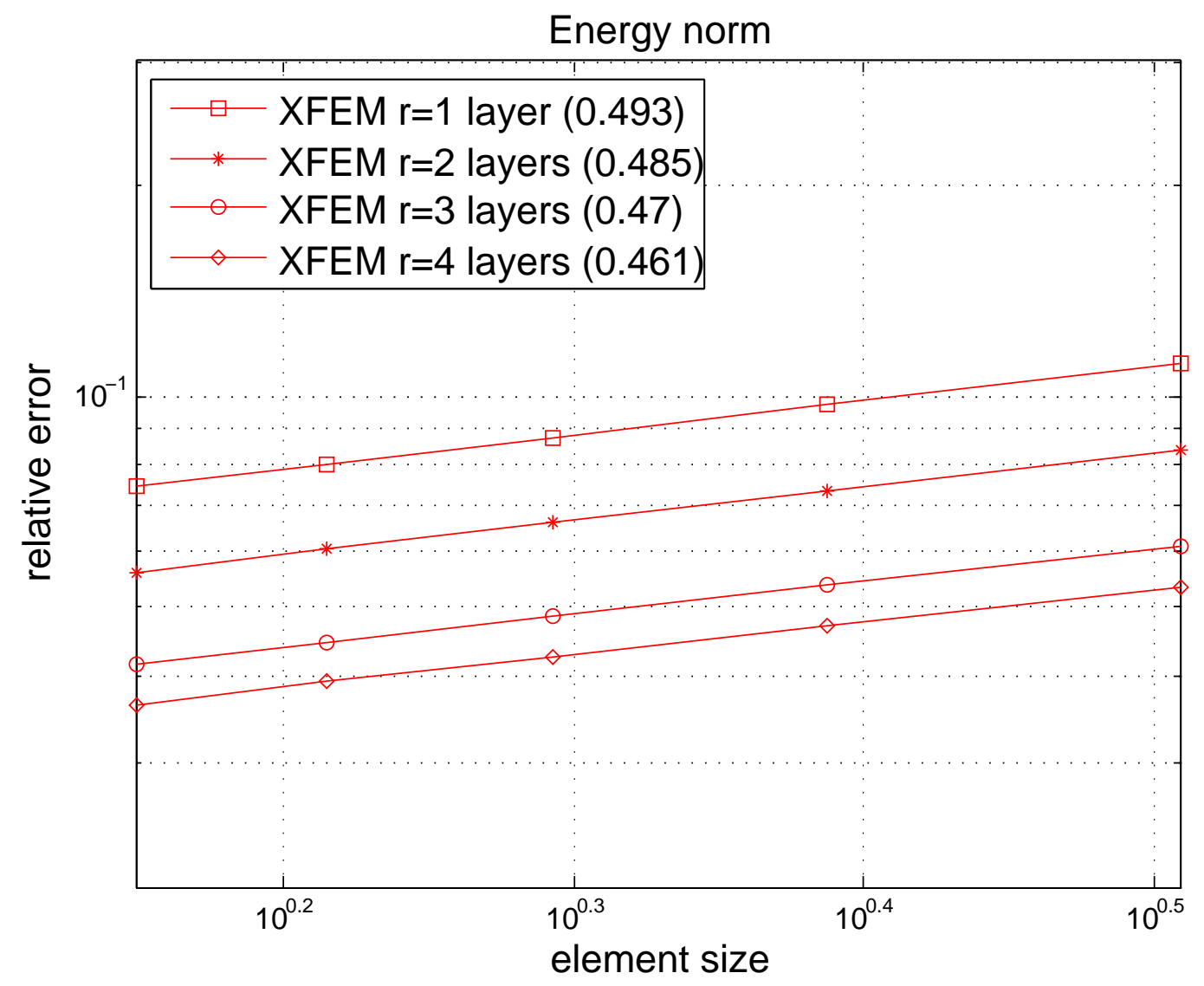

Figure 5.5: Convergence study for the coupled Mode I edge crack case solved by XFEM with topological tip enrichment with varying number of enriched nodes layers from 1 to 4. 


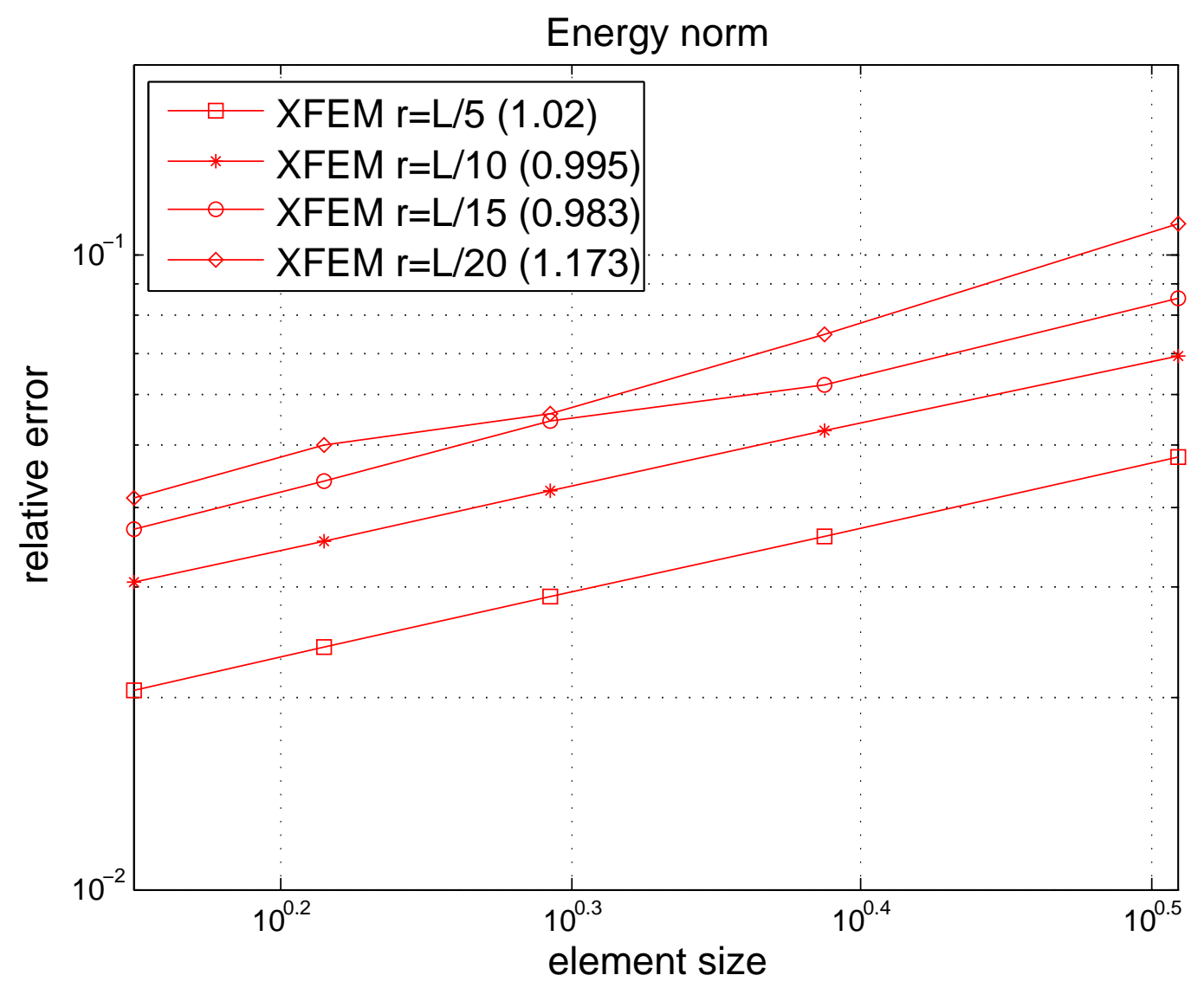

Figure 5.6: Convergence study for the coupled Mode I edge crack case solved by XFEM with geometrical tip enrichment with varying radius from $L / 5$ to $L / 20$. 


\subsection{Convergence study of the XFEM/Cosserat solu- tion of the edge crack problem with $\alpha \neq 0$}

\subsubsection{Mode I edge crack}

A square plate of size $L \times L$ is considered with an edge crack of length $a$ as shown in Figure 5.7, where $L=100 \mathrm{~mm}$ and $a=L / 2$. The domain is fixed at the bottom, and the displacement $u_{2}=5 \mathrm{~mm}$ in $x_{2}$ direction is prescribed along the top edge. The microrotations $\phi=0$ are prescribed along the bottom and top edges of the domain, which allows for the microrotations to emerge around the crack tip. The crack surface is traction-free. The plain strain condition is assumed. The material properties are: the Young's modulus $E=10000 \mathrm{MPa}$, the Poisson's ratio $\nu=0.3, \lambda=5796 \mathrm{~N} / \mathrm{mm}^{2}$, the elastic constants $\mu=3846 \mathrm{~N} / \mathrm{mm}^{2}, \kappa=81 \mathrm{~N} / \mathrm{mm}^{2}, \gamma=1000 \mathrm{~N}, \beta=0 \mathrm{~N}$. As it was mentioned above, $\alpha=0$ reduces the problem to the one of the classical elasticity, and $\alpha \rightarrow \infty$ implies the couple stress elasticity problem. For this example, $\alpha$ was taken to be $10^{3} \mathrm{~N} / \mathrm{mm}^{2}$.

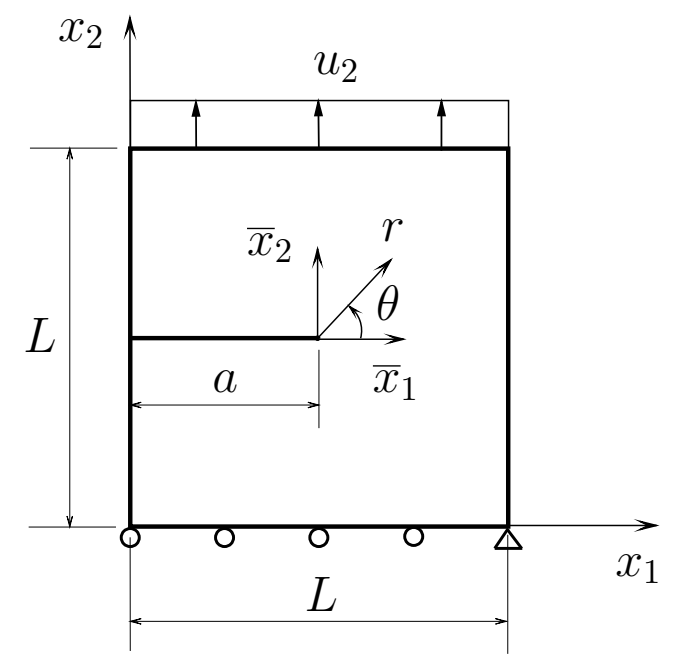

Figure 5.7: The domain with an edge crack under tension.

In order to perform the convergence study for the Mode I and II edge crack problems, the normalized energy norm is employed as a convergence criterion. The employed mesh 
sizes are $11 \times 11,21 \times 21,31 \times 31,41 \times 41,51 \times 51,71 \times 71$ elements. The solution of the addressed Mode I edge crack problem by the Finite Element Method with the mesh of 40,000 elements was taken to be the exact solution. Two types of the tip enrichment zones were considered for the convergence study of the XFEM: the topological tip enrichment of the one layer of nodes around the crack tip, the geometrical tip enrichment of the size $L / 10$. Figure 5.8 shows three convergence curves and the corresponding convergence rates for the Mode I edge crack solution in a Cosserat medium.

As expected, the optimal convergence rate of $\approx 1$ in the energy norm for the XFEM with geometrical enrichment is obtained. The suboptimal convergence rates of $\approx 0.5$ in energy norm are obtained for the XFEM solutions with topological tip enrichment and for the FEM solution. 


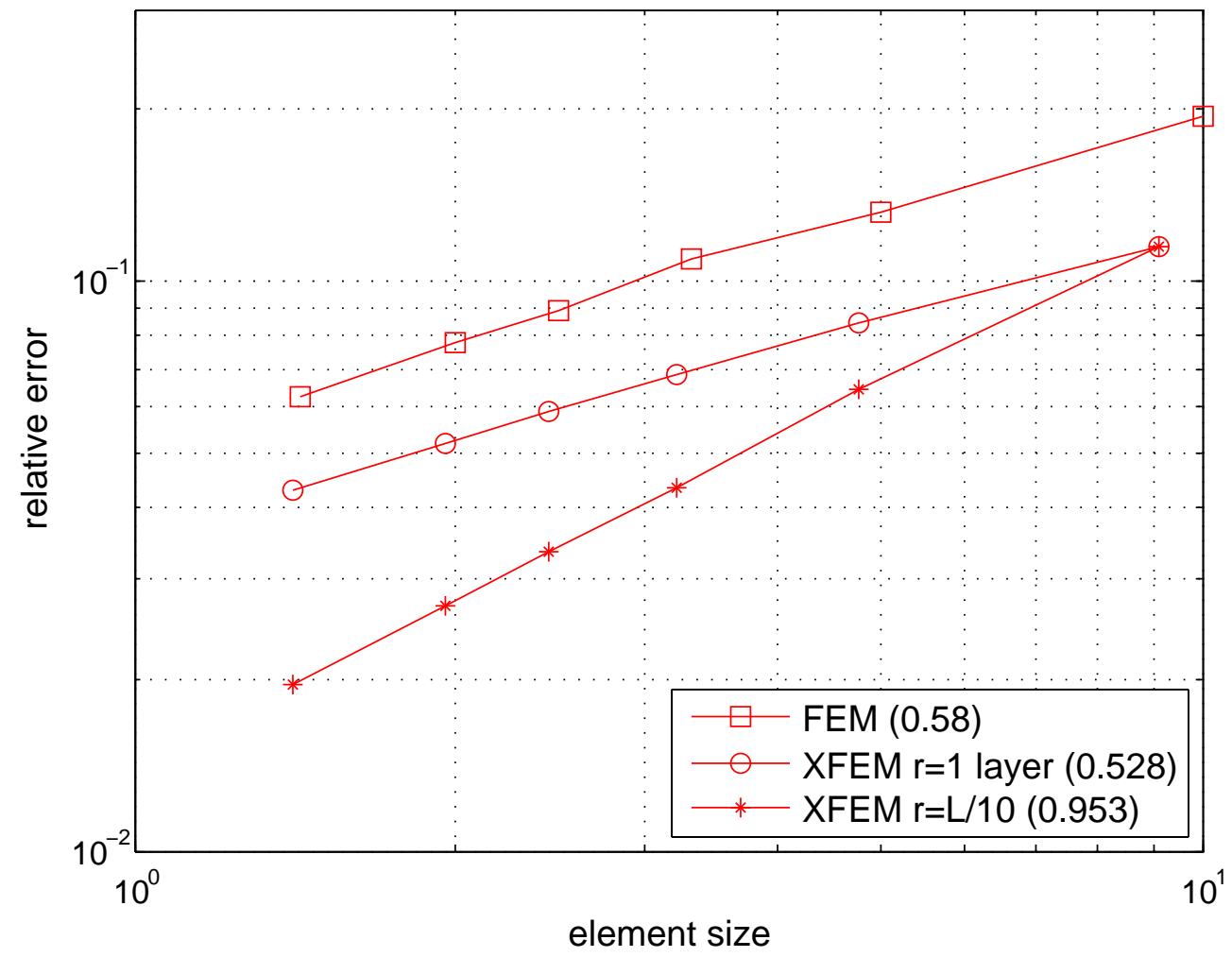

Figure 5.8: Convergence rates of the XFEM solutions of the edge crack problem under the tension loading with $\alpha=1000$. 


\subsubsection{Mode II edge crack}

In the second example, Mode II edge crack problem is studied. Consider a square plate of size $L \times L$ with an edge crack of length $a$, as shown in Figure 5.9. The length of the plate is $L=100 \mathrm{~mm}$, and the crack size is $a=L / 2$. The domain is fixed at the bottom. The displacement $u_{1}=5 \mathrm{~mm}$ in $x_{1}$ direction is prescribed along the top edge. The microrotations are prescribed to be $\phi=0$ along the bottom and top edges of the domain. The crack surface is traction-free. The plain strain condition is assumed. The material properties are: the Young's modulus $E=10000 \mathrm{MPa}$, the Poisson's ratio $\nu=0.3$, $\lambda=5796 \mathrm{~N} / \mathrm{mm}^{2}$, the elastic constants $\mu=3846 \mathrm{~N} / \mathrm{mm}^{2}, \kappa=81 \mathrm{~N} / \mathrm{mm}^{2}, \gamma=1000 \mathrm{~N}$, $\beta=0 N$.

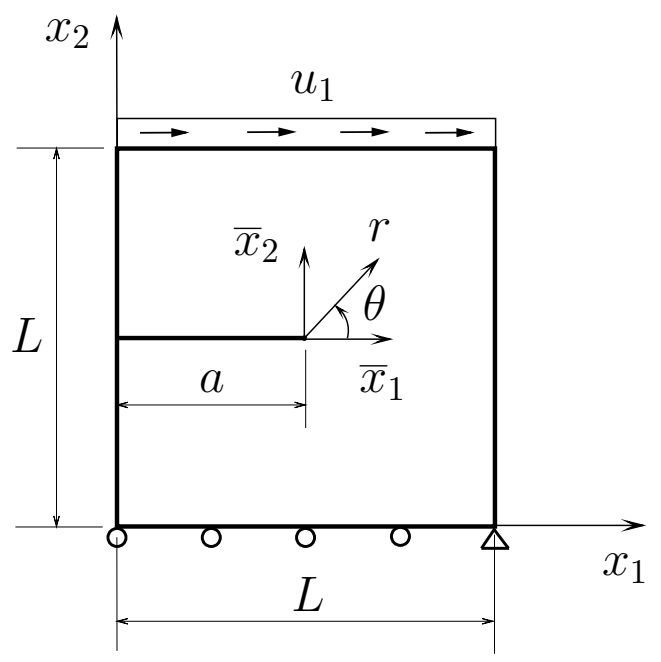

Figure 5.9: The square domain with an edge crack under shear loading.

The convergence rates are shown in Figure 5.10. For the Mode II edge crack problem in a Cosserat medium, the optimal convergence rate of $\approx 1$ in energy norm was obtained for the XFEM solution with the geometrical tip enrichment, and the suboptimal convergence rate of $\approx 0.5$ in energy norm was obtained for the XFEM solution with the topological tip enrichment.

For both Mode I and II edge crack problems, the slight decrease in the convergence rates from the optimal values is observed, but expected. In order to get higher convergence 


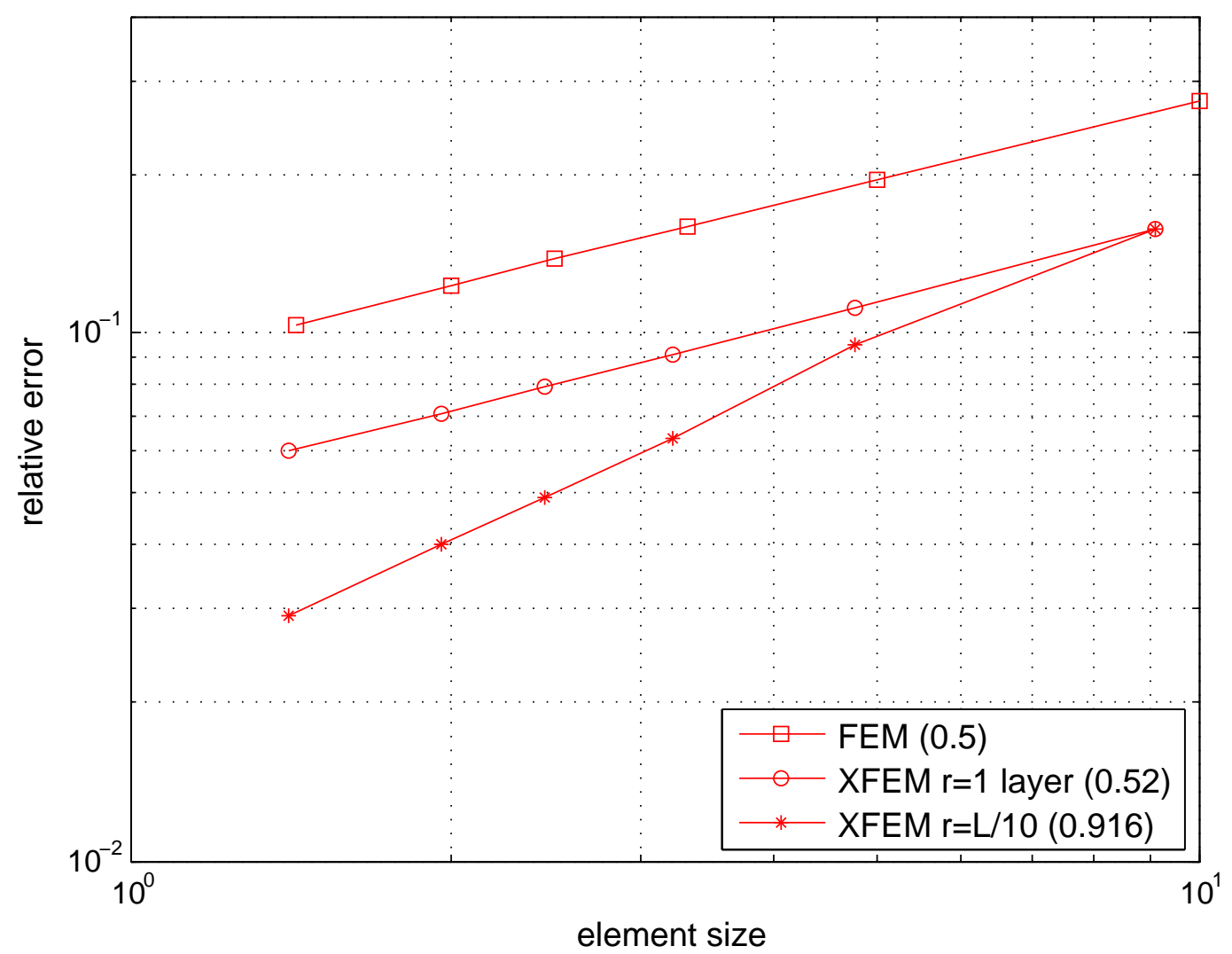

Figure 5.10: Convergence rates of the FEM solutions of the edge crack problem under the shear loading for the $\alpha=1000$.

rates, the very fine mesh should be considered for the exact FEM solution. Although, the computational time will increase drastically.

Concluding, the results of the convergence studies for the cracks of Modes I and II in the Cosserat medium allows us to rely on the developed XFEM model with confidence. 


\subsection{Calculation of the J-integral in a Cosserat medium}

\subsubsection{Verification of the J-integral calculation}

Consider a square plate of size $L \times L$, with an edge crack of length $a, L=100 \mathrm{~mm}$ and $a=L / 2$, as shown in Figure 5.11. The known asymptotic displacement field solutions (2.13-2.14) are prescribed on the boundaries of the domain. The crack surface is tractionfree. The material properties are: the Young's modulus $E=10000 \mathrm{MPa}$, the Poisson's ratio $\nu=0.3, \lambda=5796 \mathrm{~N} / \mathrm{mm}^{2}$, the coupling number $N=0.5$, the elastic constants $\mu=3846 \mathrm{~N} / \mathrm{mm}^{2}, \kappa=81 \mathrm{~N} / \mathrm{mm}^{2}, \gamma=1000 \mathrm{~N}, \beta=0 \mathrm{~N}$, and $\alpha=0 \mathrm{~N} / \mathrm{mm}^{2}$.

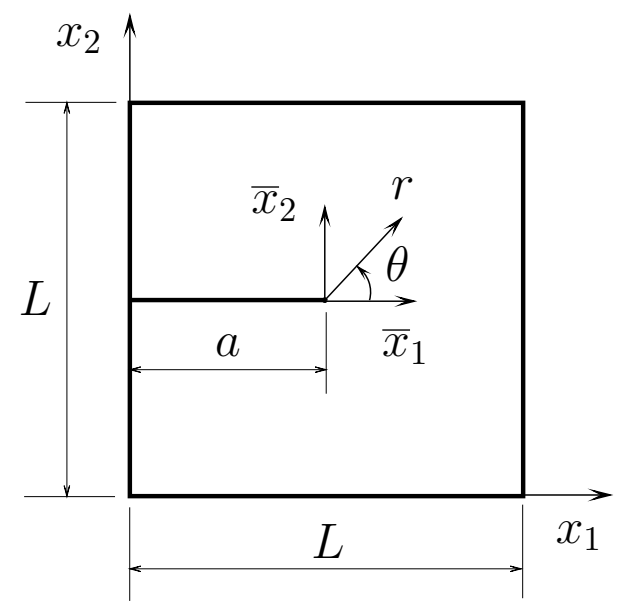

Figure 5.11: The domain with an edge crack. The asymptotic field solutions are prescribed on the boundaries.

First, the path-independence of the J-integral is studied. The path-independence is the important characteristic of the contour integral. In this research, the contour of the J-integral is assumed to be a circle with its center coinciding with the crack tip. Figure 5.12 shows that the calculated J-integral is converging and path-independent. The case with geometrical tip enrichment of radius $L / 10$ gives more accurate values and smooth behaviour of the J-integral curve. The kink at the $R=10 \mathrm{~mm}$ corresponds to the end of the tip enrichment radius 10. As well, the slight disinclination of the J-integral values is observed 
for the radius $\leq 20 \mathrm{~mm}$, where the higher values of stresses and strains in the vicinity of the crack tip are presented. For the purpose of consistency, the J-integral is calculated at the radius $\geq 20 \mathrm{~mm}$ in the further tests. As well, the J-integral is zero on the contour without the discontinuity, which is satisfied by the presented numerical calculation.

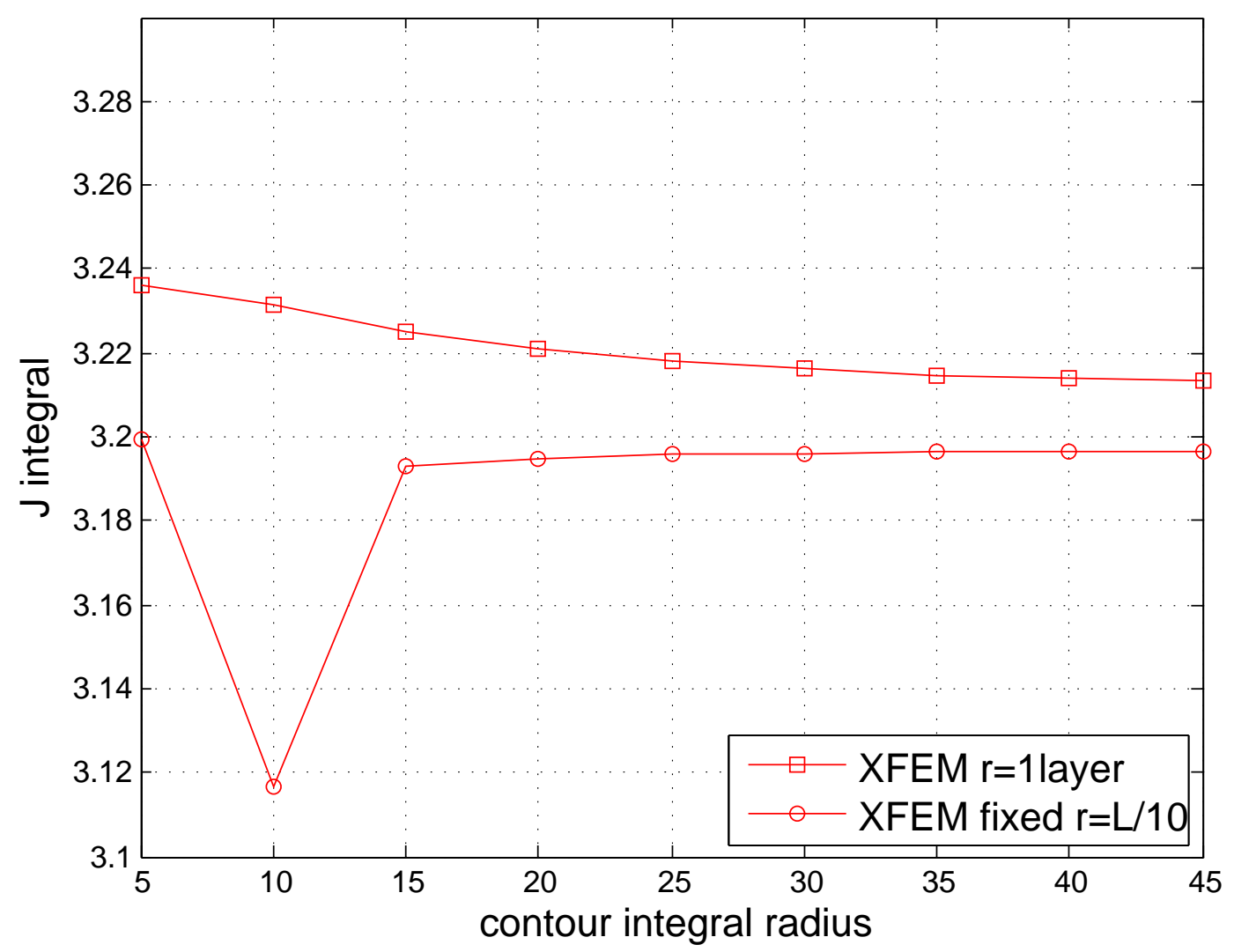

Figure 5.12: The representation of the J-integral dependence on the contour radius. 
Second test compares the numerically calculated for the reduced to the classical case J-integral with the one obtained analytically. In the classical theory of elasticity, J-integral for the Mode I crack is calculated in the following way:

$$
J=\frac{K_{I}^{2}}{E^{*}},
$$

where $E^{*}=\frac{E}{1-\nu^{2}}$ for the plane strain case and $E^{*}=E$ for the plane stress case. Only the Cosserat model, reduced to the classical, could be tested this way, as there is no known relationship between $L_{I}$ and J. The numerical values of the J-integral was found to be less than $1 \%$ different from the analytically obtained values.

In addition, the J-integral can be approximated as the change in the energy of the domain due to crack length increment, i.e $\triangle U / \triangle a$. Considering a mesh of $101 \times 101$ elements with the crack increment $\triangle a=3 \mathrm{~mm}$, for the Mode I crack case the deviation from the analytical value was of $4 \%$, and for the Mode II crack case the deviation was of $5 \%$. Taking into account the fact that this approximation is valid for the crack increment approaching zero $\triangle a \rightarrow 0$, the obtained results are accurate enough to consider the calculation of the J-integral in the Cosserat elastic medium to be correct.

Forth, the dependence of the J-integral from $K_{I}$ is studied and shown in Figure 5.13. The slope of the line is 2 , and the $y$-intercept is $7.58 \times 10^{-5}$, which corresponds to the value of $K_{I}^{2}\left(1-\nu^{2}\right)$, as expected from the power law (5.4) for the case of plane strain.

The obtained results verify the most important characteristics of the contour J-integral, which allow us to proceed to the study of the energy release rate for the Cosserat medium. 


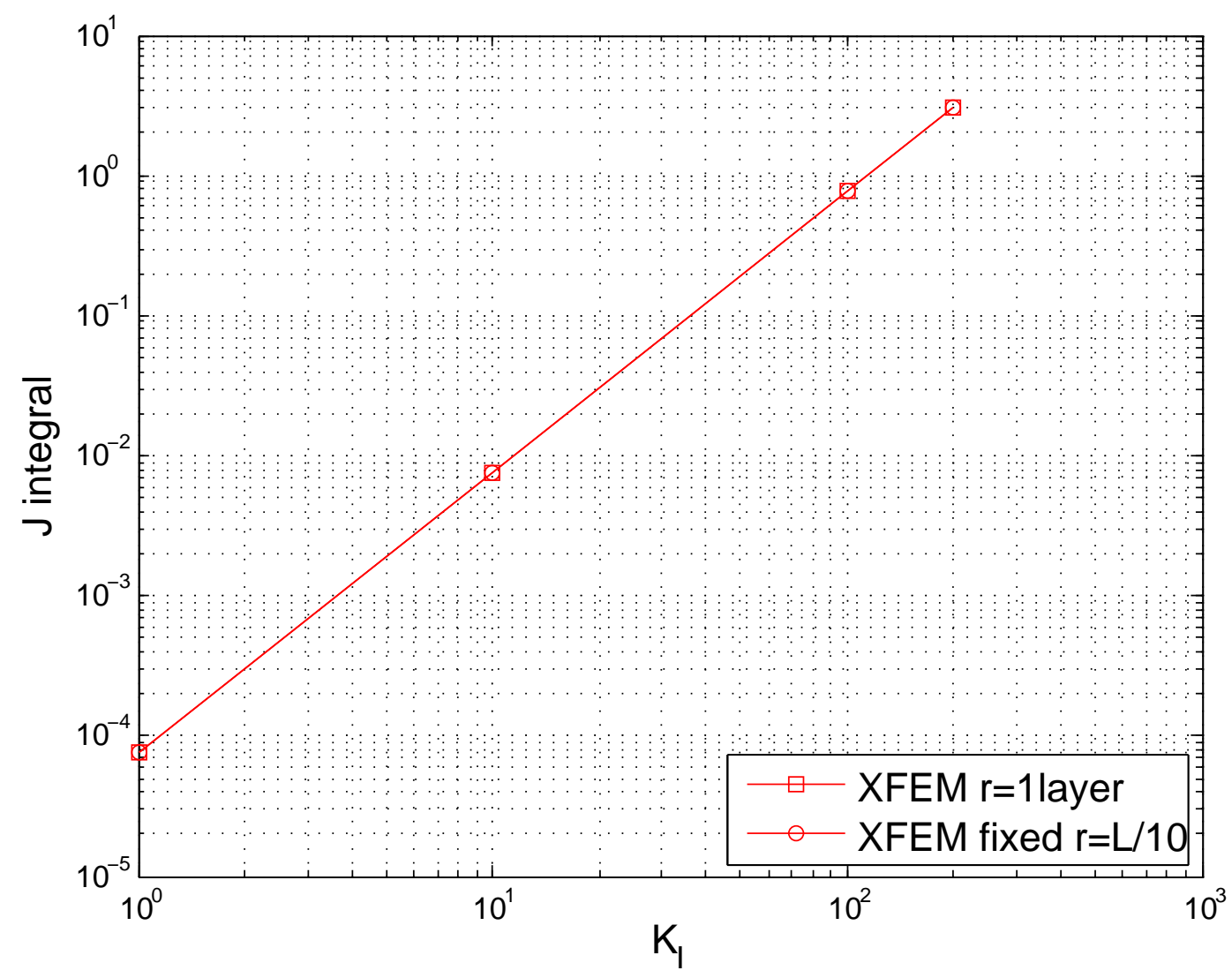

Figure 5.13: The representation of dependence of J-integral from $K_{I}$ with $L_{I}=0$. 


\subsubsection{Comparison of the J-integral for the Cosserat elastic medium and classical elastic medium}

Consider the domain illustrated in Figure 5.14. A plate of height $L$ and width $W$ contains an edge crack of length $a$, where $L / W=2, W=100 \mathrm{~mm}$ and $a$ has varying value. The bottom edge of the domain is fixed. The stress $\sigma=3 \mathrm{MPa}$ is applied at the top edge of the domain. The Young's modulus is $E=10000 \mathrm{MPa}$, the Poisson's ratio is $\nu=0.3$, and the material constants are: $\lambda=5796 \mathrm{~N} / \mathrm{mm}^{2}, \mu=3846 \mathrm{~N} / \mathrm{mm}^{2}, \kappa=81 \mathrm{~N} / \mathrm{mm}^{2}$, $\gamma=1000 N, \beta=0 N$.

In this example, the effect of the crack size on the J-integral is analysed. Several cases are considered depending on the values of the Cosserat elastic constant $\alpha$, which influences the level of asymmetry of the stress and strain tensors. For this study, parameter $\alpha$ is chosen to be $\alpha=10^{-1}, 10^{2}, 10^{3}, 10^{4} \mathrm{~N} / \mathrm{mm}^{2}$, which is attributed to the Cosserat elastic theory. The mesh size is $101 \times 101$ elements. The elements to be enriched by the asymptotic field are chosen within the geometrical tip enrichment zone of size $L / 10$.

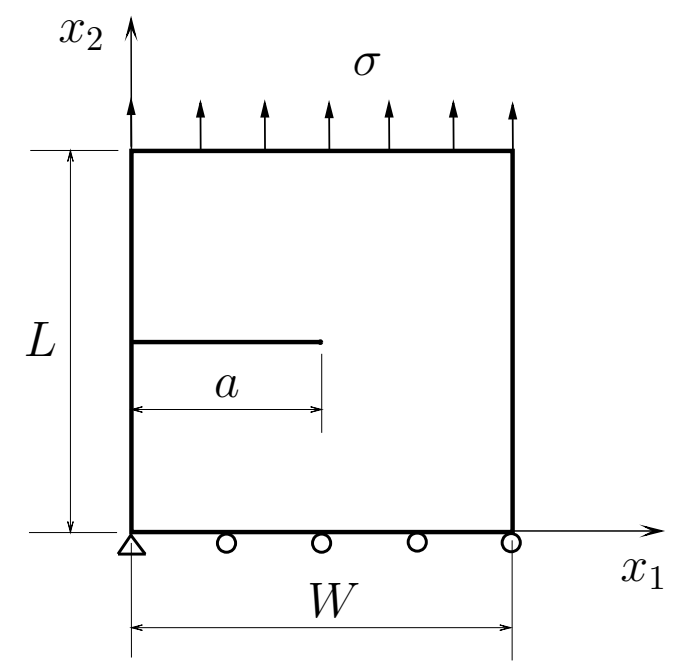

Figure 5.14: The domain with an edge crack under shear.

The calculated J-integral for the Cosserat elasticity is compared to the one of the classical elasticity. The exact stress intensity factor solution of the addressed problem for 
the classical elasticity is given in [120]:

$$
K_{I}=C \sigma \sqrt{a \pi}
$$

where $C$ is a geometry correction factor.

For $a / W \leq 0.6$, the factor $C$ is defined as

$$
C=1.12-0.231\left(\frac{a}{W}\right)+10.55\left(\frac{a}{W}\right)^{2}-21.72\left(\frac{a}{W}\right)^{3}+30.39\left(\frac{a}{W}\right)^{4} .
$$

The reference J-integral for the Mode I crack under the plane strain condition is obtained from the following equation:

$$
J_{I}^{r e f}=\frac{K_{I}^{2}\left(1-\nu^{2}\right)}{E}
$$

Figure 5.15 summarizes the J-integral calculations versus the crack size for the Cosserat problem, parametrized by $\alpha$. Figure 5.16 illustrates the normalized J-integral of the Cosserat elasticity, which is calculated as

$$
\frac{\left|J_{I}^{h}-J_{I}^{r e f}\right|}{J_{I}^{r e f}} \cdot 100 \%
$$

where $J_{I}^{h}$ is the J-integral for Cosserat problem, and $J_{I}^{r e f}$ is the J-integral for the classical elasticity problem (5.7).

It could be seen that the behaviour of the J-integral for the Cosserat elasticity is similar to that of the classical elasticity for the $0 \leq \alpha \leq 10^{3} \mathrm{~N} / \mathrm{mm}^{2}$. The values of the Cosserat J-integral for $10^{-1} \mathrm{~N} \leq \alpha \leq 10^{3} \mathrm{~N} / \mathrm{mm}^{2}$ are almost the same, with the smallest deviation from the classical J-integral of less than 5\%. For $\alpha=10^{4} \mathrm{~N} / \mathrm{mm}^{2}$, the values of the Cosserat J-integral is up to $40 \%$ higher than those of the classical one. The reason is that the parameter $\alpha$ is approaching the values of the couple stress theory. In that case, microrotations are no longer independent of the translations.

Based on the results of these studies, J-integral presented in [104] is valid for the 


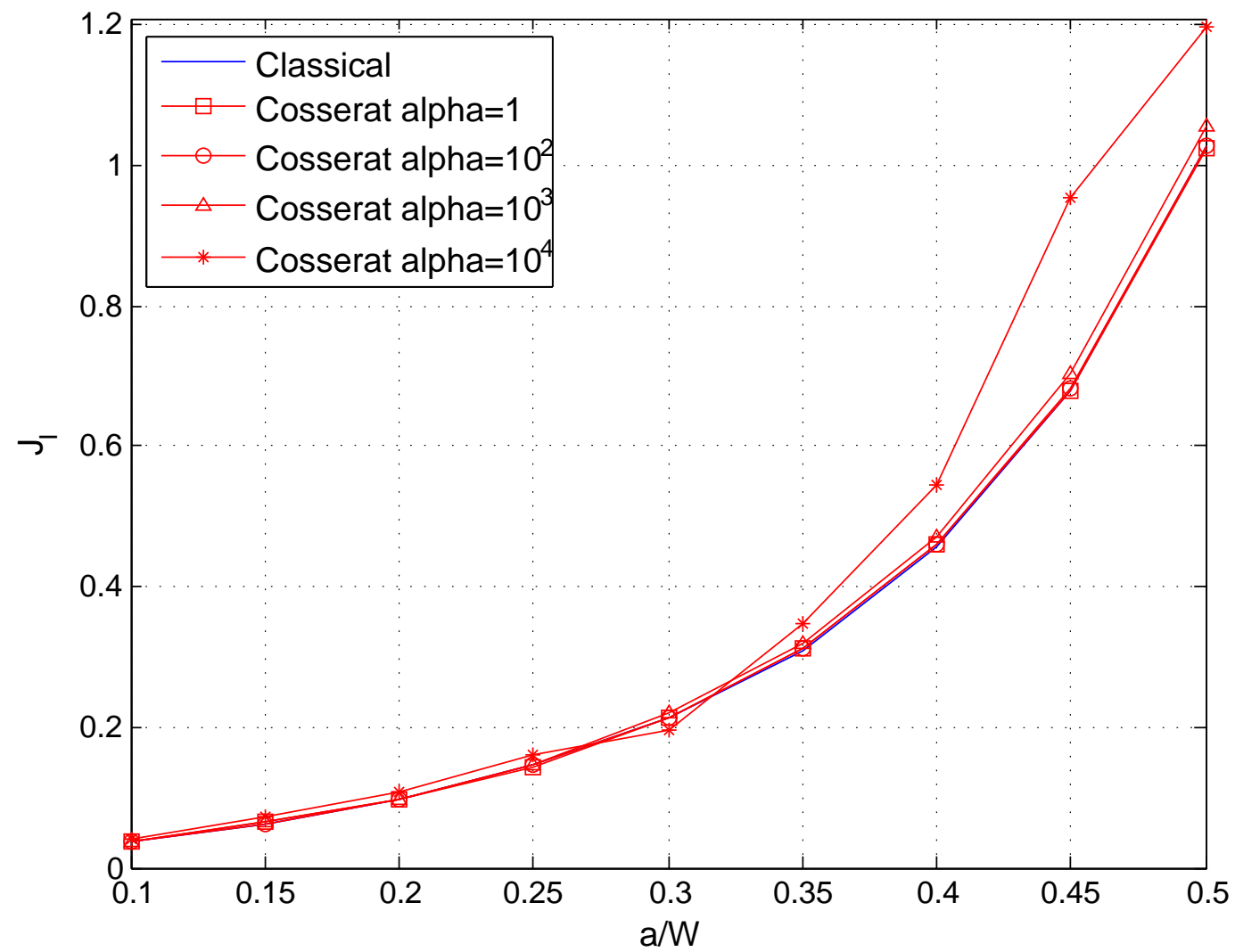

Figure 5.15: The J-integral versus the crack length.

calculations of the energy release rate in a Cosserat medium. In addition, the higher values of J-integral are obtained for the problems with the microrotations involved. 


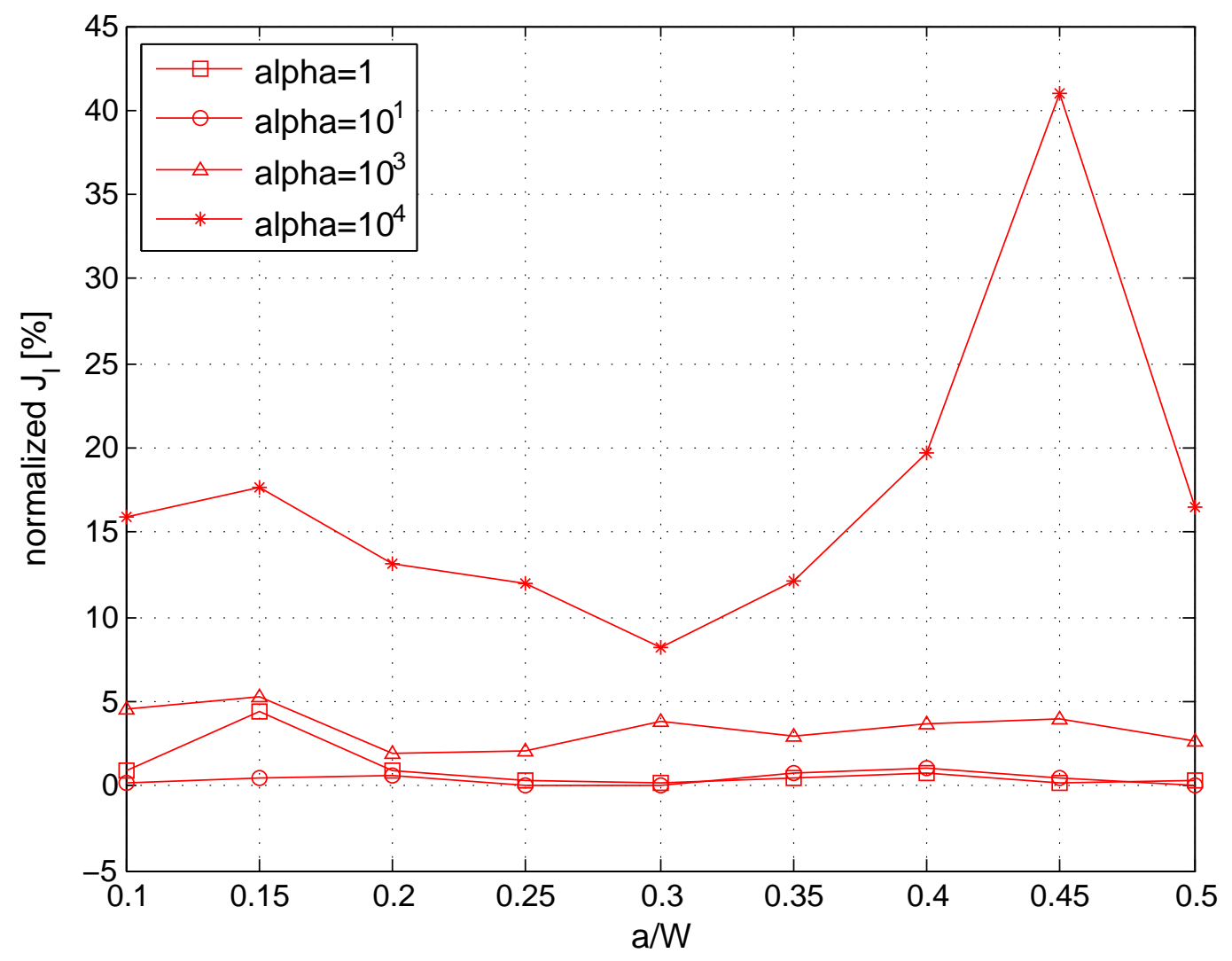

Figure 5.16: The normalized J-integral with respect to the crack length. 


\section{Chapter 6}

\section{Conclusions and Recommendations}

This chapter summarizes the main conclusions of the conducted research and makes recommendations for the future development of fracture modelling in a Cosserat medium.

\subsection{Concluding remarks}

Several objectives of the present research on crack opening simulation in Cosserat materials were identified at the beginning of the thesis. The main results and accomplishments of the research, addressing these objectives, are listed below:

- The primary goal of the research was to develop a robust and efficient method to simulate cracks in a Cosserat medium. The governing and discrete equations of the XFEM/Cosserat model were derived and presented in Chapter 4. The model was verified through the series of patch and convergence tests.

- The convergence study for the Finite Element Method and eXtended Finite Element Method were presented in Chapter 5. The optimal convergence rates for the XFEM solution of the Mode I and II edge crack problems were obtained. In addition, several cases depending on the tip enrichment zone (topological and geometrical) 
were investigated to get the optimal way to enrich the tip area for the further crack simulation.

- The calculation of J-integral was verified, allowing for reliance on the developed numerical technique. Particularly, under a wide range of input parameters, such as number of elements, the radius of the contour and the number of segments on the contour, the J-integral calculation exhibits consistent behaviour and predicts the same exact result.

- The J-integral for the Cosserat elastic medium was compared to the J-integral for the classical elastic medium, considering the example of the edge crack problem under

tension. It was found that the values of the J-integral of the Cosserat medium were up to $5 \%$ higher than those of the classical elastic medium when the micropolar material parameter $\alpha<10^{4} \mathrm{~N} / \mathrm{mm}^{2}$ was employed. For $\alpha \geq 10^{4} \mathrm{~N} / \mathrm{mm}^{2}$, the difference between the Cosserat and classical J-integral was up to $40 \%$ for the same problem. As mentioned above, parameter $\alpha$ governs the coupling level between translations and rotations with $\alpha=0$ corresponding to the classical elastic problem and $\alpha \rightarrow \infty$ corresponding to the couple stress problem.

\subsection{Recommendations for the future development}

The proposed XFEM model of an edge crack in a Cosserat medium can be extended in the several aspects described below.

First, further development of the proposed numerical XFEM/Cosserat model should address the improvement of the accuracy of the solution. It would be advisable to compare the convergence rates of the method considering various enrichment functions. The enrichment of the microrotation field is of special interest due to the lack of information about it in the literature.

Second, the presented model has the simple geometry of a straight line, and only the opening of the crack is considered; however, real cracks have more complicated geometry. 
The growth of the curved cracks, multiple and branching cracks in Cosserat media could be modelled.

Third, the initial XFEM/Cosserat crack model could be extended to the cohesive crack model. The presented model considers the fracture process zone to be small compared to the relevant dimensions of the specimen. This assumption neglects detailed description of what is happening in the fracture process zone. However, in reality, a small plastic zone will be formed around the crack tip. The cohesive crack model is able to transfer stress from one crack surface to another and describes the progressive fracture zone in full. The cohesive cracks in a Cosserat medium have not been studied or modelled in scientific community.

Considering the possible practical applications, the presented research may be a departure point to develop an accurate and realistic method for modelling fracture in geomechanics. In particular, these are problems of hydraulic fracture in materials such as shale rocks. The interest in development of the continuous model for the discontinuous rock masses is that, for the practical applications, a homogenized continuum model describes a large-scale response of the medium. Moreover, classical continuum theories cannot account for the elementary bending due to inter-block slip and may considerably overestimate the deformation [121]. For example, the discontinuous structure of shale rocks may be considered as the equivalent homogenized continuum using the Cosserat theory. This method takes into account the physical behaviour of material with a complex microstructure; it also considers the bending effect between the particles of material during the deformation by incorporating the intrinsic length scale into the governing equations of the system. The XFEM/Cosserat model along with the J-integral evaluation technique could be used to model full fracture process in the shale rock formations. 
APPENDICES 


\section{Appendix A}

\section{Matrix definitions}

The submatrices from the system (4.40) are

$$
\begin{aligned}
& \mathbf{K}_{11}=\int_{\Omega} \mathbf{B}^{\top} \mathbf{D}^{u} \mathbf{B} d \Omega, \\
& \mathbf{K}_{12}=\int_{\Omega} \mathbf{B}^{\top} \mathbf{D}^{u} \mathbf{T} \mathbf{N}^{\phi} d \Omega, \\
& \mathbf{K}_{13}=\int_{\Omega} \mathbf{B}^{\top} \mathbf{D}^{u} \overline{\mathbf{B}} d \Omega, \\
& \mathbf{K}_{14}=\int_{\Omega} \mathbf{B}^{\top} \mathbf{D}^{u} \mathbf{T} \overline{\mathbf{N}}^{\phi} d \Omega, \\
& \mathbf{K}_{15}=\int_{\Omega} \mathbf{B}^{\top} \mathbf{D}^{u} \widehat{\mathbf{B}} d \Omega, \\
& \mathbf{K}_{16}=\int_{\Omega} \mathbf{B}^{\top} \mathbf{D}^{u} \mathbf{T} \widehat{\mathbf{N}}^{\phi} d \Omega,
\end{aligned}
$$




$$
\begin{aligned}
& \mathbf{K}_{21}=-\int_{\Omega} \mathbf{N}^{\phi \top} \mathbf{T}^{\top} \mathbf{D}^{u} \mathbf{B} d \Omega, \\
& \mathbf{K}_{22}=\int_{\Omega}\left(\mathbf{B}^{\phi \top} \mathbf{D}^{\phi} \mathbf{B}^{\phi}-\mathbf{N}^{\phi \top} \mathbf{T}^{\top} \mathbf{D}^{u} \mathbf{T} \mathbf{N}^{\phi}\right) d \Omega, \\
& \mathbf{K}_{23}=-\int_{\Omega} \mathbf{N}^{\phi \top} \mathbf{T}^{\top} \mathbf{D}^{u} \overline{\mathbf{B}} d \Omega, \\
& \mathbf{K}_{24}=\int_{\Omega}\left(\mathbf{B}^{\phi \top} \mathbf{D}^{\phi} \overline{\mathbf{B}}^{\phi}-\mathbf{N}^{\phi \top} \mathbf{T}^{\top} \mathbf{D}^{u} \mathbf{T} \overline{\mathbf{N}}^{\phi}\right) d \Omega, \\
& \mathbf{K}_{25}=-\int_{\Omega} \mathbf{N}^{\phi \top} \mathbf{T}^{\top} \mathbf{D}^{u} \widehat{\mathbf{B}} d \Omega, \\
& \mathbf{K}_{26}=\int_{\Omega}\left(\mathbf{B}^{\phi \top} \mathbf{D}^{\phi} \widehat{\mathbf{B}}^{\phi}-\mathbf{N}^{\phi \top} \mathbf{T}^{\top} \mathbf{D}^{u} \mathbf{T} \widehat{\mathbf{N}}^{\phi}\right) d \Omega,
\end{aligned}
$$

$$
\begin{aligned}
& \mathbf{K}_{31}=\int_{\Omega} \overline{\mathbf{B}}^{\top} \mathbf{D}^{u} \mathbf{B} d \Omega, \\
& \mathbf{K}_{32}=\int_{\Omega} \overline{\mathbf{B}}^{\top} \mathbf{D}^{u} \mathbf{T} \mathbf{N}^{\phi} d \Omega, \\
& \mathbf{K}_{33}=\int_{\Omega} \overline{\mathbf{B}}^{\top} \mathbf{D}^{u} \overline{\mathbf{B}} d \Omega, \\
& \mathbf{K}_{34}=\int_{\Omega} \overline{\mathbf{B}}^{\top} \mathbf{D}^{u} \mathbf{T} \overline{\mathbf{N}}^{\phi} d \Omega, \\
& \mathbf{K}_{35}=\int_{\Omega} \overline{\mathbf{B}}^{\top} \mathbf{D}^{u} \widehat{\mathbf{B}} d \Omega, \\
& \mathbf{K}_{36}=\int_{\Omega} \overline{\mathbf{B}}^{\top} \mathbf{D}^{u} \mathbf{T} \widehat{\mathbf{N}^{\phi}} d \Omega,
\end{aligned}
$$




$$
\begin{aligned}
& \mathbf{K}_{41}=-\int_{\Omega} \overline{\mathbf{N}}^{\phi \top} \mathbf{T}^{\top} \mathbf{D}^{u} \mathbf{B} d \Omega, \\
& \mathbf{K}_{42}=\int_{\Omega}\left(\overline{\mathbf{B}}^{\phi \top} \mathbf{D}^{\phi} \mathbf{B}^{\phi}-\overline{\mathbf{N}}^{\phi \top} \mathbf{T}^{\top} \mathbf{D}^{u} \mathbf{T} \mathbf{N}^{\phi}\right) d \Omega, \\
& \mathbf{K}_{43}=-\int_{\Omega} \overline{\mathbf{N}}^{\phi \top} \mathbf{T}^{\top} \mathbf{D}^{u} \overline{\mathbf{B}} d \Omega, \\
& \mathbf{K}_{44}=\int_{\Omega}\left(\overline{\mathbf{B}}^{\phi \top} \mathbf{D}^{\phi} \overline{\mathbf{B}}^{\phi}-\overline{\mathbf{N}}^{\phi \top} \mathbf{T}^{\top} \mathbf{D}^{u} \mathbf{T} \overline{\mathbf{N}}^{\phi}\right) d \Omega, \\
& \mathbf{K}_{45}=-\int_{\Omega} \overline{\mathbf{N}}^{\phi \top} \mathbf{T}^{\top} \mathbf{D}^{u} \widehat{\mathbf{B}} d \Omega, \\
& \mathbf{K}_{46}=\int_{\Omega}\left(\overline{\mathbf{B}}^{\phi \top} \mathbf{D}^{\phi} \widehat{\mathbf{B}}^{\phi}-\overline{\mathbf{N}}^{\phi \top} \mathbf{T}^{\top} \mathbf{D}^{u} \mathbf{t} \widehat{\mathbf{N}}^{\phi}\right) d \Omega,
\end{aligned}
$$

$$
\begin{aligned}
\mathbf{K}_{51} & =\int_{\Omega} \widehat{\mathbf{B}}^{\top} \mathbf{D}^{u} \mathbf{B} d \Omega, \\
\mathbf{K}_{52} & =\int_{\Omega} \widehat{\mathbf{B}}^{\top} \mathbf{D}^{u} \mathbf{T} \mathbf{N}^{\phi} d \Omega, \\
\mathbf{K}_{53} & =\int_{\Omega} \widehat{\mathbf{B}}^{\top} \mathbf{D}^{u} \overline{\mathbf{B}} d \Omega, \\
\mathbf{K}_{54} & =\int_{\Omega} \widehat{\mathbf{B}}^{\top} \mathbf{D}^{u} \mathbf{T} \overline{\mathbf{N}}^{\phi} d \Omega, \\
\mathbf{K}_{55} & =\int_{\Omega} \widehat{\mathbf{B}}^{\top} \mathbf{D}^{u} \widehat{\mathbf{B}} d \Omega, \\
\mathbf{K}_{56} & =\int_{\Omega} \widehat{\mathbf{B}}^{\top} \mathbf{D}^{u} \mathbf{T} \widehat{\mathbf{N}}^{\phi} d \Omega,
\end{aligned}
$$




$$
\begin{aligned}
& \mathbf{K}_{61}=-\int_{\Omega} \widehat{\mathbf{N}}^{\phi \top} \mathbf{T}^{\top} \mathbf{D}^{u} \mathbf{B} d \Omega, \\
& \mathbf{K}_{62}=\int_{\Omega}\left(\widehat{\mathbf{B}}^{\phi \top} \mathbf{D}^{\phi} \mathbf{B}^{\phi}-\widehat{\mathbf{N}}^{\phi \top} \mathbf{T}^{\top} \mathbf{D}^{u} \mathbf{T} \mathbf{N}^{\phi}\right) d \Omega, \\
& \mathbf{K}_{63}=-\int_{\Omega} \widehat{\mathbf{N}}^{\phi \top} \mathbf{T D}^{u} \overline{\mathbf{B}} d \Omega, \\
& \mathbf{K}_{64}=\int_{\Omega}\left(\widehat{\mathbf{B}}^{\phi \top} \mathbf{D}^{\phi} \overline{\mathbf{B}}^{\phi}-\widehat{\mathbf{N}}^{\phi \top} \mathbf{T}^{\top} \mathbf{D}^{u} \mathbf{T} \overline{\mathbf{N}}^{\phi}\right) d \Omega, \\
& \mathbf{K}_{65}=-\int_{\Omega} \widehat{\mathbf{N}}^{\phi \top} \mathbf{T}^{\top} \mathbf{D}^{u} \widehat{\mathbf{B}} d \Omega, \\
& \mathbf{K}_{66}=\int_{\Omega}\left(\widehat{\mathbf{B}}^{\phi \top} \mathbf{D}^{\phi} \widehat{\mathbf{B}}^{\phi}-\widehat{\mathbf{N}}^{\phi \top} \mathbf{T}^{\top} \mathbf{D}^{u} \mathbf{T} \widehat{\mathbf{N}}^{\phi}\right) d \Omega, \\
& \mathbf{f}_{\mathbf{1}}=\int_{\Gamma_{t}} \mathbf{N}^{\top} \overline{\mathbf{t}} d \Gamma+\int_{\Omega} \mathbf{N}^{\top} \mathbf{b}^{\sigma} d \Omega, \\
& \mathbf{f}_{2}=\int_{\Gamma_{M}} \mathbf{N}^{\phi \top} \overline{\mathbf{M}} d \Gamma+\int_{\Omega} \mathbf{N}^{\phi \top} \mathbf{b}^{m} d \Omega, \\
& \mathbf{f}_{\mathbf{3}}=\int_{\Gamma_{t}} \overline{\mathbf{N}}^{\top} \overline{\mathbf{t}} d \Gamma+\int_{\Omega} \overline{\mathbf{N}}^{\top} \mathbf{b}^{\sigma} d \Omega, \\
& \mathbf{f}_{\mathbf{4}}=\int_{\Gamma_{M}} \overline{\mathbf{N}}^{\phi \top} \overline{\mathbf{M}} d \Gamma+\int_{\Omega} \overline{\mathbf{N}}^{\phi \top} \mathbf{b}^{m} d \Omega, \\
& \mathbf{f}_{\mathbf{5}}=\int_{\Gamma_{t}} \widehat{\mathbf{N}}^{\top} \overline{\mathbf{t}} d \Gamma+\int_{\Omega} \widehat{\mathbf{N}}^{\top} \mathbf{b}^{\sigma} d \Omega, \\
& \mathbf{f}_{\mathbf{6}}=\int_{\Gamma_{M}} \widehat{\mathbf{N}}^{\phi \top} \overline{\mathbf{M}} d \Gamma+\int_{\Omega} \widehat{\mathbf{N}}^{\phi \top} \mathbf{b}^{m} d \Omega,
\end{aligned}
$$

where 


$$
\boldsymbol{T}=\left[\begin{array}{c}
0 \\
0 \\
-1 \\
1
\end{array}\right]
$$

$$
\mathbf{B}_{I}=\left[\begin{array}{cc}
N_{I}(\mathbf{x})_{, x} & 0 \\
0 & N_{I}(\mathbf{x})_{, y} \\
0 & N_{I}(\mathbf{x})_{, x} \\
N_{I}(\mathbf{x})_{, y} & 0
\end{array}\right], \quad \forall I \in S_{H}
$$

$$
\mathbf{B}_{I}^{\phi}=\left[\begin{array}{c}
N_{I}(\mathbf{x})_{, x} \\
N_{I}(\mathbf{x})_{, y}
\end{array}\right], \quad \forall I \in S_{H},
$$

$$
\begin{gathered}
\overline{\mathbf{B}}_{J}=\left[\begin{array}{cc}
N_{J}(\mathbf{x})_{, x}\left(H(f(\mathbf{x}))-H\left(f\left(\mathbf{x}_{\mathbf{J}}\right)\right)\right) & 0 \\
0 & N_{J}(\mathbf{x})_{, y}\left(H(f(\mathbf{x}))-H\left(f\left(\mathbf{x}_{\mathbf{J}}\right)\right)\right) \\
0 & N_{J}(\mathbf{x})_{, x}\left(H(f(\mathbf{x}))-H\left(f\left(\mathbf{x}_{\mathbf{J}}\right)\right)\right)
\end{array}\right], \forall J \in S_{c r}, \\
N_{J}(\mathbf{x})_{, y}\left(H(f(\mathbf{x}))-H\left(f\left(\mathbf{x}_{\mathbf{J}}\right)\right)\right) \\
\overline{\mathbf{B}}_{J}^{\phi}=\left[\begin{array}{c}
N_{J}(\mathbf{x})_{, x}\left(H(f(\mathbf{x}))-H\left(f\left(\mathbf{x}_{\mathbf{J}}\right)\right)\right) \\
N_{J}(\mathbf{x})_{, y}\left(H(f(\mathbf{x}))-H\left(f\left(\mathbf{x}_{\mathbf{J}}\right)\right)\right.
\end{array}\right], \quad \forall J \in S_{c r},
\end{gathered}
$$




$$
\begin{gathered}
\widehat{\mathbf{B}}_{K}=\left[\begin{array}{cc}
N_{K}(\mathbf{x})_{, x} F_{m}(r, \theta)+N_{K}(\mathbf{x}) F_{m}(r, \theta)_{, x} & 0 \\
0 & N_{K}(\mathbf{x})_{, y} F_{m}(r, \theta)+N_{K}(\mathbf{x}) F_{m}(r, \theta)_{, y} \\
0 & N_{K}(\mathbf{x})_{, x} F_{m}(r, \theta)+N_{K}(\mathbf{x}) F_{m}(r, \theta)_{, x} \\
N_{K}(\mathbf{x})_{, y} F_{m}(r, \theta)+N_{K}(\mathbf{x}) F_{m}(r, \theta)_{, y} & 0
\end{array}\right], \forall K \in S_{t i p}, \\
\widehat{\mathbf{B}}_{K}^{\phi}=\left[\begin{array}{c}
N_{K}(\mathbf{x})_{, x} F^{\phi}(r, \theta)+N_{K}(\mathbf{x}) F^{\phi}(r, \theta)_{, x} \\
N_{K}(\mathbf{x})_{, y} F^{\phi}(r, \theta)+N_{K}(\mathbf{x}) F^{\phi}(r, \theta)_{, y}
\end{array}\right], \quad \forall K \in S_{t i p .}
\end{gathered}
$$




\section{References}

[1] A.L. Cauchy. Cours d'analyse de l'École Royale Polytechnique. Imprimerie royale, 1821.

[2] C.L.M.H. Navier. Résumé des leçons données à l'École royale des ponts et chaussées sur l'application de la mécanique à l'établissement des constructions et des machines. Didot, 1826.

[3] C. Dai, H. Mühlhaus, J. Meek, and M.D. Fama. Modelling of blocky rock masses using the cosserat method. In International Journal of Rock Mechanics and Mining Sciences $\& 6$ Geomechanics Abstracts, volume 33, pages 425-432. Pergamon, 1996.

[4] A.R. Khoei and K. Karimi. An enriched-fem model for simulation of localization phenomenon in cosserat continuum theory. Computational Materials Science, 44(2):733$749,2008$.

[5] A.R. Khoei, S. Yadegari, and S.O.R. Biabanaki. 3d finite element modeling of shear band localization via the micro-polar cosserat continuum theory. Computational Materials Science, 49(4):720-733, 2010.

[6] A. Riahi and J.H. Curran. Full 3d finite element cosserat formulation with application in layered structures. Applied Mathematical Modelling, 33(8):3450-3464, 2009.

[7] M. Gei, M. Rovati, and D. Veber. Effect of internal length scale on optimal topologies for cosserat continua. In IUTAM Symposium on Topological Design Optimization of Structures, Machines and Materials, pages 157-166. Springer, 2006. 
[8] A.C. Eringen. Linear theory of micropolar elasticity. Journal of Mathematics and Mechanics, 15:909-923, 1966.

[9] E. Cosserat, F. Cosserat, M. Brocato, and K. Chatzis. Théorie des corps déformables. A. Hermann, 1909.

[10] W. Nowacki. Theory of micropolar elasticity. Springer, 1972.

[11] J.F.C. Yang and R.S. Lakes. Experimental study of micropolar and couple stress elasticity in compact bone in bending. Journal of biomechanics, 15(2):91-98, 1982.

[12] R.S. Lakes. Size effects and micromechanics of a porous solid. Journal of materials science, 18(9):2572-2580, 1983.

[13] N.P. Kruyt. Statics and kinematics of discrete cosserat-type granular materials. International journal of solids and structures, 40(3):511-534, 2003.

[14] I. Münch, P. Neff, and W. Wagner. Transversely isotropic material: nonlinear cosserat versus classical approach. Continuum Mechanics and Thermodynamics, 23(1):27-34, 2011.

[15] J. Yang and G. Jiang. Experimental study on properties of pervious concrete pavement materials. Cement and Concrete Research, 33(3):381-386, 2003.

[16] J. Fatemi, F. Van Keulen, and PR Onck. Generalized continuum theories: Application to stress analysis in bone. Meccanica, 37(4):385-396, 2002.

[17] S.P. Timoshenko and J.N. Goodier. Theory of Elasticity. McGraw-Hill, 1970.

[18] W. Voigt. Theoretische studien fiber die elastizitatsverhiltnisse der kristalle (theoretical studies on the elasticity relationships of crystals). Abh. Gesch. Wissenschaften, 34, 1887.

[19] P. Duhem. Physique et métaphysique. Polleunis, 1893.

[20] R.A. Toupin. Elastic materials with couple-stresses. Archive for Rational Mechanics and Analysis, 11(1):385-414, 1962. 
[21] C. Truesdell and R. Toupin. The classical field theories. Springer, 1960.

[22] V.D. Kupradze, T.G. Gelelia, M.O. Basheleïshvili, and T.V. Burchuladze. Threedimensional problems in the mathematical theory of elasticity and thermoelasticity: Classical and micropolar theory. statics, harmonic oscillations, dynamics. fundamentals and solution methods//(russian book). Moscow, Izdatel'stvo Nauka, 1976. 664, 1976.

[23] W. Günther. Zur statik und kinematik des cosseratschen kontinuums. 1958.

[24] H. Schaefer. Das cosserat kontinuum. ZAMM-Journal of Applied Mathematics and Mechanics/Zeitschrift für Angewandte Mathematik und Mechanik, 47(8):485-498, 1967.

[25] H. Schaefer. Analysis der motorfelder im cosserat-kontinuum. ZAMM-Journal of Applied Mathematics and Mechanics/Zeitschrift für Angewandte Mathematik und Mechanik, 47(5):319-328, 1967.

[26] E.L. Aero and E.V. Kuvshinski. Continuum theory of asymmetric elasticity. equilibrium of an isotropic body. Solid State Physics, 6(9):2689-2699, 1964.

[27] V.A. Palmov. Fundamental equations of the theory of nonsymmetric elasticity. Prikl. Math. Mech., 1964.

[28] A.C. Eringen and E.S. Suhubi. Nonlinear theory of simple micro-elastic solids i. International Journal of Engineering Science, 2(2):189-203, 1964.

[29] R. Lakes. On the torsional properties of single osteons. Journal of biomechanics, 28(11):1409-1409, 1995.

[30] R.S. Lakes. Experimental methods for study of cosserat elastic solids and other generalized elastic continua. Continuum models for materials with microstructure, pages $1-25,1995$.

[31] R.S. Lakes and J.F.C. Yang. Micropolar elasticity in bone: rotation modulus $\kappa$. In Proceedings 18th Midwest Mechanics Conference, Iowa City, Developments in Mechanics, volume 12, pages 239-242, 1983. 
[32] S. Nakamura and R.S. Lakes. Finite element analysis of stress concentration around a blunt crack in a cosserat elastic solid. Computer methods in applied mechanics and engineering, 66(3):257-266, 1988.

[33] P.N. Kaloni and T. Ariman. Stress concentration effects in micropolar elasticity. Zeitschrift für Angewandte Mathematik und Physik (ZAMP), 18(1):136-141, 1967.

[34] B.S. Kim. Stress distribution around an elliptic hole in an infinite micropolar elastic plate. Technical report, DTIC Document, 1973.

[35] R.S. Lakes, S. Nakamura, J.C. Behiri, and W. Bonfield. Fracture mechanics of bone with short cracks. Journal of biomechanics, 23(10):967-975, 1990.

[36] B. Singh. Continuum characterization of jointed rock masses: Part ithe constitutive equations. 10(4):311-335, 1973.

[37] B. Singh. Continuum characterization of jointed rock masses: Part iisignificance of low shear modulus. 10(4):337-349, 1973.

[38] H.B. Mühlhaus. Continuum models for materials with microstructure. Wiley, 1995.

[39] H.B. Mühlhaus and I. Vardoulakis. The thickness of shear bands in granular materials. Geotechnique, 37(3):271-283, 1987.

[40] C. Dai, H. Muhlhaus, M. Duncan Fama, and J. Meek. Finite element analysis of cosserat theory for layered rock masses. Computers and Geotechnics, 15(3):145-162, 1993.

[41] H. Yang and K. Wang. Combined identification of constitutive parameters and insitu stresses for an elastic cosserat model of an excavation in a layered rock mass. International Journal of Rock Mechanics and Mining Sciences, 46(3):659-664, 2009.

[42] J. Sulem, I. Vardoulakis, and E. Papamichos. Microstructure and scale effect in granular rocks. Continuum Models for Materials with Microstructure, H.-B. Mühlhaus, Ed.(J. Wiley 83 Sons, Chichester, 1995) pp, pages 200-237, 1995. 
[43] E. Papamichos. Continua with microstructure: Cosserat theory. European Journal of Environmental and Civil Engineering, 14(8-9):1011-1029, 2010.

[44] P. Neff. Cosserat theory, 2010.

[45] AC Eringen. Microcontinuum field theories. i. foundations and solids. 1999.

[46] R.S. Lakes. A pathological situation in micropolar elasticity. Journal of applied mechanics, 52(1):234-235, 1985.

[47] R.D. Gauthier and W.E. Jahsman. A quest for micropolar elastic constants. In Journal of Applied Mechanics, number 42, pages 369-374, 1975.

[48] J. Ozbolt and Z.P. Bazant. Numerical smeared fracture analysis: Nonlocal microcrack interaction approach. International Journal for Numerical Methods in Engineering, 39(4):635-662, 1996.

[49] K. Willam, E. Pramono, and S. Sture. Fundamental issues of smeared crack models. In Proc. SEM-RILEM International Conference on Fracture of Concrete and Rock, pages 192-207. SEM, Bethel, 1987.

[50] M. Prasad and C.S. Krishnamoorthy. Computational model for discrete crack growth in plain and reinforced concrete. Computer methods in applied mechanics and engineering, 191(25-26):2699-2725, 2002.

[51] S. Mohammadi. Extended finite element method. Chichester, West Sussex, England: John Wiley \& Sons, 2008.

[52] M. Chaudonneret. On the discontinuity of the stress vector in the boundary integral equation method for elastic analysis. Recent advances in boundary element methods.(A 79-38851 16-64) London, Pentech Press, Ltd., pages 185-194, 1978.

[53] S. Potapenko and E. Shmoylova. Solvability of weak solutions to anti-plane cosserat elasticity by means of boundary integral equations. Mathematics and Mechanics of Solids, 15(2):209-228, 2010. 
[54] E. Shmoylova, S. Potapenko, and L. Rothenburg. Boundary element analysis of stress distribution around a crack in plane micropolar elasticity. International journal of engineering science, 45(2):199-209, 2007.

[55] A. Portela, M.H. Aliabadi, and D.P. Rooke. The dual boundary element method: effective implementation for crack problems. International Journal for Numerical Methods in Engineering, 33(6):1269-1287, 1992.

[56] T. Belytschko and T. Black. Elastic crack growth in finite elements with minimal remeshing. International Journal for Numerical Methods in Engineering, 45:601-620, 1999.

[57] T. Belytschko and T. Black. Elastic crack growth in finite elements with minimal remeshing. International Journal for Numerical Methods in Engineering, 45(5):601620, 1999 .

[58] P.A. Cundall and O.D.L. Strack. A discrete numerical model for granular assemblies. Geotechnique, 29(1):47-65, 1979.

[59] T. Belytschko, Y. Krongauz, D. Organ, M. Fleming, and P. Krysl. Meshless methods: an overview and recent developments. Computer methods in applied mechanics and engineering, 139(1-4):3-47, 1996.

[60] T. Belytschko, N. Moës, S. Usui, and C. Parimi. Arbitrary discontinuities in finite elements. International Journal for Numerical Methods in Engineering, 50(4):9931013, 2001.

[61] T. Belytschko and R. Gracie. On xfem applications to dislocations and interfaces. International Journal of Plasticity, 23(10-11):1721-1738, 2007.

[62] N. Sukumar, D.L. Chopp, N. Moës, and T. Belytschko. Modeling holes and inclusions by level sets in the extended finite-element method. Computer Methods in Applied Mechanics and Engineering, 190:6183-6200, 2001. 
[63] R. Gracie, G. Ventura, and T. Belytschko. A new fast method for dislocations based on interior discontinuities. International Journal for Numerical Methods in Engineering, 69:423-441, 2007.

[64] R. Gracie and T. Belytschko. Concurrently coupled atomistic and xfem models for dislocations and cracks. International Journal for Numerical Methods in Engineering, 78(3):354-378, 2009.

[65] J. Oswald, R. Gracie, R. Khare, and T. Belytschko. An extended finite element method for dislocations in complex geometries: thin films and nanotubes. Computer Methods in Applied Mechanics and Engineering, 198(21-26):1872-1886, 2009.

[66] R. Gracie, J. Oswald, and T. Belytschko. On a new extended finite element method for dislocations: core enrichments. Journal of the Mechanics and Physics of Solids, 56:200-214, 2008.

[67] Hansbo A. and Hansbo P. A finite element method for the simulation of strong and weak discontinuities in solid mechanics. Computer Methods in Applied Mechanics and Engineering, 193:3523-3540, 2004.

[68] T. Belytschko, R. Gracie, and G. Ventura. A review of extended/generalized finite element methods for material modeling. Modelling and Simulation in Materials Science and Engineering, 17:043001, 2009.

[69] N. Moës, J. Dolbow, and T. Belytschko. A finite element method for crack growth without remeshing. International Journal for Numerical Methods in Engineering, 46:131-150, 1999.

[70] J.M. Melenk and I. Babuška. The partition of unity finite element method: Basic theory and applications. Computer Methods in Applied Mechanics and Engineering, 139:290-314, 1996.

[71] J.E. Dolbow. An extended finite element method with discontinuous enrichment for applied mechanics. Northwestern University, 1999. 
[72] J. Dolbow, N. Moës, and T. Belytschko. Discontinuous enrichment in finite elements with a partition of unity method. Finite Elements in Analysis and Design, 36(34):235-260.

[73] J. Dolbow, N. Moës, and T. Belytschko. Modeling fracture in mindlin-reissner plates with the extended finite element method. International Journal of Solids and Structures, 37(48):7161-7183, 2000.

[74] N. Sukumar, N. Moës, B. Moran, and T Belytschko. Extended finite element method for three-dimensional crack modelling. International Journal for Numerical Methods in Engineering, 48:1549-1570, 2000.

[75] M. Stolarska, D. L. Chopp, N. Moës, and T. Belytschko. Modelling crack growth by level sets in the extended finite element method. International Journal for Numerical Methods in Engineering, 51(8):943-960, 2001.

[76] C. Daux, N. Moës, J. Dolbow, N. Sukumar, and T. Belytschko. Arbitrary branched and intersecting cracks with the extended finite element method. International Journal for Numerical Methods in Engineering, 48(12):1741-1760, 2000.

[77] E. Budyn, G. Zi, N. Moës, and T. Belytschko. A method for multiple crack growth in brittle materials without remeshing. International journal for numerical methods in engineering, 61(10):1741-1770, 2004.

[78] N. Sukumar and T. Belytschko. Arbitrary branched and intersecting cracks with the extended finite element method. International Journal for Numerical Methods in Engineering, 48:1741-1760, 2000.

[79] F.L. Stazi, E. Budyn, J Chessa, and T. Belytschko. An extended finite element method with higher-order elements for curved cracks. Computational Mechanics, 31:38-48, 2003.

[80] N. Moës and T. Belytschko. Extended finite element method for cohesive crack growth. Engineering Fracture Mechanics, 69(7):813-833, 2002. 
[81] J. Dolbow, N. Moës, and T. Belytschko. An extended finite element method for modeling crack growth with frictional contact. Computer Methods in Applied Mechanics and Engineering, 190(51-52):6825-6846, 2001.

[82] A.R. Khoei and M. Nikbakht. Contact friction modeling with the extended finite element method (x-fem). Journal of materials processing technology, 177(1):58-62, 2006.

[83] T. Elguedj, A. Gravouil, and A. Combescure. Appropriate extended functions for x-fem simulation of plastic fracture mechanics. Computer Methods in Applied Mechanics and Engineering, 195(7-8):501-515, 2006.

[84] G. Legrain, E. Verron, and N. Moës. Numerical simulation of large strain fracture problems using $\mathrm{x}$-fem. In Constitutve models for rubber-proceedings, volume 4 , page 59. Balkema, 2005.

[85] E. Budyn, T. Hoc, and J. Jonvaux. Fracture strength assessment and aging signs detection in human cortical bone using an x-fem multiple scale approach. Computational Mechanics, 42(4):579-591, 2008.

[86] É. Budyn and T. Hoc. Analysis of micro fracture in human haversian cortical bone under transverse tension using extended physical imaging. International Journal for Numerical Methods in Engineering, 82(8):940-965, 2010.

[87] X.Y. Liu, Q.Z. Xiao, and B.L. Karihaloo. XFEM for direct evaluation of mixed mode SIFs in homogeneous and bi-materials. International Journal for Numerical Methods in Engineering, 59:1103-1118, 2004.

[88] A. Zamani, R. Gracie, and M.R. Eslami. Higher order tip enrichment of extended finite element method in thermoelasticity. Computational Mechanics, pages 1-16, 2010 .

[89] E.E. Gdoutos. Fracture mechanics: an introduction, volume 123. Kluwer Academic Pub, 2005.

[90] A.A. Griffith. The theory of rupture. 1997. 
[91] G.P. Cherepanov. The propagation of cracks in a continuous medium. Journal of Applied Mathematics and Mechanics, 31(3):503-512, 1967.

[92] J.R. Rice. A path independent integral and the approximate analysis of strain concentration by notches and cracks. Technical report, DTIC Document, 1967.

[93] T. Anderson. Fracture mechanics: fundamentals and Applications. CRC Press, 1995.

[94] G.R. Irwin. Analysis of stresses and strains near the end of a crack traversing a plate. J. appl. Mech., 1957.

[95] Z.P. Bažant and J. Planas. Fracture and size effect in concrete and other quasibrittle materials. CRC, 1998.

[96] H.B. Mühlhaus and E. Pasternak. Path independent integrals for cosserat continua and application to crack problems. International journal of fracture, 113(4):21-26, 2002.

[97] E. Pasternak, A.V. Dyskin, and H.B. Mühlhaus. Cracks of higher modes in cosserat continua. International journal of fracture, 140(1):189-199, 2006.

[98] E. Diegele, R. ElsÄßer, and C. Tsakmakis. Linear micropolar elastic crack-tip fields under mixed mode loading conditions. International journal of fracture, 129(4):309339, 2004.

[99] G. Exadaktylos, I. Vardoulakis, and E. Aifantis. Cracks in gradient elastic bodies with surface energy. International Journal of Fracture, 79(2):107-119, 1996.

[100] Y. Huang, L. Zhang, TF Guo, and K.C. Hwang. Mixed mode near-tip fields for cracks in materials with strain-gradient effects. Journal of the Mechanics and Physics of Solids, 45(3):439-465, 1997.

[101] L. Zhang, Y. Huang, JY Chen, and KC Hwang. The mode iii full-field solution in elastic materials with strain gradient effects. International Journal of Fracture, 92(4):325-348, 1998. 
[102] C. Atkinson and F.G. Leppington. The effect of couple stresses on the tip of a crack. International Journal of Solids and Structures, 13(11):1103-1122, 1977.

[103] E. Sternberg and R. Muki. The effect of couple-stresses on the stress concentration around a crack. International Journal of Solids and Structures, 3(1):69-95, 1967.

[104] V.A. Lubarda and X. Markenscoff. On conservation integrals in micropolar elasticity. Philosophical Magazine, 83(11):1365-1377, 2003.

[105] C. Atkinson and F.G. Leppington. Some calculations of the energy-release rate $\mathrm{g}$ for cracks in micropolar and couple-stress elastic media. International Journal of Fracture, 10(4):599-602, 1974.

[106] J.D. Eshelby. The elastic energy-momentum tensor. Journal of Elasticity, 5(3):321$335,1975$.

[107] E. Providas and M.A. Kattis. Finite element method in plane cosserat elasticity. CS, 80(27-30):2059-2069, 2002.

[108] D.U. Yang and F.Y. Huang. Analysis of poissons ratio for a micropolar elastic rectangular plate using the finite element method. Engineering Computations, 18(7):10121030, 2001.

[109] E. Papamichos, I. Vardoulakis, and H.B. Mühlhaus. Buckling of layered elastic media: A cosserat-continuum approach and its validation. International Journal for Numerical and Analytical Methods in Geomechanics, 14(7):473-498, 1990.

[110] I. Vardoulakis and E.C. Aifantis. On the role of microstructure in the behavior of soils: effects of higher order gradients and internal inertia. Mechanics of materials, 18(2):151-158, 1994.

[111] D.P. Adhikary and A.V. Dyskin. A cosserat continuum model for layered materials. Computers and Geotechnics, 20(1):15-45, 1997.

[112] DP Adhikary and H. Guo. An orthotropic cosserat elasto-plastic model for layered rocks. Rock mechanics and rock engineering, 35(3):161-170, 2002. 
[113] A.R. Khoei, M. Anahid, and K. Shahim. An extended arbitrary lagrangian-eulerian finite element method for large deformation of solid mechanics. Finite Elements in Analysis and Design, 44(6-7):401-416, 2008.

[114] J. Fish and T. Belytschko. Front Matter. Wiley Online Library, 2007.

[115] J.N. Reddy. An introduction to the finite element method, volume 2. McGraw-Hill New York, 1993.

[116] T.P. Fries. A corrected xfem approximation without problems in blending elements. International Journal for Numerical Methods in Engineering, 75(5):503-532, 2008.

[117] G. Ventura, R. Gracie, and T. Belytschko. Fast integration and weight function blending in the extended finite element method. International Journal for Numerical Methods in Engineering, 77(1):1-29, 2009.

[118] E. Béchet, H. Minnebo, N. Moës, and B. Burgardt. Improved implementation and robustness study of the X-FEM for stress analysis around cracks. International Journal for Numerical Methods in Engineering, 64(8):1033-1056, 2005.

[119] P. Laborde, J. Pommier, Y. Renard, and M. Salaun. High-order extended finite element method for cracked domains. International Journal for Numerical Methods in Engineering, 64(3):354-381, 2005.

[120] H. Tada, P.C. Paris, G.R. Irwin, and H. Tada. The stress analysis of cracks handbook, volume 130. ASME press New York, 2000.

[121] I. Stefanou and J. Sulem. Continuum modeling of discontinuous rock structures. European Journal of Environmental and Civil Engineering, 14(8-9):1199-1217, 2010. 